LAUR- $98-2413$

\title{
Magnetized Target Fusion A Proof-of-Principle Research Proposal
}

Submitted to the Office of Fusion Energy Sciences

$$
\text { May 19, } 1998
$$

K. F. Schoenberg and R. E. Siemon

\section{Thin imploding liner}

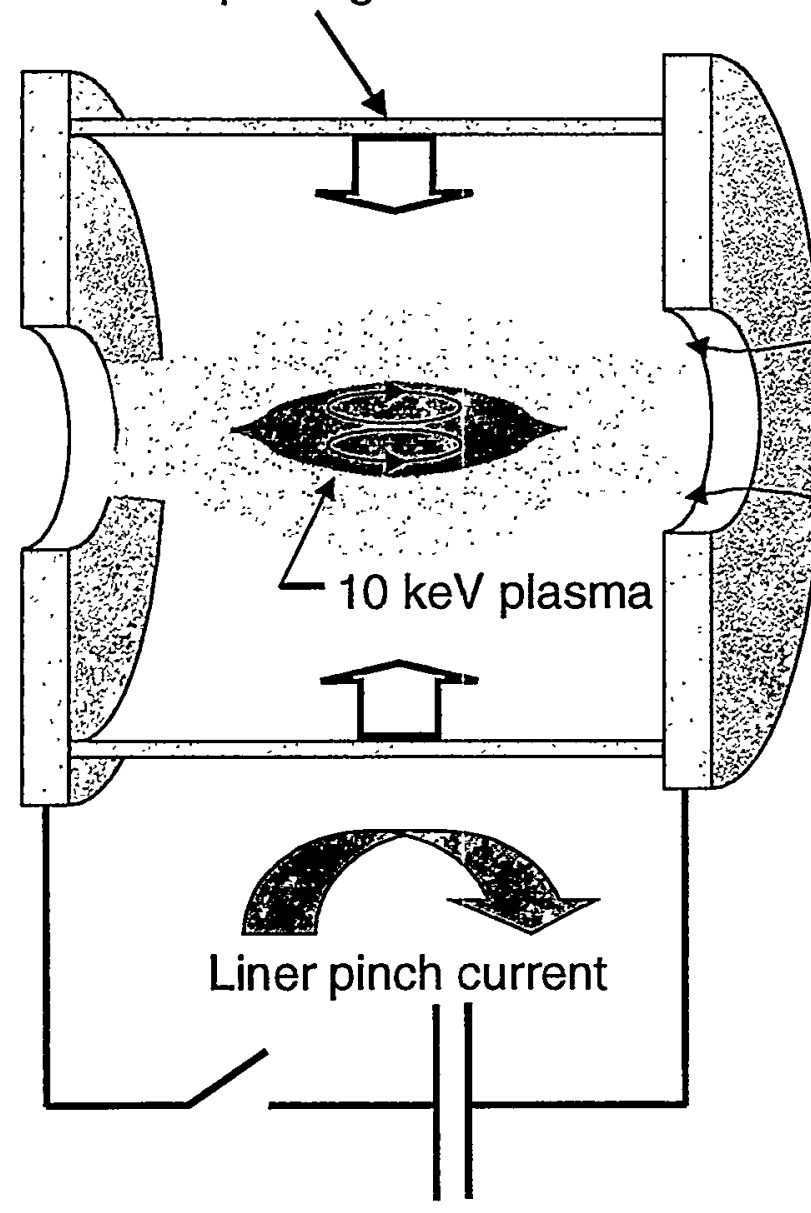

Plasma preheater and injector

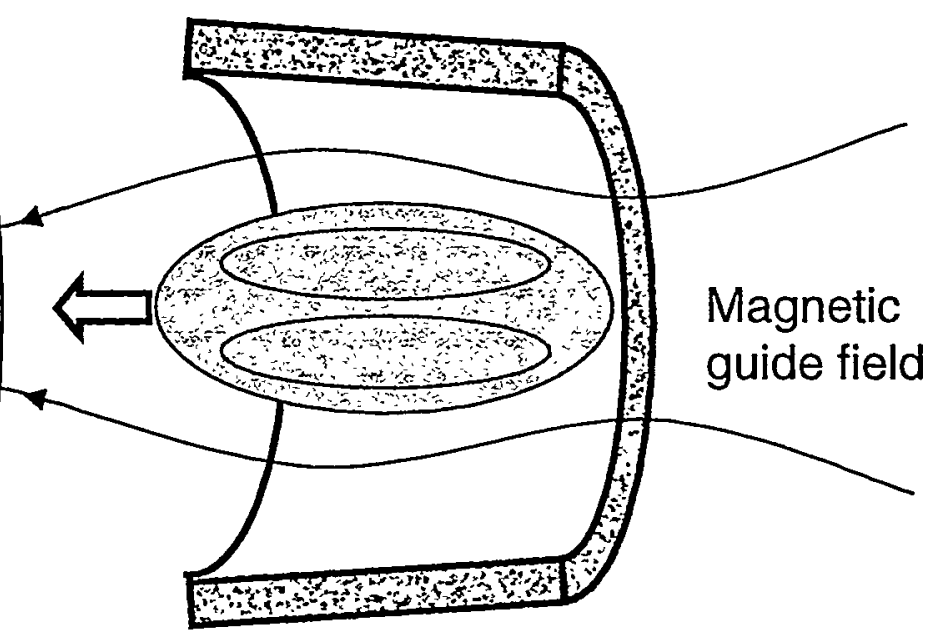

Los Alamos National Laboratory 


\section{DISCLAIMER}

This report was prepared as an account of work sponsored by an agency of the United States Government. Neither the United States Government nor any agency thereof, nor any of their employees, make any warranty, express or implied, or assumes any legal liability or responsibility for the accuracy, completeness, or usefulness of any information, apparatus, product, or process disclosed, or represents that its use would not infringe privately owned rights. Reference herein to any specific commercial product, process, or service by trade name, trademark, manufacturer, or otherwise does not necessarily constitute or imply its, endorsement, recommendation, or favoring by the United States Government or any agency thereof. The views and opinions of authors expressed herein do not necessarily state or reflect those of the United States Government or any agency thereof. 


\section{DISCLAIMER}

Portions of this document may be illegible in electronic image products. Images are produced from the best available original document. 


\title{
Magnetized Target Fusion A Proof-of-Principle Research Proposal
}

\author{
Submitted to the Omce of Fusion Energy Sciences
}

May 19, 1998

PoP Program Leaders and Editors:

K. F. Schoenberg and R. E. Siemon

PoP Participants and Contributors:

R. J. Bartlett, R. R. Bartsch, C. A. Ekdahl, R. J. Faehl, J.Finn, R. A. Gerwin, R. Kirkpatrick,

I. R. Lindemuth, R. W. Moses, R. E. Reinovsky, K. F, Schoenberg, P. T. Sheehey,

J. S. Shlachter, M. G. Tuszewski, G. A. Wurden, and F. J. Wysocki, Los Alamos National

Laboratory; J. Degnan, G. F. Kiuttu, E. L. Ruden, and P. Turchi, Air Force Research Laboratory;

P. Parks, General Atomics; J. H. Hammer, R. Moir, and D. Ryutov, Lawrence Livermore

National Laboratory; Y.C.F. Thio, Massey University, New Zealand; R. D. Milroy,

U. Washington; L. Green, Westinghouse Science and Technology Center.

Disclaimer: The budget estimates in this proposal are unofficial in the sense that they have nor been reviewed and approved by the budgeting authorities of the various institutions involved. However, in every case the numbers have been checked by principal investigators and appropriate line managers of the respective institutions, so they should serve as useful estimates for planning purposes.

Revision 1: May 21, 1998 


\section{TABLE OF CONTENTS}

Section

Page

EXECUTTVE SUMMARY

iii

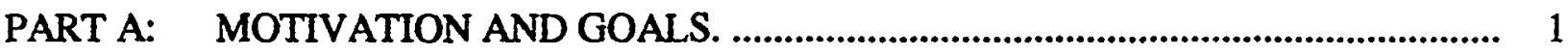

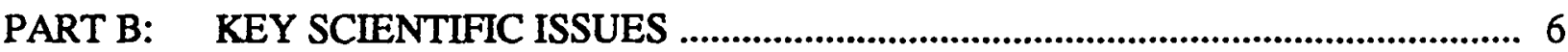

PART C: RESEARCH PROGRAM ................................................................... 16

PART D: POTENTIAL FOR PRACTICAL FUSION POWER ................................. 31

PART E: $\quad$ COMMUNITY R\&D PLAN .............................................................. 34

PART F: $\quad$ LITERATURE CITED ......................................................................... 35

APPENDIX A: Why Magnetized Target Fusion Offers A Low-Cost Development Path for Fusion Energy ............................................................................... 1

APPENDIX B: $\quad$ Other MTF Target Plasmas ...................................................................... 20

APPENDIX C: Theory of Wall-Plasma Interactions ......................................................24

APPENDIX D: Proposing Team Biographies....................................................................34 


\section{Executive Summary:}

We propose herein a three-year, $\$ 6.6$ million per year, proof-of-principle (PoP) program to establish the scientific basis of Magnetized Target Fusion (MTF) as a faster and cheaper approach to fusion energy. To explore this truly different fusion concept, we will take advantage of compact toroid (CT) research developed by the magnetic fusion energy program (MFE) in the past 20 years. The CT plasma is the target, which we will implode using well-established liner technology developed by DOE and DOD defense programs research in recent years. The magnetic topology of the CT should provide enough thermal energy confinement to allow compressional heating of the plasma to fusion-relevant conditions. Fusion energy will be generated in a micro-second pulse during which pressure (plasma and magnetic) is inertially confined by the imploding liner wall. This specific CT approach to small-size, high-density fusion (by MFE standards) is intended to prove the principle of MTF by achieving significant

performance ( $\mathrm{m} \tau>10^{13} \mathrm{~s}-\mathrm{cm}^{-3}, \mathrm{~T}-5 \mathrm{keV}$ ) in just a few years at modest cost using available pulsed-power facilities. Success in understanding will require both experiments and large-scale numerical computation.

The density regime and time scale of MTF is intermediate between MFE and inertial-confinement fusion (ICF). Three technical considerations explain why the regime is important. First, fusion reactivity scales as density squared, which can be increased by many orders of magnitude over conventional MFE. Second, all characteristic plasma scale-lengths decrease with density. Hence, system size is naturally reduced at a high density. Third, magnetic insulation greatly reduces the required power and precision to compressionally-heat plasma to fusion-relevant conditions compared with ICF, and brings the pulsedpower requirements within reach of existing facilities. Thus, we conclude the intermediate density regime holds promise as a new low-cost avenue to fusion energy. The future path for engineering development of MTF as an economic power source is less well defined than for the more mature approaches of MFE and ICF. However, a number of possibilities are being discussed, and this research program will include scoping studies to identify the most promising approaches.

The research of this proposal is focused on several critical issues for application of a CT target plasma to MTF:

- formation at required density $\left(-10^{17} \mathrm{~cm}^{-3}\right)$ and temperature $(-300 \mathrm{eV})$,

- stability during formation and compression,

- energy confinement adequate for fast liner compressional heating,

- plasma impurity content and the general consequences of high-energy-density plasma-wall interactions

In pursuit of these research goals, Los Alamos will lead a multi-institutional team formed to elucidate the physics underlying these technical challenges. This team of collaborators is critical to the proposal and the assessment of MTF as a fusion approach. The total budget to support this research across all institutions is $\$ 6.6 \mathrm{M} /$ year for three years.

Our principal target plasma candidate is the field-reversed-configuration (FRC). The FRC offers the promise of robust, closed flux surfaces, capable of maintaining their topology during compression. Past experiments have demonstrated that an FRC can be translated and trapped in a liner implosion geometry. 
Most importantly, formation of an FRC using high-yoltage theta-pinch technology is well established, and appears likely to extrapolate to the required target requirements. The existing database for energy confinement scaling suggests the FRC will work well in an MTF application. The cylindrical geometry of the FRC allows end-on diagnostic access even during energetic liner implosions. All together, the physics base developed by over twenty years of FRC research gives confidence that FRC targets have a reasonable chance of being compressed to fusion-relevant conditions.

High-speed liners, composed principally of aluminum, are the best candidates to compress the FRC target plasma. Defense-program-developed imploding liners have demonstrated the speed and convergence required for MTF PoP experiments. This knowledge base, coupled with well-benchmarked analytic and computational models of liner physics, provide confidence that liner performance suitable for an MTF PoP program is in hand. Furthermore, PoP liner-plasma compression experiments can be executed on existing pulsed-power facilities.

As detailed in the following proposal, the first two years of research will focus on creating an FRC target plasma suitable for compression. Here, we will form dense, elongated FRCs that are within known operational boundaries of dimensionless parameters, where energy confinement has been observed to be optimum. In parallel, a liner to compress the elongated FRC will be designed and tested without a plasma. Based on the success of the first two years of research, the third year will focus on integrated liner-onplasma compression experiments. This research will give improved understanding of FRC formation, stability, and transport at high density and temperature. Performance expectations encompass temperature, density, and $\mathrm{nt}$ values of $5 \mathrm{keV}, 10^{19} \mathrm{~cm}^{-3}$, and $10^{13} \mathrm{~cm}^{-3}-\mathrm{s}$, respectively. This performance

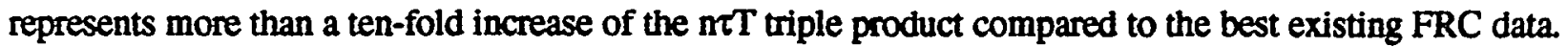
Los Alamos and the Air Force Research Laboratory in Albuquerque (Phillips site) form the experimental team who will develop the FRC plasma target and conduct liner experiments using primarily LANL experimental facilities for FRC target development and the AFRL Shiva-Star facility for energetic liner implosions.

In summary, MTF embodies an exciting new direction for fusion energy development at small scale and low cost. It enables the study of plasma confinement physics over a relatively unexplored region of density and magnetic field strength with a view to advancing CT physics in particular, and fusion energy research in general. The readiness for an MTF Proof-of-Principle program is predicated on the maturity of compact toroid physics and liner implosion technology, as well as the potential to achieve significant plasma performance with existing facilities at modest cost. Given the current circumstances that have led to a restructured fusion program, in which innovations with lower-cost development are encouraged, we believe the logic of investigating MTF is both propitious and compelling. 


\section{PART A: MOTIVATION AND GOALS}

\section{Motivation: A low-cost approach to fusion energy development.}

Los Alamos is leading a multi-institutional team that proposes to develop a completely new direction for fusion energy called Magnetized Target Fusion (MTF). We specifically propose to form and preheat a compact-toroid plasma using well-established techniques developed over the past 20 years, and then compress it with imploding liner technology developed by DOE and DOD defense programs. We will explain why our particular approach offers the most direct path for proving the principle of MTF, but we acknowledge that there are many additional issues involved with MTF as discussed in a Community Based R\&D Roadmap located at URL: http:/fusionenergy.lanl.gov/R\&DRoadmap.pdf

The technical reasoning that predicates MTF as a faster and cheaper approach to fusion energy development was recently published (see Appendix A). Basically, the reasons are threefold. First, fusion reactivity scales as density squared, which implies that unlike conventional magnetic fusion energy (MFE), energy gain can be achieved at high density in a short duration pulse. Second, all characteristic plasma scale-lengths (e.g. mean free path, gyro radius at fixed beta, $c / \omega_{p}, \lambda_{\text {debye }}, \ldots$ ) decrease with density. Consequently, the required plasma energy investment to achieve net fusion energy gain $(Q>1)$ decreases as a function of increasing plasma density. This statement is true for many different diffusive transport mechanisms (impurity radiation puts a limit on the acceptable impurity fraction independent of density). Third, the required power to achieve fusion-relevant conditions, roughly $1-10 \mathrm{TW}$, is much reduced compared with inertial confinement fusion (ICF), and readily available with existing pulsed-power facilities. Minimum power heating requirements can be seen as a function of density by noting Minimum Power $\sim$ (Minimum Energy) $/ \tau_{E}$, where $\tau_{E}$ is the energy confinement time consistent with fixed $n \tau_{E}$.

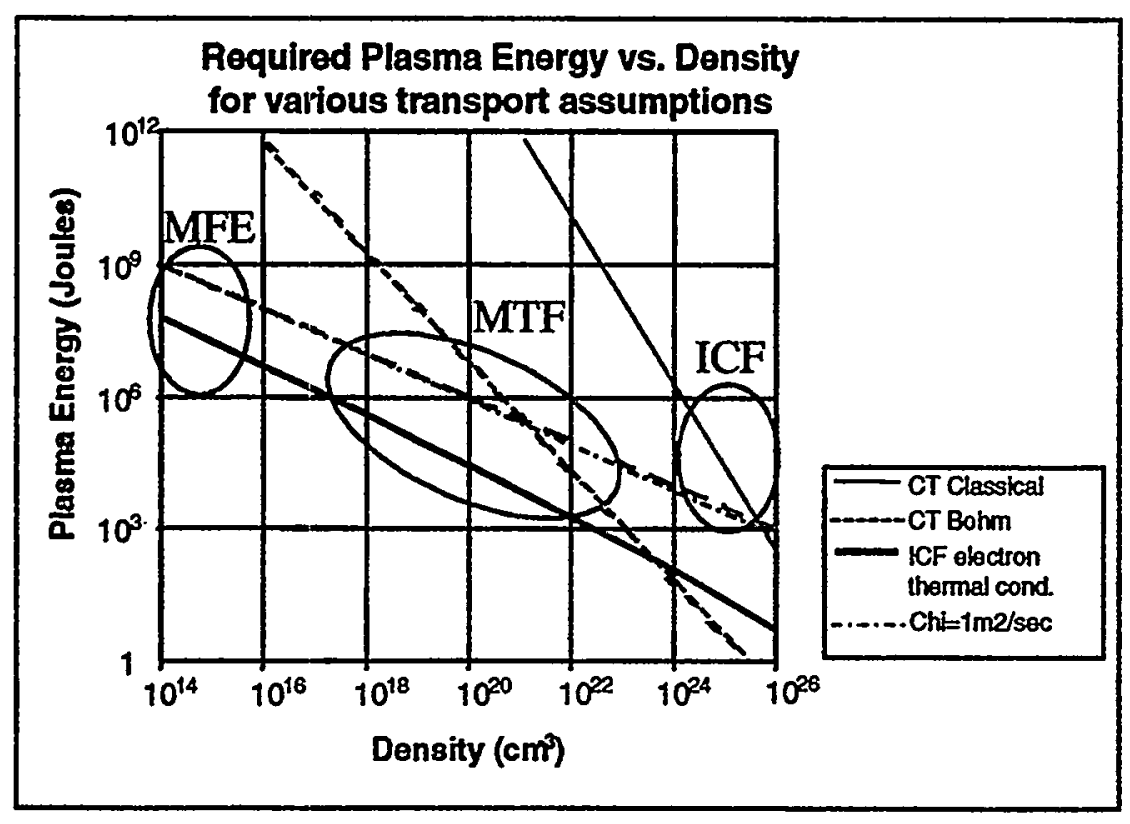

Fig. 1. Required thermal energy in a plasma to achieve Lawson $\left(n \tau=3 \times 10^{14} \mathrm{sec} / \mathrm{cm}^{3}\right)$ as a function of density at $\mathrm{T}=10 \mathrm{keV}$, assuming a sphere of unmagnetized plasma for ICF, and an FRC geometry with plasma $\beta=1$ for magnetized plasma. 
Figure 1 plots the minimum required thermal energy in a plasma to achieve Lawson $\left(\mathrm{n} \tau=310^{14} \mathrm{sec} / \mathrm{cm}^{3}\right.$ ) as a function of density for several thermal transport models. Here, poloidal $\beta$ of 1 and plasma temperature of $10 \mathrm{keV}$ is assumed, where $\beta=$ plasma pressure/magnetic pressure. For the case of diffusivity $\chi \sim 1 \mathrm{~m}^{2} / \mathrm{sec}$, typical of many tokamak experiments, the required thermal energy for breakeven must exceed roughly 1 GJ (approximately ITER). More interesting is the consequence of Bohm diffusivity. As shown in Fig. 1, there is a regime of density at around $10^{20} \mathrm{~cm}^{-3}$ where Bohm is good enough to achieve break-even with a very achievable energy investment. This is in strong contrast to conventional MFE densities, where Bohm is unacceptable in terms of the required energy. Unmagnetized plasma has the strongest scaling with density. Accordingly, ICF seeks to work at the maximum possible density where the required energy investment is small. The parametric scalings of required energy and power to achieve fusion breakeven all lead to the same conclusion: systems intermediate between present MFE and ICF allow for a lost-cost, fast-track development of fusion energy at modest scale. This addresses the critical problem of development cost confronting the worldwide fusion energy program. Given the current circumstances that have led to a restructured fusion program, in which innovations with lower-cost development are encouraged, the logic of investigating this new direction for fusion energy is compelling. Based on this thinking we have adopted the following mission for our MTF Proposal:

\section{Mission of the MTF Proof-of-Principle Program}

To explore the intermediate-density pathway to fusion conditions by liner compression of a compact toroid target, and to demonstrate the effectiveness of magnetic insulation for reducing driver requirements, thereby opening potential avenues to low-cost energy-producing plasmas and practical fusion power.

The target plasma will be compressed using a liner driver that symmetrically implodes the target to modest radial convergence $(-10)$. Sophisticated numerical models to describe liner implosions have been developed and benchmarked by experiments. To understand the issues in an elementary fashion, one starts by considering conservation of liner momentum and energy in a three dimensional compression [Siemon97]. Here, momentum is given by:

$$
\left.\delta_{o} \rho_{o} v_{L}=\text { [Peak pressure } x \tau_{D}\right]\left(R_{f} / R_{o}\right)^{2}
$$

where $\delta_{o}$ is the initial liner thickness, $\rho_{o}$ is the initial liner density, $v_{L}$ is the imploding liner velocity, $R_{f}$ is the final liner radius, $R_{0}$ is the initial liner radius, $\tau_{D}$ is the dwell time required for liner momentum to be stopped by peak pressure, and peak pressure is $2 n_{\mathrm{f}} \mathrm{k} \mathrm{T}_{\mathrm{f}}$ (plasma pressure and magnetic pressure are equal for the FRC case being considered). It is interesting to note that conservation of momentum provides a physical interpretation of the triple product (pressure times dwell time) in terms of initial liner momentum per unit area $\left(\delta_{\mathrm{o}} \rho_{\mathrm{o}} \mathrm{v}_{\mathrm{L}}\right)$. Obviously, this equation plays a fundamental role in designing a liner for the purposes of thermonuclear fusion. Liner energy is given by:

$$
2 \mathrm{n}_{\mathrm{f}} \mathrm{kT} \mathrm{T}_{\mathrm{f}}=(\varepsilon / 3 \mathrm{G})\left(\mathrm{R}_{\mathrm{o}} / \mathrm{R}_{\mathrm{f}}\right)^{2}\left(\delta_{\mathrm{d}} / \mathrm{R}_{\mathrm{f}}\right) \rho_{\mathrm{o}} \mathrm{v}_{\mathrm{L}}{ }^{2}
$$

where $\varepsilon$ is the fraction of liner kinetic energy converted to thermal energy at peak compression, and $G$ is the geometric ratio of plasma volume to the product of surface times radius for an arbitrary shaped plasma. Peak pressure, or density at $10 \mathrm{keV}$ temperature, is proportional to initial liner kinetic energy 
density $\left(\rho_{0} v_{L}{ }^{2}\right)$. The coefficients are geometric in nature and not the essential quantities. The essential requirement to create high-density plasma at $10 \mathrm{keV}$ temperature is a liner velocity of order a $\mathrm{cm} / \mu \mathrm{s}$.

Detailed calculations take into account other constraints such as liner compressibility and heating by the currents used to accelerate the liner. On the basis of calculations ranging from spreadsheet descriptions using global conservation laws described above, to time dependent coupled circuit and plasma models, we have established the quantitative goals for our PoP experiment described below.

\section{Proof-of-Principle Goals:}

The PoP research discussed in this proposal is focused on establishing a scientific basis from which the potential of MTF, as an attractive fusion power source, can be evaluated. For our approach of liner implosion of a CT target, the critical scientific plasma physics issues to address are:

- formation at required density $\left(-10^{17} \mathrm{~cm}^{-3}\right)$ and temperature $(-300 \mathrm{eV})$,

- stability during formation and compression,

- energy confinement adequate for fast liner compressional heating,

- plasma impurity content and the general consequences of high-energy-density plasma-wall interactions

The fourth critical technical issue of interactions between the plasma and an imploding liner wall depends in detail upon the target plasma magnetic configuration. We have chosen the FRC, in part, because it allows a continuum of possibilities. An FRC has a well-defined separatrix and a sheath of magnetic flux that separates it from the surrounding metal boundary. The flux external to the separatrix acts as a divertor for wall-related impurities. The radial position of the separatrix can be adjusted by the amount of trapped flux external to the separatrix. This provides a "knob" to evaluate questions of plasma-wall interactions and the various equilibria and transport phenomena that happen when plasma pressure is directly supported by a mechanical wall. The breadth of this physics from a theoretical perspective is given in Appendix C. The scientific issues here have elements in common with those of tokamak divertors, albeit with a much higher central density.

Broadly stated, the research effort consists of four phases:

1. Formation of an FRC using well-established theta-pinch technology at parameters suitable for compression. Based on the existing FRC knowledge base, as discussed in Parts B and C, the formation goals are $\mathrm{n} \sim 10^{17} \mathrm{~cm}^{-3}, \mathrm{~T} \sim 300 \mathrm{eV}, \mathrm{x}_{8}$ (normalized separatrix radius) $\sim 0.5-0.8$, $\mathrm{I}_{\mathrm{s}}$ (separatrix length) $\sim 30 \mathrm{~cm}$, and $\tau_{\mathrm{E}}>10 \mu \mathrm{s}$.

2. FRC translation and trapping into a liner suitable for compression. We have designed the FRC to remain within empirically-established operational limits with the objectives of improving the understanding of FRC stability and achieving good confinement during compression. This requires translation of the formed FRC into an elongated flux-conserving liner with dimensions $\mathrm{r} \sim 5 \mathrm{~cm}$ and $\mathrm{L}>30 \mathrm{~cm}$.

3. Demonstration that an elongated liner can be imploded in vacuum with a radial convergence of 10 and velocity exceeding $0.3 \mathrm{~cm} / \mu \mathrm{s}$. Defense programs research has demonstrated stable implosions of the required convergence and velocity with length to diameter ratios of roughly 1 (Part 
B-2). Here, we apply that technology to demonstrate implosions with length to diameter ratios of between 3 and 4 .

4. Integrated compression experiments where the formed and translated FRC is compressed by an imploding liner. The goal of this research phase is to prove the MTF principle for a specific plasma target system. That is, substantial heating and increased $\mathrm{nt}$ will result from liner implosion of a wellformed FRC plasma according to present understanding of plasma physics. We expect to heat the FRC to temperatures of $-5 \mathrm{keV}$, and in the process achieve $\mathrm{n \tau} \sim 10^{13} \mathrm{sec} / \mathrm{cm}^{3}$. This performance represents more than a ten-fold increase of the $\mathrm{ntT}$ triple proctuct compared to previous FRC experiments done without liner implosion. An important component of this objective is to advance fusion science by theoretical understanding and computer modeling of the experimental results.

The principal goals, scientific issues, and requirements of the proposed research plan are summarized in Table 1.

Table 1. Summary of research goals, issues, and requirements for MTF PoP Research

\begin{tabular}{|c|c|c|c|c|}
\hline Research Goals & Scientific/Technical Issues & $\begin{array}{l}\text { Principal } \\
\text { Facility }\end{array}$ & Principal Diagnostics & $\begin{array}{l}\text { Modeling } \\
\text { Codes }\end{array}$ \\
\hline $\begin{array}{l}\text { FRC formation Suitable for } \\
\text { Compression } \\
\mathrm{T} \sim 300 \mathrm{eV} \\
\mathrm{n} \sim 10^{17} \mathrm{~cm}^{-3} \\
\tau_{\mathrm{E}}>10 \mu \mathrm{S}\end{array}$ & $\begin{array}{l}\text { FRC formation at high density } \\
\text { Stability for } S^{*} / E<3.5 \\
\text { Confinement } \tau_{E}-0.5 R^{2} / \rho_{1} \\
\text { Impurity Content } Z_{\text {eff }}<2\end{array}$ & $\begin{array}{l}\text { Colt } \\
\text { (LANL) }\end{array}$ & $\begin{array}{l}\text { Excluded flux } \\
\text { B probe array } \\
\text { Interferometry } \\
\text { Thomson scattering } \\
\text { Bolometry } \\
\text { Optical spectroscopy }\end{array}$ & $\begin{array}{l}\text { Analytic(0D) } \\
\text { MOQUI }\end{array}$ \\
\hline $\begin{array}{l}\text { FRC Translation and } \\
\text { trapping into a liner with } \\
r_{\text {wall }}=5 \mathrm{~cm}, \\
r_{8}-3 \mathrm{~cm} \\
l_{8}=30 \mathrm{~cm}\end{array}$ & $\begin{array}{l}\text { Maintaining stability } \\
\text { Impurity content }\end{array}$ & $\begin{array}{l}\text { Colt } \\
\text { (LANL) }\end{array}$ & $\begin{array}{l}\text { B probe array } \\
\text { Bolometry } \\
\text { Interferometry } \\
\text { Spectroscopy }\end{array}$ & $\begin{array}{l}\text { Analytic(OD) } \\
\text { MOQUl }\end{array}$ \\
\hline $\begin{array}{l}\text { Vacuum liner compression } \\
\text { from } r_{\text {intes }}=5 \mathrm{~cm} \text { to } \\
r_{\text {fimut }}=0.5 \mathrm{~cm} \\
\text { with } V>0.3 \mathrm{~cm} / \mu \mathrm{s}\end{array}$ & $\begin{array}{l}\text { Rayleigh-Taylor and kink } \\
\text { stability for } L / D \sim 3 \\
\text { Convergence } R / R_{f} \sim 10\end{array}$ & $\begin{array}{l}\text { Shiva-Star } \\
\text { (AFRL) }\end{array}$ & $\begin{array}{l}\text { 3-axis radiography } \\
\text { end-on framing photos } \\
\text { magnetic probe } \\
\text { pin arrays }\end{array}$ & $\begin{array}{l}\text { CONFUSE(OD) } \\
\text { RAVEN(1D) } \\
\text { MACH-2(2D) } \\
\text { RMHD(2D) } \\
\text { LASNEX(2D) } \\
\end{array}$ \\
\hline $\begin{array}{l}\text { Integrated Compression of } \\
\text { FRC in liner to } \mathrm{T} \sim 5 \mathrm{keV}, \\
\mathrm{n} \sim 10^{19} \mathrm{~cm}^{-3} \tau_{\mathrm{B}} \sim 1 \mu \mathrm{s}\end{array}$ & $\begin{array}{l}\text { Stability } \\
\text { Transport } \\
\text { Impurity content (liner mix) }\end{array}$ & $\begin{array}{l}\text { Shiva-Star } \\
\text { (AFRL) }\end{array}$ & $\begin{array}{l}3 \text { axis radiography } \\
\text { End-on interferometry } \\
\text { Spectroscopy } \\
\text { Bolometry } \\
\text { Neutron emmsion }\end{array}$ & $\begin{array}{l}\text { MSG (OD) } \\
\text { MAGIT (OD) } \\
\text { MHRDR (1D) } \\
\text { RAVEN(1D) } \\
\text { RMHD(2D) } \\
\text { TRAC-II } \\
\text { ASCl codes }\end{array}$ \\
\hline
\end{tabular}




\section{The PoP team and budgets.}

For the first three years, the multi-institutional tasking and the estimated total budget is summarized in Table 2. While reactor considerations are not central to a PoP effort, we include about $8 \%$ of this effort to examine the issues of practical energy application.

Table 2. Budgets and tasks for each institutional team member.

\begin{tabular}{llc}
\hline \multicolumn{1}{c}{ Institution } & \multicolumn{1}{c}{ Task } & $\begin{array}{c}\text { Annual budget for 3-year PoP } \\
\text { (\$millions) }\end{array}$ \\
\hline LANL & $\begin{array}{l}\text { CT plasma formation development } \\
\text { CT Integrated compression } \\
\text { experiments }\end{array}$ & 3.0 \\
AFRL & CT Liner-prototype development \\
(Previously Phillips & Integrated compression experiments \\
Laboratory) & Impurity measurements/assessment \\
LANL & CT liner design & 1.0 \\
& $\begin{array}{l}\text { Plasma and liner integrated } \\
\text { modeling }\end{array}$ & \\
& Systems studies & 1.1 \\
LLNL & Plasma-liner interaction theory and \\
& modeling & \\
System studies & 0.4 \\
General Atomics & Theory of wall-plasma interactions \\
U. Washington & FRC MHD modeling & 0.1 \\
Westinghouse & Energy systems analysis & 0.1 \\
University supporting & Related exploratory concept \\
research & development & 0.3 \\
\hline & Total & 0.6 \\
\hline
\end{tabular}




\section{PART B: KEY SCIENTIFIC ISSUES}

In this section we summarize, for our specific approach, the key scientific issues for the development of MTF as a fusion coneept. For each issue, the status and open issues are discussed with a view to defining a PoP research program. In our proposed research plan, we have chosen a compact toroid for the target plasma. Compact toroids (CTs) offer the promise of robust, closed flux surfaces that maintain their topology during compression. After considering both the field-reversed configuration and spheromak types of CTs, as well as other candidate target plasmas (see Appendix B), we have selected the fieldreversed configuration (FRC) as our principal CT target candidate for the reasons given below. The required quantitative parameters for FRC targets can be approximated assuming adiabatic compression during liner implosion, but we base the estimates here on a Zero-D liner-implosion model described at the beginning of Part $\mathrm{C}$.

\section{Plasma Target: The Field Reversed Configuration}

a. Background. The FRC is an elongated compact toroid (Fig. 2) that is formed without toroidal field [Tuszewski88]. The FRC consists of a closed-field-line torus inside a separatrix and an open-field-line sheath outside the separatrix. Equilibrium in a FRC is a balance of magnetic field pressure and plasma pressure in the radial direction, and field-line tension and plasma pressure in the axial direction. Thus, in a straight cylinder, it follows as first derived by Barnes [Barnes79], that average pressure inside the separatrix is $\langle\beta\rangle=1-x_{t}{ }^{2} / 2$, where $x_{s}=r_{d} / r_{c}$, and $r_{c}$ is the effective coil (or conducting wall) radius.

FRC research is actively pursued worldwide [see ICC white paper by Hoffman et. al.]. Fundamental properties of FRCs are closely related to the field-reversed sheath observed in the solar down-wind tail of the magnetosphere [Horton94]. Significant progress has been made in FRC experiments and theory, resulting in stable plasmas with good confinement properties.

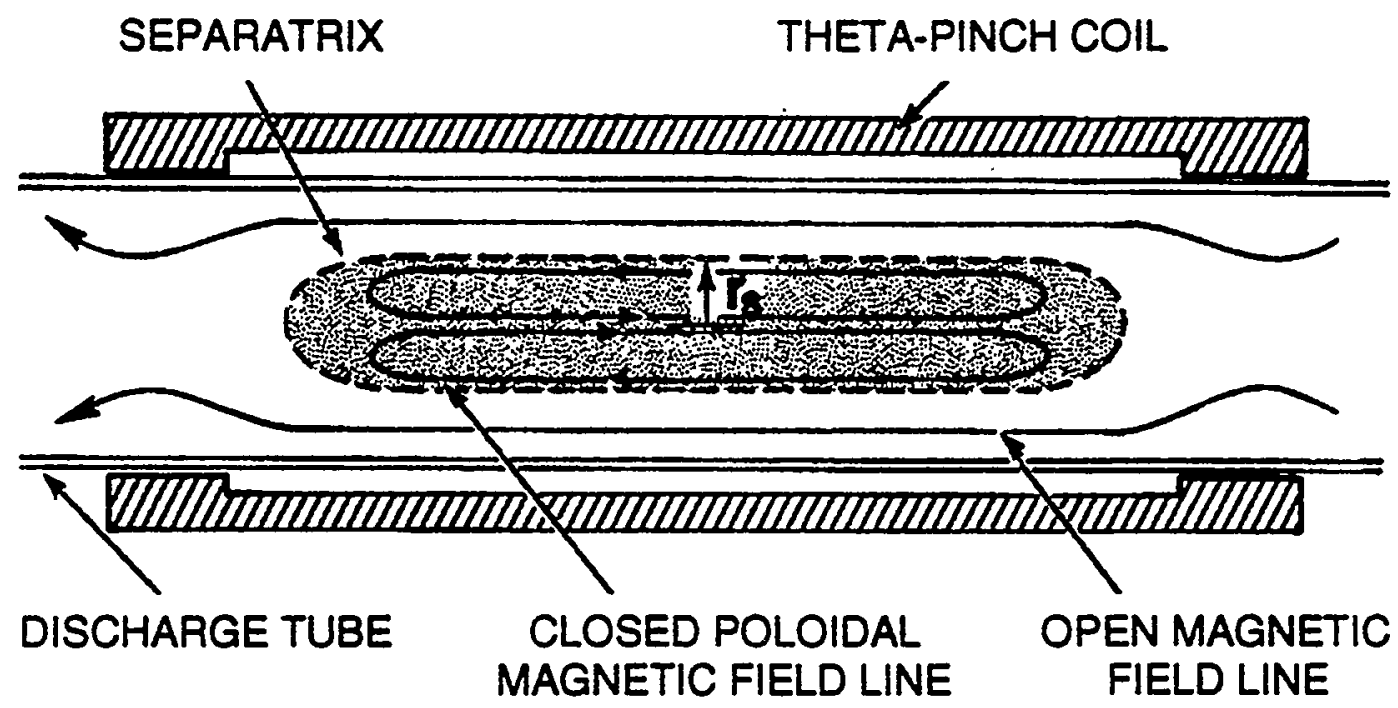

Fig. 2. FRC geometry showing separatrix radius and flux-conserving boundary.

The FRC is chosen for our proposed research because it offers many potential advantages as an MTF target plasma. The FRC is a compact torus, and therefore it has a closed-field-line topology without 
internal material objects. The compact torus configurations are ideally suited to liner compression. The FRC can be readily translated along its symmetry axis as has been demonstrated in many experiments. This permits the separation of plasma formation and liner-implosion regions. Formation of an FRC using high-voltage theta-pinch technology is well established, and appears likely to extrapolate to the required target requirements. The FRC is formed inductively and is largely free of impurity line radiation. Furthermore, the FRC separatrix is a natural divertor isolated from wall boundaries. The FRC has very high $(\sim 10)$ internal plasma beta, which allows efficient heating of plasma as opposed to magnetic fieid, and also serves to reduce substantially synchrotron radiation losses. The FRC undergoes an axial contraction during radial compression because of increased field line tension during compression. Thus, a purely cylindrical liner implosion results in 2.4-D compressional heating. A full 3-D compression could be achieved with shaped liners, if desired. By using the purely cylindrical liner, diagnostic access is improved during compression, which is an important feature for experimental progress, even if the energy requirements are somewhat increased. The FRC plasma length can be made smaller than the liner length, which may be important to avoid impurity influx from the liner ends. All together, the physics base developed by over twenty years of FRC research gives confidence that FRC targets have a reasonable chance of being compressed to fusion-relevant conditions.

b. Status of FRC formation. FRCs have been formed successfully during the last 40 years by the fieldreversed theta-pinch method. This technique has been improved in the last decade with more uniform preionization, non-tearing reconnection from end cusps, and axial dynamics control [Tuszewski88]. Successful FRC theta-pinch formation models have also been developed [Siemon80, Steinhauer83, and Tuszewski88b]. Other valuable FRC formation techniques are being developed, including coaxial slow sources, rotating magnetic fields, and formation by counter-helicity merging. However, the proven thetapinch FRC formation technique is a logical choice for our proposed research because formation and translation into the liner region can be accomplished in a few $\mu \mathrm{s}$; a time short compared to the expected FRC lifetime.

Most FRCs studied over the last 20 years were formed with pulsed-external magnetic fields, where $\mathrm{B}_{\mathrm{e}}<1 \mathrm{~T}$ and gas fill pressures $\mathrm{p}_{0} \sim 1-10 \mathrm{mTorr}$. These conditions yielded FRC plasma temperatures $\mathrm{T} \sim 0.1-1 \mathrm{keV}$ and plasma densities $\mathrm{n} \sim(1-5) \times 10^{15} \mathrm{~cm}^{-3}$. For MTF, FRC targets will require $\mathrm{B}_{\mathrm{c}} \sim 3-5 \mathrm{~T}$, $p_{0} \sim 200$ mTorr, yielding $\mathrm{T} \sim 300 \mathrm{eV}$ and $\mathrm{n} \sim 10^{17} \mathrm{~cm}^{-3}$. We note that FRCs with MTF relevant parameters were formed successfully in many early (1960's) and intermediate (1970's) theta-pinch experiments [Tuszewski88]. Although they were less well diagnosed than in more recent experiments, they give us good reason to believe that MTF target FRCs can be generated with the required parameters.

c. Open Issues of FRC formation. Compared with most previous FRC experiments, the relative amount of initial magnetic-piston heating (referred to as "implosion" heating in the literature) compared to resistive dissipation will be somewhat smaller in the FRC formation to be used here. The required initial temperature $(-300 \mathrm{eV})$ and trapped flux correspond to a bias field $\mathrm{B}_{0}=0.5-1.0 \mathrm{~T}$. An important issue is to check whether formation works as expected according to empirical models and two-dimensional numerical MOQUI calculations (detailed later) in the higher-density regime. Dominant resistive heating has been successfully demonstrated at low density in the TRX-2 device [Hoffman86].

A second issue is whether the fairly collisional regime of the initial FRC (ion mean free path $\lambda_{i} \sim 1 \mathrm{~cm}$ compared to separatrix radius $\mathrm{r}_{8} \sim 3 \mathrm{~cm}$ ) has the same favorable properties during formation as observed at 
lower density. A third issue is how rapidly the FRC can be formed, translated, and trapped in the liner volume. Given the fairly short lifetime expected, rapid translation is desirable. A $4^{\circ}$ conical theta pinch appears adequate [Rej86] to accelerate the FRC out of the formation region in about $2 \mu$ s with axial speed comparable to $\mathrm{V}_{\mathrm{A}} \sim 10 \mathrm{~cm} / \mu \mathrm{s}$. Two-dimensional MHD MOQUI simulations suggest that liner compression could be underway about $8 \mu \mathrm{s}$ after FRC formation. This time scale is less than the anticipated FRC lifetime of 20-30 $\mu$ s.

d. Status of FRC stability. Typical FRCs shows remarkable robustness with relatively good confinement properties over an established domain of dimensionless plasma parameters. The domain of good FRC stability and confinement is shown in Fig. 3 in terms of the size parameter $S^{*}$ and elongation parameter, E. Here, $S^{*}=r_{s} /\left(c / \omega_{\mathrm{pi}}\right)$, where $r_{\mathrm{s}}$ is the separatrix radius and $c / \omega_{\mathrm{pi}}$ is the ion electromagnetic skin depth, and $E=1_{g}\left(2 r_{8}\right)$, where $=1_{8}$ is the separatrix length.

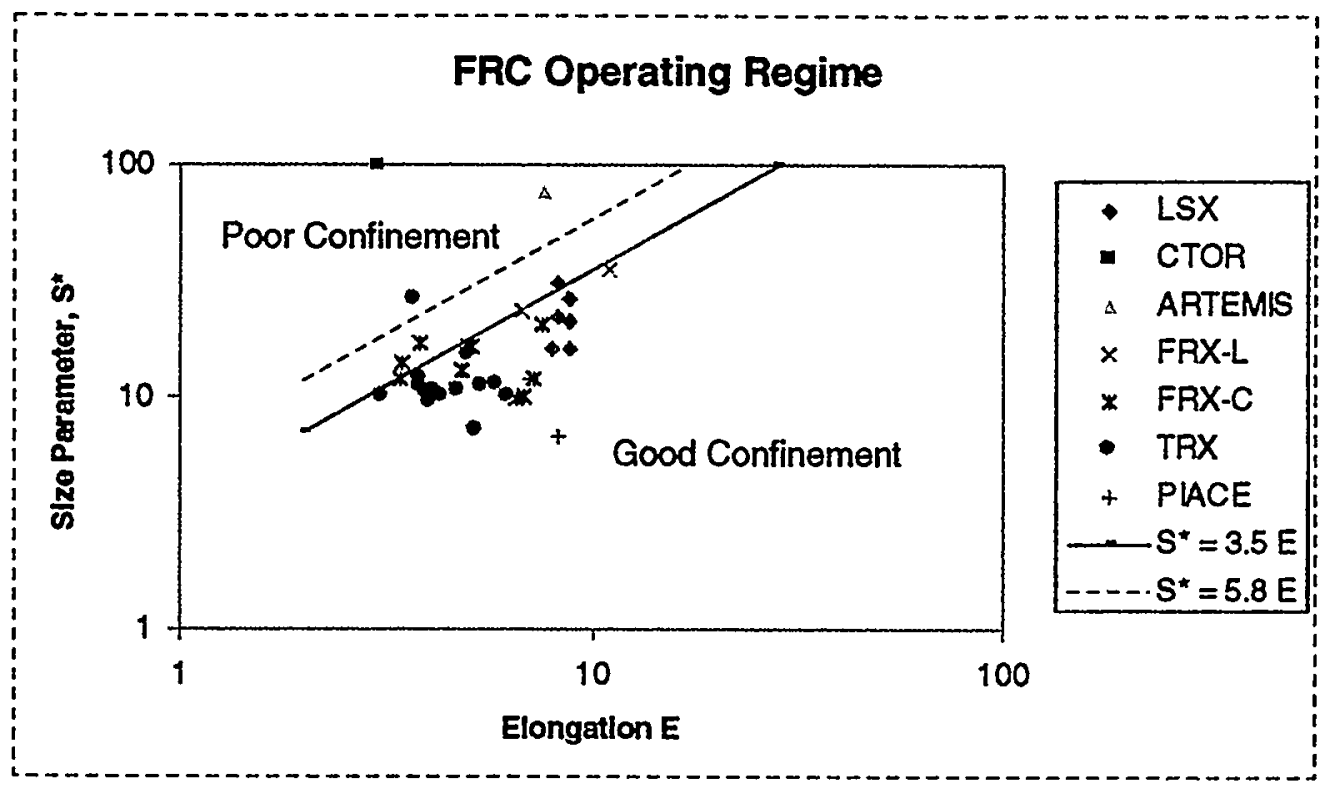

Fig. 3. FRC regime of good confinement.

These parameters arise naturally in FLR stability calculations. $S^{*}$ is approximately related to the small-S FRC stability parameter (often mentioned in earlier literature) by $S^{*} \sim 10 \mathrm{~s}$. The advantage of $S^{*}$ is that it can be easily defined experimentally using just excluded flux and side-on interferometry diagnostics. Most FRC experiments are observed to lie in the region $S^{*} / E<3.5$, that corresponds to the marginal finite-Larmor-radius FRC tilt stability limit with a gyroviscous ion fluid formalism [Ishida92]. This limit (depending on internal B profile) is also in agreement with significant tilt growth rate reduction seen in kinetic calculations [Barnes86].

There is no consensus, at present, whether the experimental data base shown in Fig. 3 reflects an FRC stability limit, whether it arises from global modes such as the internal tilt and/or from more localized modes such as interchanges, or whether it just coincides with technical limitations in FRC formation. A smooth transition from good to bad FRC confinement that correlates with increased Mirnov magnetic activity, has been observed when $S * / E>3-5$ in the FRX-C/LSM experiment [Tuszewski91a]. Signatures very similar to those in 3-D MHD tilt simulations were observed at low gas fill pressures, but higherorder mode activity dominated at higher gas pressures. The best results from the LSX device [Slough93, 
Hoffman93a], indicated in Fig.3, are also consistent with the empirical limit $S^{*} / E<3.5$. However, those FRCs showed high-order Mimov disturbances that did not clearly correlate with FRC confinement properties.

The experimental domain ( $(* / E<3.5$ in Fig. 3), where robust FRC's with good confinement properties are observed, remains to be definitively correlated with basic FRC stability theory. In addition, theoretical studies are underway [Ishida92, Steinhauer97] to clarify whether the $S^{*} / E$ stability limit can be pushed up to values substantially greater than 3.5 , either naturally from additional effects such as Hall and parallel kinetics, or from separatrix shaping and internal current hollow profiles. Valuable experimental research has been recently proposed to clarify the FRC stability domain in a wide $S^{*}$ and $E$ domain [Yamada98].

We adopt $S^{*} / E<3.5$ as a conservative empirical constraint on FRC formation, translation, and compression for the present proposal. Analytic adiabatic FRC compression laws [Spencer83] suggest that $S^{*} / E$ actually decreases during wall compression as roughly $r_{c}{ }^{0.4}$. On the other hand, $S^{*} / E$ is predicted by the same laws to increase significantly $\left(\sim x_{b}^{-2}\right)$ during flux compression. The latter result may explain the poor flux confinement observed in past flux compression experiments [Rej92]. For liner compression we will operate (FRX-L points in Fig. 3) safely below the conservative boundary.

e. Open issues of FRC stability. While the exact nature of the apparent $S^{*} / E$ limit shown in Fig. 3 remains uncertain, preliminary calculations, detailed later in this proposal, suggest that the fusion yield is very sensitive to the exact value of $S^{*} / E$ achieved while retaining good FRC confinement. An important physics issue for MTF, as well as for the international FRC community, is to determine how high $S^{*} / E$ can actually be. Fortunately, interesting liner compression experiments with significant fusion yield are possible with just $S^{*} / E<3.5$. Much better results may be obtained with somewhat higher $S^{*} / E$ values. Thus, determining the $S^{*} / E$ stability limit for MTF-grade plasma is an important experimental issue.

Another open issue is to what degree liner compression may be limited by the $n=2$ rotational instability that terminates many FRC experiments. This instability can be controlled with multipole fields, but this technique is probably impractical in a liner experiment. The $n=2$ rotational instability proved to be a limiting factor during some flux compression experiments [Rej92]. However, it proved rather harmless during translation experiments [Rej86], especially for cases with values of $x_{i}=r / r_{c}-0.6-0.7$. Wall compression proceeds with fairly constant $x_{t}$ values, unlike flux compression, so we plan to adjust the initial liner magnetic field to control $x_{8}$. Thus, it should be possible to avoid premature destruction of the compressed FRC from rotational instabilities.

f. Status of FRC transport. In the domain $\mathrm{S}^{*} / \mathrm{E}<3.5$ where robust FRC behavior is observed (Fig. 3), the FRC energy confinement time is approximately $\tau_{E}(\mu s) \sim 0.5 R^{2} / p_{i}(\mathrm{~cm})$, where $p_{i}$ is calculated with the external $B$ and internal $T_{i}$. [Siemon86, Tuszewski88, Hoffman93b]. This empirical relation was first suggested in transport calculations with anomalous resistivity from the Lower Hybrid Drift microinstability [Hamasaki79]. However, this microinstability has not been clearly identified [Carlson87].

Most FRC experiments indicate that the energy confinement is largely of convective nature, that is $\tau_{E}$ is dominated by particle loss [Tuszewski88, Steinhauer92, and Slough95]. FRCs typically show comparable particle $\tau_{N}$ and internal flux $\tau_{\phi}$ confinement times $\tau_{N}-\tau_{\phi} \sim R^{2} / p_{i}$. Nonconvective thermal losses are smaller in most FRC experiments, and appear mostly in the electron channel, presumably from a combination of thermal conduction and impurity radiation. 
FRC radial pressure balance yields $p_{i} \sim n^{-12}$, so that $S^{*} \sim R^{1 / 2}, \tau_{E} \sim R^{2} n^{1 / 2}$, and $n \tau_{E} \sim R^{2} n^{3 n} \sim S^{* 3} / R$. Past FRC experiments have typically achieved $n \tau_{E}$ values of a few $10^{11} \mathrm{~s}^{-\mathrm{cm}^{-3}}$ with $S^{*} \sim 20-40$ and $R \sim 5-10$ $\mathrm{cm}$. The empirical scaling law $n \tau_{E}-S^{*} / R$ suggests that large values of $n \tau_{E}$ can be achieved in a liner, with small values of $R$. For example, $n \tau_{E} \sim 10^{14} \mathrm{~cm}^{-3} \mathrm{~S}$ and $S^{*} / E<3.5$ corresponds to $S^{*} \sim 40, R-0.2 \mathrm{~cm}$, and $l_{s} \sim 5 \mathrm{~cm}$ in an FRC liner compression with $\mathrm{n} \sim 4 \times 10^{19} \mathrm{~cm}^{-3}$. We argue that achieving $\mathrm{n} \tau \sim 10^{13} \mathrm{~s}_{-} \mathrm{cm}^{-3}$ would prove the principle that high density is advantageous, assuming improved understanding of this density scaling is achieved during the research program.

It is interesting to note that $n \tau_{E} \sim 10^{14} \mathrm{~cm}^{-3} \mathrm{~s}$ value in a "conventional" FRC reactor with $\mathrm{n} \sim 10^{15} \mathrm{~cm}^{-3}$ would require $S^{*} \sim 300$ and $R \sim 1 \mathrm{~m}$. Meeting the constraint $S^{*} / E<3.5$ would require $\mathrm{l}_{\mathrm{s}}>100 \mathrm{~m}$ for those FRC reactor cases. The design burn points of two FRC reactor studies, CTOR [Hagenson81] and ARTEMIS [Momota92] are indicated in Fig. 3 to illustrate the previous statement. These design points lie well outside of the $S^{*} / E<3.5$ domain because relatively small FRC lengths (10's of meters) were chosen in those low-density reactor studies to keep the overall reactor power under a gigawatt.

g. Open issues of FRC transport. While empirical FRC scaling is well documented, transport properties are not well understood from the perspective of fundamental physics. This was the case a decade ago [Tuszewski88] and remains the case today. We assume in the present proposal that the above empirical scaling laws apply during the entire FRC liner compression. Zero-dimensional (0-D) calculations, detailed later in this proposal, suggest that significant particle losses do not substantially affect the overall fusion performance because of a concomitant increase in axial heating.

A key question is whether the FRC confinement properties of past experiments with $T_{e}<500 \mathrm{eV}$ and $T_{i}<$ $2 \mathrm{keV}$ will apply near peak compression where $T_{e} \sim T_{i} \sim 5 \mathrm{keV}$ is anticipated. Will thermal losses remain mostly convective, or will non-convective losses dominate? Electron thermal conduction may prove to be a limiting factor. Impurity radiation is another key issue for MTF that will be discussed in the next Section. It is also possible that ion thermal conduction may become a dominant energy loss mechanism if ion temperature gradients comparable to $r_{8}$ arise from a cold boundary. Control of $x_{6}$ may prove essential to avoid large $T_{i}$ gradients. Alternately, increasing $x_{i}$ to values approaching unity may increase thermal conduction but suppress density-gradient-driven convective losses.

h. Status of FRC impurity content and radiation loss during formation. Most FRCs produced by the field-reversed theta-pinch technique show relatively clean plasmas. This is presumably because of the inductive plasma formation where wall contact is limited to a few 100 ns during field reversal. After that the FRC separatrix is well separated radially and axially from the walls. FRCs intrinsically tend towards cleanly formed magnetically confined plasma.

Radiation losses have been occasionally measured or inferred in FRC experiments to be 5 - 10\% of the total energy losses [Tuszewski88]. Somewhat higher radiative loss fractions have been measured in the slower formation LSX experiment [Slough92]. In all cases, $Z_{\text {eff }}$ values in the range $1-1.5$ have been inferred. Line radiation is consistent with either $<1 \%$ oxygen or $\sim 0.04 \%$ Si concentrations, the dominant wall species of the quartz liner used for formation.

The impurity content of the proposed FRCs are likely to be comparable or less than in previous experiments. Somewhat larger bias fields may cause larger quartz impurity release, but the gas fill 
pressures and the final FRC densities are two orders of magnitude higher than in recent FRC experiments. Hence, the impurity concentrations are likely to be smaller.

i. Open issues of FRC Impurities during formation. Time-dependent calculations are needed to assess the impact of line radiation on FRC formation at MTF-relevant parameters. Even small concentrations of high-Z impurities may yield significant radiation power losses. However, it is likely that most important impurity concerns will arise from the liner-plasma interactions during compression rather than from initial FRC target plasma formation.

\section{Liner Implosions: DP Developed Capabilities}

a. Background. The broad scientific utility of high-speed liners with large kinetic energy has led to significant investment in liner physics studies and liner technology advancement. High-performance liners have, to date, been imploded by 5-15 MA of current from capacitor banks and greater than $100 \mathrm{MA}$ of current from explosively powered flux compressors. Currently, operational capacitor bank facilities include Pegasus (4.5 MJ) at Los Alamos and Shiva-Star (9.2 MJ) at the Air Force Research Laboratory (AFRL) in Albuquerque. Under DOE sponsorship, Los Alamos is designing and constructing the $24 \mathrm{MJ}$ Atlas facility with anticipated operation in 2001. Explosive flux compression generators proctucing 10-20 MJ are operational at Los Alamos and 50-100 MJ systems are in development. Similarly, explosive pulse power systems with greater than 100 MJ have been demonstrated at the All Russian Scientific Research Institute of Experimental Physics (VNIIEF) in Sarov, Russia. These systems have successfully imploded near-solid-density metal liners to MTF-relevant velocities. Shiva-Star is expected to be sufficient for the initial PoP implosion experiments envisaged in this proposal as discussed in Part C. Atlas and the explosive pulse power systems are expected to be adequate for follow-on proof-of-performance class experiments in the future. Thus, wide ranges of power systems are and will be available to meet the needs of MTF experiments.

b. Status of implosion liner performance. The axial z-pinch provides an effective and convenient configuration for driving liners with pulsed electrical energy. Here, the driving current is applied in the axial direction, giving rise to azimuthal magnetic fields outside the liner. Practical time-scales (microseconds), coupled with normal metal resistivity, $(2-10 \mu \Omega-\mathrm{cm})$, insure that the current distribution is limited to the outer surface at early times. For example, the magnetic diffusion skin depth in aluminum $(2.7 \mu \Omega-\mathrm{cm})$ is about $0.3 \mathrm{~mm}$ in $1 \mu \mathrm{s}$ for a step increase in the vacuum field. The currents and fields diffuse into the millimeter thick conducting liner during the course of the implosion. Implosion velocities of $1 \mathrm{~cm} / \mu \mathrm{s}$ have been demonstrated on present facilities while maintaining most of the liner at or above solid density, and while maintaining a significant fraction of the liner material below ambient melt temperature [Reinovsky96]. This velocity performance is sufficient for our proposed MTF PoP integrated compression experiment:

The drive pressure in the z-pinch configuration is applied to the outer surface of the liner by the magnetic field in a manner similar to a high-pressure but mass-less fluid. The acceleration of the outer surface (interface) is directed from the low-density (mass-less) fluid into the higher-mass-density metal liner. This is the magnetic analogy of the classical Rayleigh-Taylor (RT) fluid interface instability with an Atwood number equal to one. While the development of flute instabilities $(m>0)$ in the cylindrical liner is inhibited by the azimuthal driving field, the development of sausage instabilities $(m=0)$ on the outer surface poses an ultimate limitation on achievable liner convergence. Nevertheless, because material 
strength of solid liners inhibits instability growth, a convergence sufficient for an MTF PoP experiment $(\sim 10)$ is achievable with present liner-driver technology.

For the hydrodynamic RT instability, it has been shown analytically that fluid strength can recuce the growth rate of sufficiently small (wavelength and initial amplitude) perturbations as long as driving pressure is less than or the same order as the material strength [Chandrasekhar61]. The case of the magnetohydrodynamic RT is more complicated because the large currents needed to achieve interesting accelerations heat, and ultimately melt, part of the liner material, recucing or perhaps eliminating both the yield strength and shear modulus in the heated part of the liner. Furthermore, as the current diffuses into the liner, the time dependent behavior of material strength has a spatial dependence as well. Numerous analytic models of the hydrodynamic RT have been presented [Miles66, Drucker80, and Lebedev93] and some detailed comparisons with experiments have also been performed [Lebedev93, Robinson89, and Barns74].

Since 1994, an extended family of experiments have been conducted in the Los Alamos High-EnergyDensity Physics (HEDP) program to explore the physics issues associated with accelerating liners. The high-precision liners developed in these experiments have also been applied to drive a variety of hydrodynamic and material-property experiments such as those identified above. Nearly 100 separate experiments have been performed on the Los Alamos Pegasus capacitor bank at driving currents up to 12 MA (0.5-2.5 MJ of stored energy) and velocities exceeding $10 \mathrm{~km} / \mathrm{s}$. In these experiments, a 3-gram aluminum liner (0.4-mm initial thickness) was accelerated to velocities from 0.4 to about $1 \mathrm{~cm} / \mu \mathrm{sec}(50$ $100 \mathrm{~kJ} / \mathrm{cm}$ ). A significant number (approximately 15) of the Pegasus experiments were focused on of the RT instability of an imploding liner. Experimental results, with pre-imposed simusoidal perturbations (1050 micron amplitude and $0.5-6 \mathrm{~mm}$ wavelength), were in notable agreement with both the analytic and computational predictions of perturbation growth in both the elastic-plastic and fully melted fluid regime.

A limited number of very large-scale liner physics experiments have been performed jointly with VNIIEF, using explosively powered flux compression generators at 50-100 MA (25-50 MJ). In the largest of these experiments, a $1-\mathrm{kg}$ aluminum liner was accelerated to $0.8 \mathrm{~cm} / \mu \mathrm{sec}(32 \mathrm{MJ}$ of kinetic energy at $3-5 \mathrm{MJ} / \mathrm{cm}$ ). The liner initial radius was $20 \mathrm{~cm}$ and measurements were made at a radial convergence of about $4: 1$. The final radius in this experiment $(5-6 \mathrm{~cm})$ was specifically chosen to demonstrate liner performance appropriate for a large-scale MTF compression experiment. The Russian literature contains numerous references to comparable experiments, essentially demonstrating the reproducibility of high-current liner-compression techniques. During about the same period, several experiments with shaped- and mass-profiled liners conducted at the AFRL (10-15 MA currents at about 5 MJ stored energy) demonstrated the feasibility of axial mass contouring to achieve quasi-spherical implosions.

The combination of experience with imploding liners at kinetic energies ranging from 50 to $5000 \mathrm{~kJ} / \mathrm{cm}$, coupled with detailed comparisons between analytic theory, computational simulation, and experimental data for liner stability, provide reasonable confidence that liner technology appropriate for compression of MTF target plasmas is presently available.

c. Open issues of liner performance. The imploding liners appropriate for MTF compression experiments are similar to those being developed for HEDP experiments at Los Alamos. The HEDP research will contimue to develop the technology needed for (large) high-velocity liners (approaching 50- 
$100 \mathrm{MJ}$ kinetic energy). However, FRC-specific liners with larger length to diameter ratios, will require some development beyond liners for HEDP applications. Liner studies with bias magnetic field to determine the achievable compressed value of magnetic field in the specific geometry to be used for FRC compression are also important. Wall heating by eddy currents is severe for the mega-gauss fields to be used. Liner melting will reduce mechanical strength and increase electrical resistivity. These effects will limit the maximum fields that can be achieved by compression. Liner melting may also enhance impurity concerns and affect liner stability. Computational models to quantify these concerns are available and will need to be used and checked by liner experiments before integrated liner-on-plasma experiments are started. Also, liner-electrode interactions and the injection of metal vapor at the ends of the liner into the volume where plasma is to be compressed will require careful study. The proposed MTF program will include design and prototyping of liners appropriate to an MTF compression experiment based on the analytic, computational, and experimental databases from HEDP. For a selected driver/target configuration, the demonstration of required liner properties (velocity, symmetry, shape, vacuum integrity, and uniformity) will be a pre-requisite to a plasma compression experiment.

\section{Integrated Compression}

This first serious attempt to design and conduct integrated compression experiments depends upon target plasma development activities combined with the experience base of high-energy line implosion technology. The integrated experiments will provide extremely interesting data on imploding MTF systems. Experimental data is needed to support the computational evaluation of MTF as an approach to achieving fusion energy. While significant advances in MTF physics are expected from these first integrated compression experiments, it is not realistic to expect eariy experiments to reach high fusion reactivity. Rather, our goal is to develop a scientific basis for MTF from which its potential can be evaluated. This entails assessing liner-plasma stability, plasma impurity content, and plasma transport under fusion-relevant implosion conditions.

a. Status of liner-plasma interactions. A small number of FRC liner compression experiments were done on the TOR-liner device [Alikhanov83, Es'kov81]. An FRC was translated into a 5-cm-radius liner with the following estimated parameters: $B_{e} \sim 0.5 \mathrm{~T}, \mathrm{n} \sim 4 \times 10^{15} \mathrm{~cm}^{-3}, \mathrm{~T}_{\mathrm{e}}-\mathrm{T}_{\mathrm{i}}-50 \mathrm{eV}, \mathrm{r}_{\mathrm{s}}-4 \mathrm{~cm}$, and $\mathrm{l}_{\mathrm{s}}-$ $20 \mathrm{~cm}$. These parameters correspond to $S * / E \sim 3$. A medium-speed $(\sim 1 \mathrm{~mm} / \mu \mathrm{s})$ quasi-spherical liner compression (compression ratio $\sim 5-10$ ) was achieved with a shaped liner. A global neutron yield of about $2 \times 10^{8}$ was reported. Zero-D modeling [Es'kov83] confirmed that this neutron yield is consistent with volume compression ratios of about 1000 and plasma temperatures of $1.5-3.5 \mathrm{keV}$, depending on assumed cross-field transport. Unfortunately, very few plasma measurements have been reported and these encouraging results were not pursued.

b. Open issues of liner-plasma interactions. Our plasma compression scheme entails injecting a formed FRC into an imploding liner. A significant challenge is impurity release from the liner into the plasma target. Armstrong [Armstrong87] believed that ejecta from the liner walls prior to the FRC arrival, was an issue. However, he evaluated the ejecta for the case with explosive drive when shock waves reach the inner liner surface. Shocks and associated ejecta are minimized with the electric drive proposed here.

Understanding of liner issues described above, such as metal jets produced by the liner motion along the end glide-plane electrodes, will be critically tested in liner-on-plasma experiments. Obviously the issue of liner melting will also be important, both on the outside from the axial currents that drive compression, 
and on the inside from induced currents associated with the rising external field between the FRC separatrix and inner liner radius.

\section{Theory and Computation: Status and Issues}

a. Status. The proof of principle evaluation of MTF in a timely fashion and at minimum cost will require a strong synergism between experimental, theoretical, and computational efforts. We envision a cycle in which large-scale numerical computations guide experimental design, followed by detailed comparison of the experimental data with the corresponding computational predictions. Based on these comparisons, further development of the theory and modification of the computational tools will follow as appropriate. Finally, to complete the cycle, analysis of the experimental results will be used to guide the experimental evolution. This paradigm involving large-scale computation has been well proven in ICF and other DOE defense programs research activities.

Because of the dynamic nature of MTF experiments, extending the experiment/compute/experiment paradigm to MTF is optimum when both the plasma formation step and the liner implosion step are computed from $t=0$. That is, computations should begin at the plasma formation stage. The computations should include physical effects not normally considered in modeling of steady-state magnetized fusion systems. Examples of such effects include: material properties (equation-of-state, strength, resistivity, radiation, thermal conductivity, etc.) for solids, liquids, and partially ionized plasmas; shocks and dynamic non-equilibrium fluid flows; and convective energy loss from the plasma to its cold surroundings.

Fortunately, many computer codes already developed for ICF and other DOE programs are directly applicable to MTF research. For example, the LASNEX code (the computational tool that provides the theoretical basis for the national ICF program and the NIF) has confirmed that the potential parameter space for operation of magnetized targets is much more extensive than that for unmagnetized targets [Kirkpatrick91]. The MHRDR code (LANL), TRAC-II code (LLNL), and the MACH-2 code (AFRL) have predicted many of the observations (e.g., inductive probes, interferometry, $x$-ray emission, visible emission, neutron production) of the Russian MAGO plasma formation scheme that, to the extent that the computations are valid, has many of the properties required for a pre-implosion plasma [Lindemuth95]. The MACH-2 and TRAC-II codes have successfully modeled the RACE compact torus experiment. The one-dimensional code RAVEN and two-dimensional radiation magnetohydrodynamic (RMHD) computer codes have been used at Los Alamos to understand and interpret liner experiments in the implosion velocity range required for MTF [Faeh197]. The MOQUI code has been used to design FRC experiments and to interpret the behavior of FRC formation and translation from $t=0$ (Milroy82). A number of zerodimensional circuit/iner codes (eg. MSG, CONFUSE at LANL) are available to identify parameters for liner-on-plasma systems.

The strong synergism between MTF research and the on-going imploding liner research funded by DOE Defense Programs means that MTF will benefit from the major advancements in computing expected through the ASCI initiative. Already, ASCI codes are looking to pulsed-power-driven experiments, involving magnetic fields and magnetized plasmas, to contribute to the database required for code validation. Fully 3-D computer codes, which can model the combined liner and plasma dynamics of an MTF system, are expected to become operational in the same time frame as this proposal. 
b. Issues. An obvious technical challenge for MTF is enhanced radiation due to impurities derived from plasma-liner interactions. Little data and essentially no theoretical work are available to estimate the amount of impurities that can be introduced into the plasma from the possible sources that have been identified. Initial theoretical work suggests that MTF plasma may actually expel impurities from the plasma core [see Appendix C and Vekshtein90]. The short confinement times of MTF mean that wallconfined plasmas, or plasmas in very close proximity to the wall (i.e., pusher or liner), may be acceptable and desirable. The pioneering work at Columbia University [Gross75, Feinberg76] offers hope that transport from a hot plasma to a confining wall by thermal conduction may be "classical." However, 2-D computations [Lindemuth78] show that under certain circumstances, transport to the wall may be enhanced by 2-D convective flows. Understanding hot plasma interacting with a cold wall as discussed in Appendix $\mathrm{C}$ is rich in physical phenomena and is a principal focus of our proposed theory-modeling effort.

Mating a plasma formation system with a magnetically-driven imploding liner is the fundamental requirement of MTF. Considering the limited experience in this area, and recognizing that not all of the physics issues can be satisfactorily addressed by theory and computation without experiments, our goal is to move toward liner-on-plasma experimentation as soon as plasma formation and liner implosion are separately and satisfactorily understood. Computational modeling of the integrated compression experiments will be an essential component of understanding the complex physics of plasma stability and wall interactions. Codes for this purpose will involve modifications of existing and ASCI-class codes from DOE's Defense Programs. 


\section{PART C: RESEARCH PROGRAM}

We propose below an MTF PoP program based on the FRC target plasma. The experimental effort consists of three phases: (1) the FRC is formed in a conical theta pinch, (2) the FRC is translated and trapped in a liner suitable for implosion, and (3) the FRC is compressed by the liner to high temperature and density. To quantify the plasma parameters desired, we use a Zero-D model for FRC performance.

\section{Zero-D modeling of FRC performance.}

This 0-D model for cylindrical FRC liner (or wall) compression is based on past FRC research [Rej84] and is very similar to that used in an earlier evaluation of FRC liner compression [Armstrong87]. The calculation starts with an FRC at rest inside a liner and ends when a radial compression of 10 is achieved. The main assumptions of the model are:

(1) 2-D elongated FRC equilibrium at all times,

(2) thin liner with constant radial velocity $v_{\ell}$,

(3) $S * / E<3.5$ at all times,

(4) FRC transport: $\tau_{E}-0.5 R^{2} / \rho_{i}, \tau_{N} \sim \tau_{\phi}-R^{2} / \rho_{i}$, and

(5) liner resistivity and heating are considered.

The last assumption affects diffusion of the magnetic field in the sheath separating the liner from the FRC into the liner. The FRC formation requirements are calculated a posteriori, assuming identical FRC parameters after formation and before compression. This implies translation at constant external pressure and equal dimensions for the formation and initial liner regions. Similar translation has been demonstrated [Rej86]. An example of FRC parameters obtained from the 0-D model is given in Table 3.

\section{Zero-D Modeling parameters}

Liner assumptions: initial dimensions: 1-mm-thick alumimum, 30-cm-length, 10-cm-diameter mass: $250 \mathrm{~g}$

velocity: $0.3 \mathrm{~cm} / \mu \mathrm{s}$

kinetic energy: $1 \mathrm{MJ}$

compression time: $15 \mu \mathrm{s}$

dwell time $\left(r_{c} / v_{L}\right): 2 \mu s$

Equivalent DT fusion yield: neutron yield: $3 \times 10^{16}$

fusion energy: $0.1 \mathrm{MJ}$

$\mathrm{Q}$ (fusion/iner energies): 0.1

Efficiency (plasma/liner energies): 0.08

Conical theta pinch coil: radius: $4-6 \mathrm{~cm}$; length: $30 \mathrm{~cm}$

COLT Facility

voltage: single feed, $32 \mathrm{kV}$; electric field: $1 \mathrm{kV} / \mathrm{cm}$

main field risetime: $2.5 \mu \mathrm{s}$

deuterium gas fill pressure: 300 m'Torr

lift-off bias field: $0.8 \mathrm{~T}$ 


\section{Table 3. Zero-D calculations of FRC parameters}

\begin{tabular}{|l|c|c|}
\hline Parameter & before compression & after compression \\
\hline coil radius (cm) & 5 & 0.5 \\
\hline separatrix radius (cm) & 2.3 & 0.2 \\
\hline coil length (cm) & 30 & 30 \\
\hline separatrix length (cm) & 30 & 4.2 \\
\hline B external (T) & 5.4 & 520 \\
\hline peak density $\left(10^{17} \mathrm{~cm}^{-3}\right)$ & 1.2 & 350 \\
\hline$T_{e}(\mathrm{keV})$ & 0.3 & 8.6 \\
\hline $\mathrm{T}_{\mathrm{i}}(\mathrm{keV})$ & 0.3 & 10.6 \\
\hline plasma energy $(\mathrm{kJ})$ & 7.4 & 80 \\
\hline$\tau_{\mathrm{E}}(\mu \mathrm{s})$ & 28 & 4 \\
\hline particle inventory $\left(10^{19}\right)$ & 5.0 & 1.7 \\
\hline internal flux $(\mathrm{mWb})$ & 10 & 6.4 \\
\hline $\mathrm{S}^{*}$ & 23 & 35 \\
\hline $\mathrm{E}$ & 6.7 & 11 \\
\hline $\mathrm{S}^{*} / \mathrm{E}$ & 3.5 & 3.3 \\
\hline
\end{tabular}

Discussion and scaling arguments. These O-D calculations have tentatively identified an interesting PoP design point. The location of this design point before and after compression is indicated in Fig. 3 as FRX-L. The FRC initial state is chosen to satisfy $S * / E=3.5$. During compression, $S * / E$ decreases slightly, so that the FRC remains in the empirical domain of good FRC confinement. The ratio $S^{*} / E$ decreases less than predicted by the adiabatic scaling laws because of the significant energy losses in the example of Table 3 ( $\tau_{\mathrm{E}}$ only about twice the compression time). Reduced losses can be obtained with higher liner velocities: this would reduce the values of $S^{*} / E$ during compression but would also somewhat reduce the $Q$ values (increased liner energy).

The impact of the parameters $S^{*}$ and $E$ can be estimated by considering the scaling of the fusion yield for fixed temperatures and a fixed radial compression ratio. We assume that losses are small by requiring the initial FRC confinement time to exceed the compression time by a constant factor of 3 . The FRC final parameters scale as the initial parameters, so that we drop subscripts 1 and 2 . Then, the fusion yield $E_{f}$ $n^{2} V \tau_{d \text { well }}$. With $\tau_{\text {dwell }} \sim r_{\delta} / V_{L}, V \sim r_{8}^{2} I_{3}, S^{*} \sim r_{3}^{2} n^{1 / 2}$, and $S^{*} / E=c$, one obtains $E_{f} \sim c^{4} E^{5} /\left(x_{f} V_{L}\right)$. From $\tau_{E} \sim$ $\tau_{\text {dwell }}$ and $\tau_{E} \sim S^{*} r_{s}$, one further obtains $x_{8} v_{L} \sim 1 / S^{*}$. Hence, the fusion yield scales as $E_{f} \sim c^{5} E^{6}$, where $E$ is the FRC elongation before compression.

This expression shows the importance of maximizing $c$ in the FRC formation experiments that would occur in the first part of the project. If FRCs with good confinement properties can be obtained with $\mathrm{c}=$ 5.8 (dashed line in Fig. 3) rather than the assumed $c=3.5$ (solid line in Fig. 3), an order of magnitude increase in fusion yield could be obtained. This indicates that an important aspect of the proposed research coincides with a crucial issue in FRC research. Further insight into this issue, whether it is stability, confinement, or formation, will be gained during the proposed experiments.

The above fusion yield scaling also shows the importance of elongation $\mathrm{E}$. If FRC physics constrains $\mathrm{c}$ to a given value, $E_{f}$ can still be significantly increased by increasing the FRC elongation. For fixed liner 
dimensions, this implies low values of $x_{3}$, as in the example of Table 3. For fixed FRC separatrix radius before compression, this implies longer liners. Ultimately, $E_{\mathrm{f}}$ would be limited only by the magnetic field pressures that can be achieved at peak compression (B length).

\section{Target Plasma}

a. Target Plasma Goal. The principal goal of the first two years of experimental research will be to develop the formation of an FRC target plasma in a liner that is suitable for implosion. Specifically:

- forming an FRC with the properties shown in Table 3, and

- translating the formed FRC into a liner suitable for compression.

b. Target Plasma Approach. The first FRC formation experiments will be performed with a straight theta-pinch coil in a symmetric configuration without axial motion. Independent cusp coils at each end will be used to insure fast and symmetric non-tearing field line reconnection [Tuszewski88]. A thetapinch coil radius of about $5-\mathrm{cm}$ and a coil length of $30 \mathrm{~cm}$ may be used for the first experiments. A dielectric tube (quartz or alumina) of $4.5 \mathrm{~cm}$ radius will be inserted inside the coil. The coil would be connected to the existing Colt bank via collector plates and 40 load cables, yielding main field risetimes of 2-3 $\mu$ s. The reversed bias fields will be generated from a slow ignitron-switched capacitor bank (FRXC compression modules) connected to the coil collector plate.

FRC formation will be explored with external fields $B_{c}$ up to $5 \mathrm{~T}$, reversed bias fields of up to $1 \mathrm{~T}$, and deuterium gas fill pressures of 100 - 400 mTorr. Several key FRC physics issues will be explored during these preliminary formation experiments. These issues include FRC heating and internal flux retention, axial dynamics following field line connection, dielectric tube heating and possible impurity release during field reversal, FRC global energy confinement, stable period before the $n=2$ rotational instability, and FRC global stability as function of $S * / E$.

Most FRC formation issues can be assessed with two basic diagnostics: an excluded flux array that yields $\beta$ and $r_{8}(z)$, and a side-on laser interferometer to estimate the plasma density. Then, both $S^{*} \propto r_{8} n^{1 / 2}$ and $E$ $=1 /\left(2 r_{3}\right)$ can be obtained as function of time. The FRC particle confinement time can also be assessed with those two diagnostics. In addition, the excluded flux array will provide information on internal flux annihilation, axial dynamics during formation, and energy confinement during the equilibrium phase. The two diagnostics would also yield an estimate of the plasma total temperature $\left(T_{6}+T_{i}\right)$ from radial pressure balance. The exchuded flux array could be easily built with a single flux loop and an array of $\mathrm{B}_{\mathbf{z}}$ probes. Laser interferometers at $0.44 \mu \mathrm{m}$ ( $\mathrm{HeCd}$ ) and $3.39 \mu \mathrm{m}$ (HeNe) can be constructed using available Colt and old FRX-C hardware.

During the second year of the experimental program, other diagnostics will be used, such as single-point Thomson scattering to measure electron temperature and CV Doppler broadening and neutron yield, to estimate the ion temperature. Optical emission and bolometry are expected to estimate the impurity species and the radiated power. The latter may prove a crucial issue for the proposed FRC targets formed with bias fields of about $1 \mathrm{~T}$. Such large bias fields are likely to cause large thermal pulses [Steinhauer83] on the dielectric inner surface during reversal. Initial estimates indicate that wall temperatures of several thousand degrees could occur transiently with a quartz tube. Substantially lower temperatures (by factors of at least 5) would occur with an alumina tube. 
FRC formation in a conical theta-pinch and subsequent translation into a metal flux conserver of similar dimensions would be performed during the second year of the proposed research. A cone angle of about $4^{\circ}$ should be sufficient to provide the axial force required to accelerate the FRC out of the coil and to translate it into the flax conserver in less than $10 \mu \mathrm{s}$. The FRC will be trapped, either with an end mirror field $\alpha$ with shaping of the end portion of the flux conserver. The translated FRC confinement properties must be assessed inside the flux conserver, with an excluded flux array and laser interferometry. Numerical simulations with the MOQUI code will guide the experimental translation and trapping studies.

A key variable is the initial external field $B_{0}$ that is established inside the flux conserver prior to FRC translation. The external field controls to a great extent, the translation velocity, FRC trapping, translated FRC volume, and the ratio of transiated FRC separatrix radius to flux conserver radius. The FRC confinement and the onset of the $n=2$ rotational instability will be studied as function of $B_{0}$. The translation and trapping processes must be optimized, with flux conservers of various shapes, prior to liner implosion tests. Liner studies, performed in parallel with the above FRC formation studies, will contribute significantly to the definition of the flux conserver geometry.

c. Target Plasma Facillties. Target plasma formation will use the Los Alamos Coit facility. This facility includes a single Maxwell Shiva capacitor bank module, vacuum/load chamber, screen-room, "standard" diagnostic set, and data recording equipment. The capacitor bank is a two-stage Marx consisting of 126 $\mu \mathrm{F}$ capacitors in each stage. The maximum charge voltage is $60 \mathrm{kV}$ per stage, and when switched, erects to twice the charge voltage. Switching is provided by a Maxwell rail-gap switch. The maximum stored energy at full charge is $0.25 \mathrm{MJ}$. The total system inductance is roughly $100 \mathrm{nH}$, including the inductance of a typical load assembly. The bank can deliver (without too much stress) 2 MA of current rising in 2.5 $\mu s$. The output of the bank is cable-coupled to a vacuum/load chamber $(61 \mathrm{~cm}$ diameter by $80 \mathrm{~cm}$ long) by 40 RG-17/14 coaxial cables. At the load chamber, the power feed is converted to a generic coaxial feed (outer electrode is ground, inner electrode is positive). The specific experimental load assembly is bolted to the generic coaxial feed. This facility has previously been used for Dense-Plasma-Focus experiments, vacuum-power-flow experiments, plasma-flow-switch experiments, and z-pinch MTF target experiments. Removal of one experimental load assembly and installation of a different experimental load assembly is usually accomplished in 2-3 days.

In addition to the Maxwell Shiva module, the facility has ten FRX-C "compressor mocules" $(10 \mathrm{kV}, 50 \mathrm{~kJ}$ capacitor modules switched and crowbarred with ignitron switches). These modules are available for driving bias coils on a timescale longer than the liner $\mathrm{L} R$ time; perfect for FRC formation. One such module is already wired to a charging power supply and controls system, and is ready to drive a load.

The floor space of Colt is 2100 sq- $\mathrm{ft}$. There are 56 channels of high-speed signal recording. Present diagnostics include, drive-current Rogowski coils, B-dot probes with a minimum of 2-mm insertion into the plasma edge, 1 channel of $\mathrm{HeCd}(442 \mathrm{~nm})$ laser interferometer, visible framing camera, visible nearUV monochrometer, two 7-channel filtered soft-xray diode arrays. Thomson scattering is under (re) construction, using the previous LANL CTX $25 \mathrm{~J}$ pulsed ruby laser and multipoint spectrometer, and will be operational by the end of FY 1998.

The traditional FRC formation method requires a main-drive capacitor bank, a preionization bank, and several sets of bias coils powered by two independent capacitor banks. Thus, to produce FRC plasmas, the appropriate load structure must be designed and fabricated for installation in the Colt facility. An 
FRC-producing load assembly will require a parallel-plate feed driving a theta-pinch coil, coupled with a set of bias coils. In this case, we would disconnect the 40 output cables from the usual load-chamber and reconnect them to a short parallel-plate feed driving the theta-pinch coil. A separate vacuum chamber is required, but the existing pumping stand (roughing and turbo pumps) would be used.

\section{Liner}

a. Liner Goal. Develop the vacuum implosion of a $30-\mathrm{cm}$ long, 10-cm diameter, $1-\mathrm{mm}$ thick aluminum liner with a velocity of $3 \mathrm{~mm} / \mu \mathrm{s}$ and radial convergence of 10 .

b. Liner Approach. Typical liners used for DP applications have near-unity length to diameter ratios. FRC compression requires a length-to-diameter ratio of approximately 3. During the first two years, the Shiva-Star driver will be used to develop vacuum implosions of an FRC-relevant liner with length, width, and thickness dimensions of $30 \mathrm{~cm}, 10 \mathrm{~cm}$, and $1 \mathrm{~mm}$ respectively. Initial one-dimensional calculations (Fig. 4) indicate that Shiva-Star can drive this $250 \mathrm{~g}$ aluminum liner to the required implosion velocity of $3 \mathrm{~mm} / \mu \mathrm{s}$. In conjunction with prototyping the FRC liner, diagnostics will be fielded to measure impurity content in the liner compression zone. An initial assessment will be made of impurities associated with ejecta and glide-plane electrode interactions during vacuum implosions.

c. Liner Facilities (Shiva Star Overview). The experimental pulsed-power facilities at the Air Force Research Laboratory (Phillips Research Site, Kirtland AFB, NM) consist of: 1) two laboratory buildings with several capacitor banks ranging from tens of kilojoules, tens of kilovolts to 9 megajoules, 2) tens of megamps with direct discharge current risetimes of 1 to several microseconds; and 3) several moderately compact Marx banks with outputs in the few 100 kilovolt to megavolt, few ohm to tens of ohms, 100 nanosecond to microsecond range.

The 9 megajoule Shiva Star capacitor bank has a capacitance of 1300 microfarads, can be charged up to 120 kilovolts, can tolerate $75 \%$ voltage reversal, and has a bank plus transmission line inductance of 3 nanohenries. It is normally used with a series safety fuse that limits its current in fault mode to 40 megamps. Direct discharge currents in past experiments have ranged from 12-15 megamps to implosion loads to 30 megamps to inductive store-opening switch loads, with current rise times ranging from 3 to 10 microseconds, depending on load inductance. Plasma flow switches have been used to sharpen current rise times to 250 nanoseconds, delivering 10 megamps to implosion loads. Shiva Star has flexible charging and triggering options, enabling the use of portions of the bank for lower energy, faster risetime discharges, and the use of non-destructive loads. The Shiva Star bank also has a 0.75-megajoule auxiliary capacitor bank integrated into its structure. This auxiliary bank, called the plasma formation bank, has been used to form compact toroids for subsequent acceleration, and has been used to form plasma working fluid for subsequent compression by solid liner implosions. This auxiliary bank can be used to form a variety of initial plasma - magnetic field configurations for subsequent compression.

The AFRL facilities include radiofrequency shielded enclosures with mumerous fast transient digitizer and analog recorders for data acquisition. They include substantial vacuum and power supply hardware; pulsed current, voltage, and magnetic field diagnostics; rotating mirror and gated microchannel plate tube fast photography; optical, RF, vacuum-ultra-violet, X-ray, gamma, and neutron spectroscopy equipment; pulsed radiography equipment; fast closure shutters and shielding to protect and enable use of these diagnostics in the blast and debris environments encountered in higher energy (multi-megajoule) experiments. 
There are extensive complementary theoretical and computational abilities and resources. These include one-, two-, and three-dimensional radiation MHD and particle-in-cell codes which have been developed, and are being further developed, to guide and interpret experiments. There is extensive development of parallel versions of these codes, and of parallel processing, high performance computing techniques. A simple one-dimensional code that has proven useful for first-cut estimates has been used to examine the dynamics of the liner described above when driven by the Shiva Star main energy bank. The results are shown in Fig. 4.

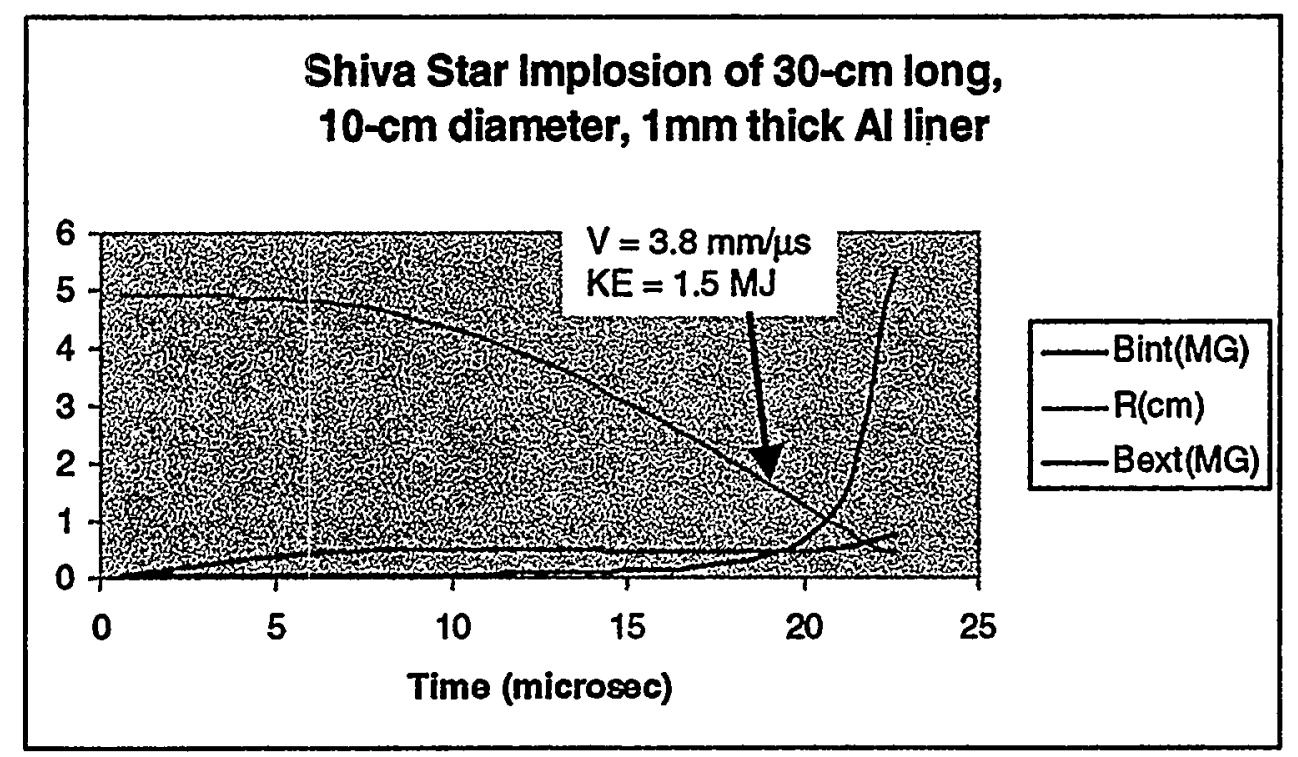

Fig. 4. One-dimensional simulation of liner implosion (AFRL) showing liner radius $R(t)$, external $B_{\theta}(M G)$ driving field, and internal $B_{z}$ vs. time.

\section{Integrated Compression}

a. Goal of Integrated Experiments. Demonstrate that substantial heating and increased $\mathrm{n} \tau$ results from liner implosion of an FRC according to present understanding of FRC physics.

b. Approach to Integrated Experiments. Cylindrical compression: The FRC will first be compressed radially in the third year by a near-cylindrical liner. We envisage this experimental campaign to use the Shiva-Star facility. For this case, the FRC would be in near 2-D elongated equilibrium during most of the compression, with $\mathrm{x}_{8}$ values anywhere from 0.4 to 0.9 by selecting initial liner bias field and radius. The latter can be done by proper timing of the FRC arrival into a liner in motion. Compressional (2.4-D) plasma heating can be achieved by cylindrical liner implosion because axial compression contributes an additional 0.4-D [Spencer83]. This option combines geometrical simplicity, considerable flexibility, and a high possibility of minimum impurity influx from the liner. In particular, the FRC separatrix during compression would quickly move away from the liner ends where metal vapor may result from liner/electrode interactions. In addition, the FRC separatrix divertor could also be more isolated from the inner liner radius, if thermal contact or impurities prove a major concern. Open-field-line FRC physics could be tested with low- $Z$ coatings on the inner liner surface. 
A complete set of FRC formation hardware will be procured and fabricated for such tests. FRC formation and translation will probably be performed with the liner in motion. The initial experiments will focus on proper timing and isolation of the different electrical circuits. The liner energy and radial velocity will be progressively increased, on successive shots, to estimate FRC heating and liner-plasma interactions for different compression ratios and ratios of separatrix to wall radii.

Development of plasma diagnostics to estimate FRC heating and confinement will be completed in time for integrated compression testing. The external magnetic field and the plasma density will be measured during plasma compression. Optical emission, end-on framing cameras, end-on inferometery, and neutron diagnostics will be available for the initial tests.

Quasi-spherical compression: Based on cylindrical compression results, the FRC could be compressed in nearly 3-D by a shaped liner. The FRC separatrix would remain close to the liner at all times during compression. This option has been explored theoretically [Alikhanov83] and experimentally [Es'kov81] to some extent. Recent 2-D MHD simulations with the MOQUI code [Milroy 82], shown in Fig. 5, indicate that an FRC target can be formed and moved in to a shaped liner in less than about $8 \mu \mathrm{s}$. The translated FRC could have values of $\mathrm{x}_{\mathrm{s}}$ as high as 0.9 inside the liner by choosing a small initial liner bias magnetic field. This option offers the potential for high liner compression efficiencies, for small liner volumes, for wall stabilization of possible instabilities such as rotational modes, and for quasi-spherical wall compression that maximizes plasma heating (full 3-D compression). Threats include possible FRC stability concerns due to small elongations and impurity influx from the liner. In particular, liner end effects need to be carefully evaluated. The decision to move forward with a quasi-spherical compression will be guided by the initial integrated compression experimental results and modeling of plasma-liner interactions.

c. Facilities for Integrated Experiments. This experimental campaign is envisaged to use the ShivaStar facility described above.

\section{Theory and Modeling Approach}

The theoretical effort, like the experimental effort, can be separated into three parts: (a) plasma formation, (b) liner implosion, and (c) liner-on-plasma compression. In each of these three areas, the effort can be broken into the following tasks:

(1) experimental design and parameter selection;

(2) detailed analysis and interpretation of experimental results;

(3) based upon task 2, evaluating the limitations of the computational models that formulating appropriate modifications;

(4) development and application of analytic theories to provide simple interpretations of experimental results;

(5) development of complex, highly non-linear models that are amenable only to numerical solution by incorporation into existing or yet-to-be-developed (e.g., ASCI) computer codes, and

(6) computationally exploring alternate configurations based on task 2.

Our starting point for computational modeling of MTF is the strongly coupled, highly non-linear equations of resistive, non-ideal $\mathrm{MHD}$. The most elemental system consists of a continuity equation, an equation of motion, an energy equation, and Faraday's law. The equation of motion includes the plasma self-pressure force and the Lorentz force and requires, for liners, a model of the material strength. The 
energy equation requires an equation-of-state and models of the thermal conductivity and radiative emission and includes, for liners, heating due to internal stresses. The simplest form of Ohm's law requires a resistivity model for magnetic field diffusion and requires the inclusion of Ohmic heating in the energy equation.

In general, the ion and electron temperatures can be different, in which case the single energy equation must be replaced by two energy equations that include the coupling of energy between the ions and electrons in addition to thermal conductivity, radiation (electrons), and Ohmic heating (electrons). Also, it may be necessary to include even a third energy equation that describes the temporal and spatial variation of the radiation energy density. In fact, most simulations of liners are "two-temperature" simulations where two energy equations are used, one to describe the material temperature and one to describe the radiation temperature.

For most plasma simulations, the transport coefficients (e.g., thermal conductivity, and resistivity) are "classical," i.e.; the prescriptions derived by Braginskii are used. However, the Braginskii presentation must be supplemented with models that incorporate partial ionization effects, because experience in modeling MTF-like plasmas has already shown that the traditional assumption of complete ionization, as an initial condition for a computation, is not satisfactory. In addition, experience in modeling the FRC has shown that it is necessary to invoke an "anomalous resistivity" to stimulate the experimentally observed field-line tearing and formation.

Some of the more successful FRC and other CT experiments have shown that standard MHD models do not adequately explain the plasma behavior, even when anomalous resistivity is included. Hence, we cannot discount the possibility that using available hybrid and kinetic codes may be required to adequately understand MTF plasma behavior. Unfortunately, these codes have not reached the level of development where they can be routinely used for experimental design, analysis, and interpretation (tasks $1,2,4)$ with the close correspondence between computation and experimental geometry and timescale expected from the MHD codes.

Existing models for material properties (e.g., equation-of-state, resistivity, strength) of candidate liner materials, such as aluminum and tungsten, have not always proven to be satisfactory. Although the properties at standard atmospheric conditions are well known, the temperature, density, and pressure of the liner material will change substantially during the course of the liner's acceleration and implosion, and the liner material will enter thermodynamic regimes for which the material properties have not been well characterized. We anticipate that many of the computational issues related to liners will have to be solved independently of this proposal in the ongoing Defense Program's liner research, but we also anticipate a need for MTF-specific research along these lines.

The preceding discussion should make it clear that using the available computational tools to perform the many "numerical experiments" needed to ensure the success of this proposal requires not only highly sophisticated understanding of the physical issues involved, but also a high level of empiricism and skills developed from "hands-on" experience. In conducting tasks 1-4, we will make use of the extensive expertise already developed in non-MTF contexts in the modeling of plasmas and liners.

Once experimental data becomes available from plasma formation experiments. the modeling focus will shift to task 2: analyzing the data and interpreting the experimental results. The detailed work here involves direct comparisons between observations and computations and, where necessary, resolving any 
discrepancies. Often, the computational analog requires a sophisticated "post-processor" computation. For example, the computational analog of a light-emission diagnostic requires not only the density and temperature profiles determined through the "computer experiment," but also calculations of emission and absorption of light integrated over the line of sight of the experimental diagnostic.

When discrepancies between simulations and observations are attributed to code deficiencies, modifications will be formulated (tasks 3,6 ). Because the ultimate goal is to move to liner-on-plasma experimentation as quickly as possible, the decision that a candidate plasma is "suitable for subsequent implosion" will most likely be made only after a close correlation is achieved between observations and simulations and after a projection (task 4) of attractive performance for a conceivable liner-on-plasma system.

In most simulations of the plasma formation stage, only the plasma behavior will be computed, and the walls surrounding the plasma or coils within the plasma will be represented as external or internal boundary conditions. Hence, in computational parlance, the plasma simulations are "single-material." However, the detailed simulation of liners and liner-on-plasma systems requires a "multi-material" approach. In a typical liner-on-plasma simulation, the plasma will be, of course, hydrogenic, but the liner will be metallic (e.g., alumimum) and the liner electrodes, or "glide planes," will be of higher density (e.g., stainless steel). The already existing capabilities to perform multi-material simulations enhance the prospects for rapid progress in all areas of MTF.

The theoretical and computational effort will begin by designing the FRC target plasma. Because the MOQUI code has already proven successful in this application, it will be the principal tool most exercised. An example of a recent MOQUI computation showing how an FRC can be formed in a density regime that is of interest to MIF is shown in Fig. 5. This example of work in progress assumes the Colt bank generates $5 \mathrm{~T}$ in 2.5 microseconds with a bias field of $1 \mathrm{~T}$ and initial fill pressure of $250 \mathrm{mT}$ (orr on the left hand conical theta pinch. The FRC is formed with $\mathrm{T}-250 \mathrm{eV}$, and $\mathrm{n}-2 \times 10^{17} \mathrm{~cm}^{-3}$. The geometry and liner bias fields are being iterated upon to develop a realistic liner shape that should be capable of trapping an FRC and imploding it using a variable-thickness liner shaped in a 5-cm region at each end near stationary electrodes. Flux imbedded in the liner on a slow time scale before FRC injection creates a magnetic well that centers the FRC in the liner. The idea is to achieve a uniform cylindrical implosion in the central $30 \mathrm{~cm}$ of the liner with electrical connection preserved by the deformed liner at the ends.

Because fully integrated liner-on-plasma multi-dimensional computations will be costly and manpower intensive, a hierarchy of computational procedures will be employed, beginning with simple zerodimensional system modeling and ending with the most complete, multidimensional integrated computation possible. An example of an intermediate computation is shown in Fig. 6. One-dimensional magnetohydrodynamic computations were done with the LANL code MHRDR, which has been previously benchmarked on a number of experiments in which the plasma density was in the MTF regime [Lindemuth 95]. 

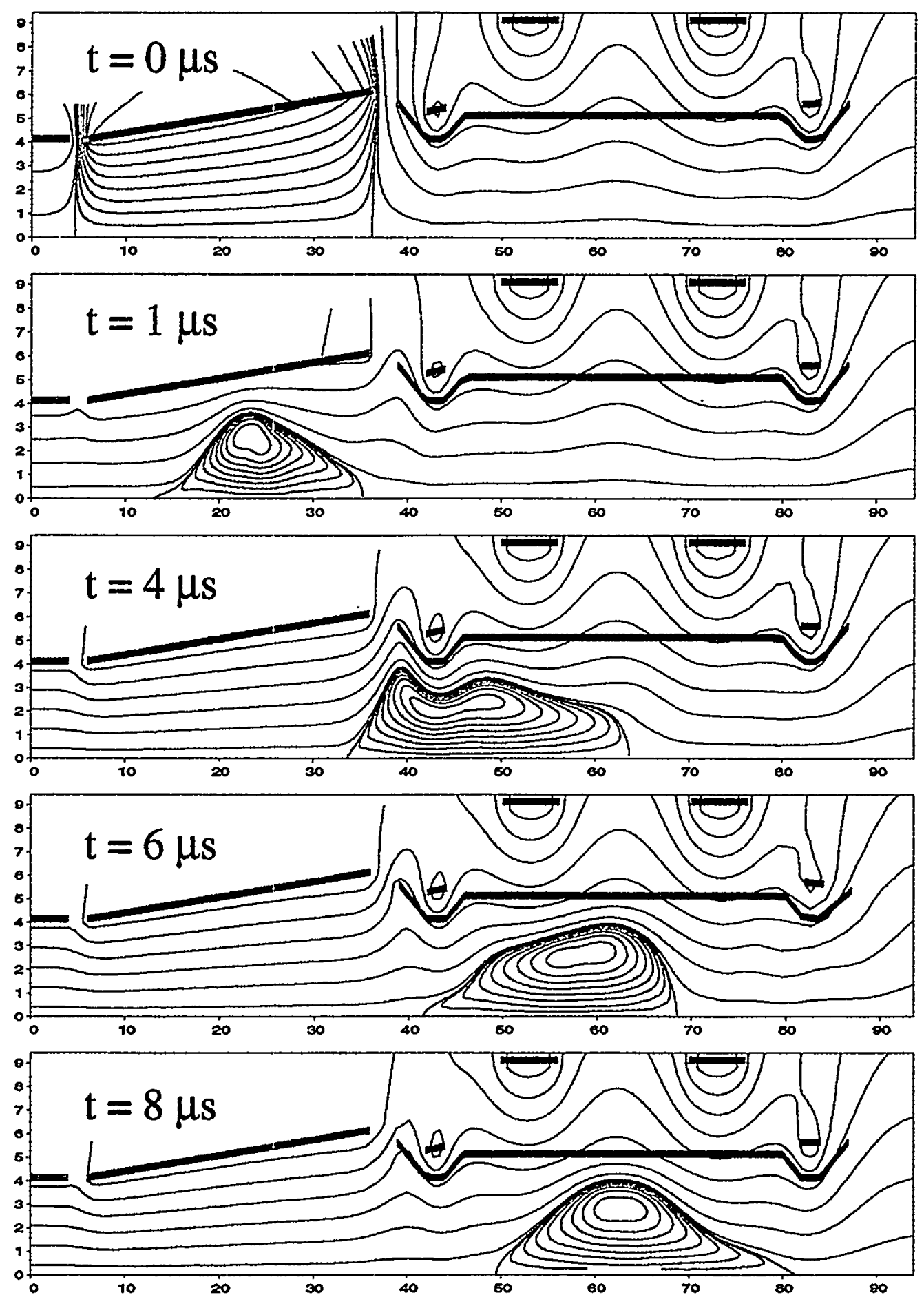

Fig. 5. MOQUI Calculation of FRC formation and injection into liner-compatible geometry. The conical theta pinch is on the left, and the liner is on the right. 

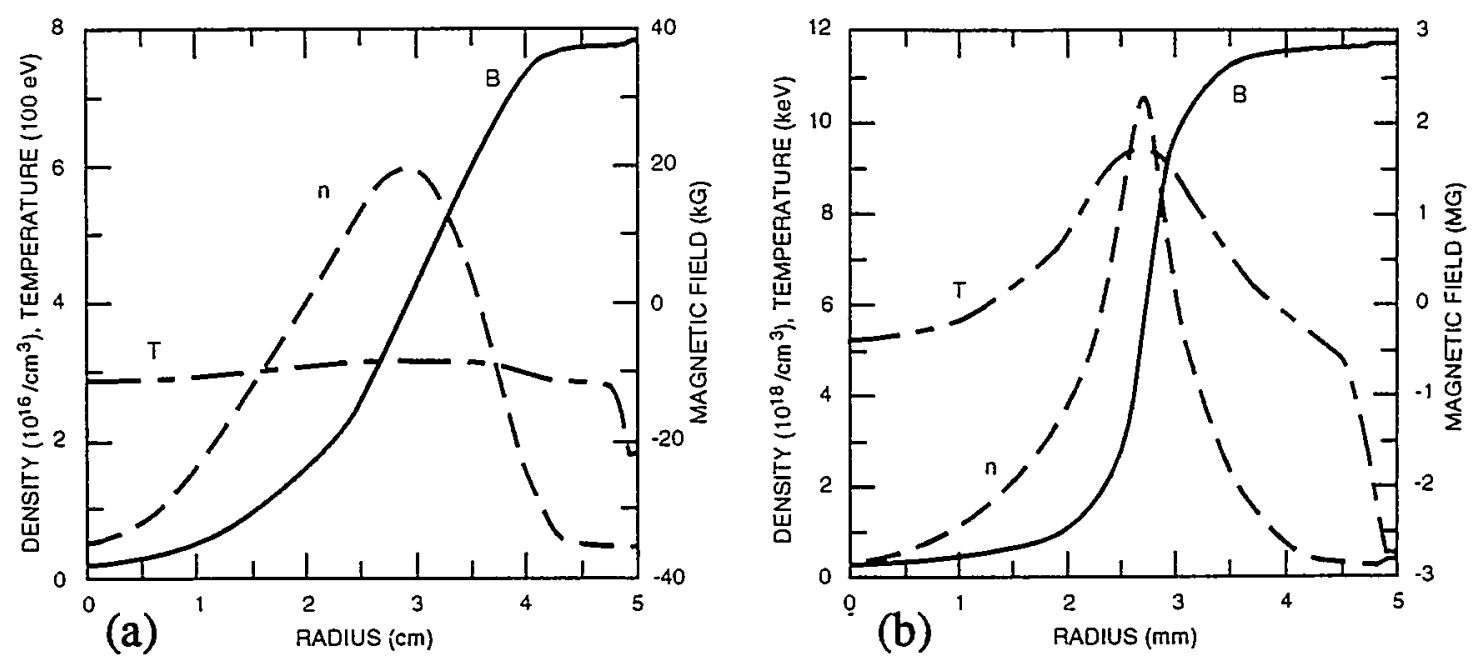

Fig. 6. FRC profiles of density (n), temperature (T), and poloidal magnetic field (B) at: (a) $t=2 \mu \mathrm{s}$, immediately prior to implosion at $3 \mathrm{~mm} / \mu \mathrm{s}$; (b) $t=17 \mu \mathrm{s}$, when liner has reached a radial convergence of 10 .

The purpose of the computations in Fig. 6 is to begin exploring the behavior of FRC plasmas under liner implosion conditions that result from a nearby low-temperature liner boundary. The computations were initialized at $\mathrm{t}=0$ with radial profiles of density, temperature, and magnetic field representative of those in an FRC immediately after injection into a liner. The model is only one-dimensional, which makes the poor approximation that all field lines are infinitely long. Thus there is no distinction for transport in the axial direction inside and outside the separatrix. Classical perpendicular transport in the radial direction ("classical" Braginskii) is included in the computations under the assumption that the wall is at zero temperature. Resistive diffusion, Ohmic heating, and Bremsstrahlung radiation were also included in the computations. Other than demonstrating an example of code capability, the calculation demonstrates mainly that classical cross-field transport is negligible even for the very small dimensions and mediumhigh density that might occur between an FRC separatrix and the nearby liner wall.

Some details of the calculation are interesting to consider. The initial profiles were allowed to relax for $2 \mu \mathrm{s}$, leading to the profiles shown in Fig. 6 . At $2 \mu \mathrm{s}$, the outer wall was computationally moved radially inward at a velocity of $3 \mathrm{~mm} / \mu \mathrm{s}$, thereby simulating the compression of the FRC plasma by a cylindrical liner. At $17 \mu \mathrm{s}$, the time at which the liner stops at a radius of $5 \mathrm{~mm}$ and a convergence of 10 , the profiles had evolved into those shown in Fig. 6 . An adiabatic compression would have led to a density increase of $100\left(10^{2}\right)$, a magnetic field increase of $100\left(10^{2}\right)$, and a temperature increase of $22\left(10^{4 / 3}\right)$. The profiles show approximately adiabatic behavior. However, the adiabaticity is affected in part by the thermal conduction and resistive diffusion, and the profiles show some steepening because the magnetic field under compression conditions is "stiffer" than the plasma, with the field having an effective $\gamma$ of 2 , compared to the plasma $\gamma$ of 5/3. These computations illustrate a basic difference between MTF and ICF. Whereas ICF requires strong, carefully timed shocks to raise the fuel to fusion temperatures, MTF is essentially a slow, adiabatic process that is relatively insensitive to the acceleration profile of the liner. 
5. Schedule, Key Decisions, and Budget

Figure 7 shows in pert-chart form the main tasks and key decision points.

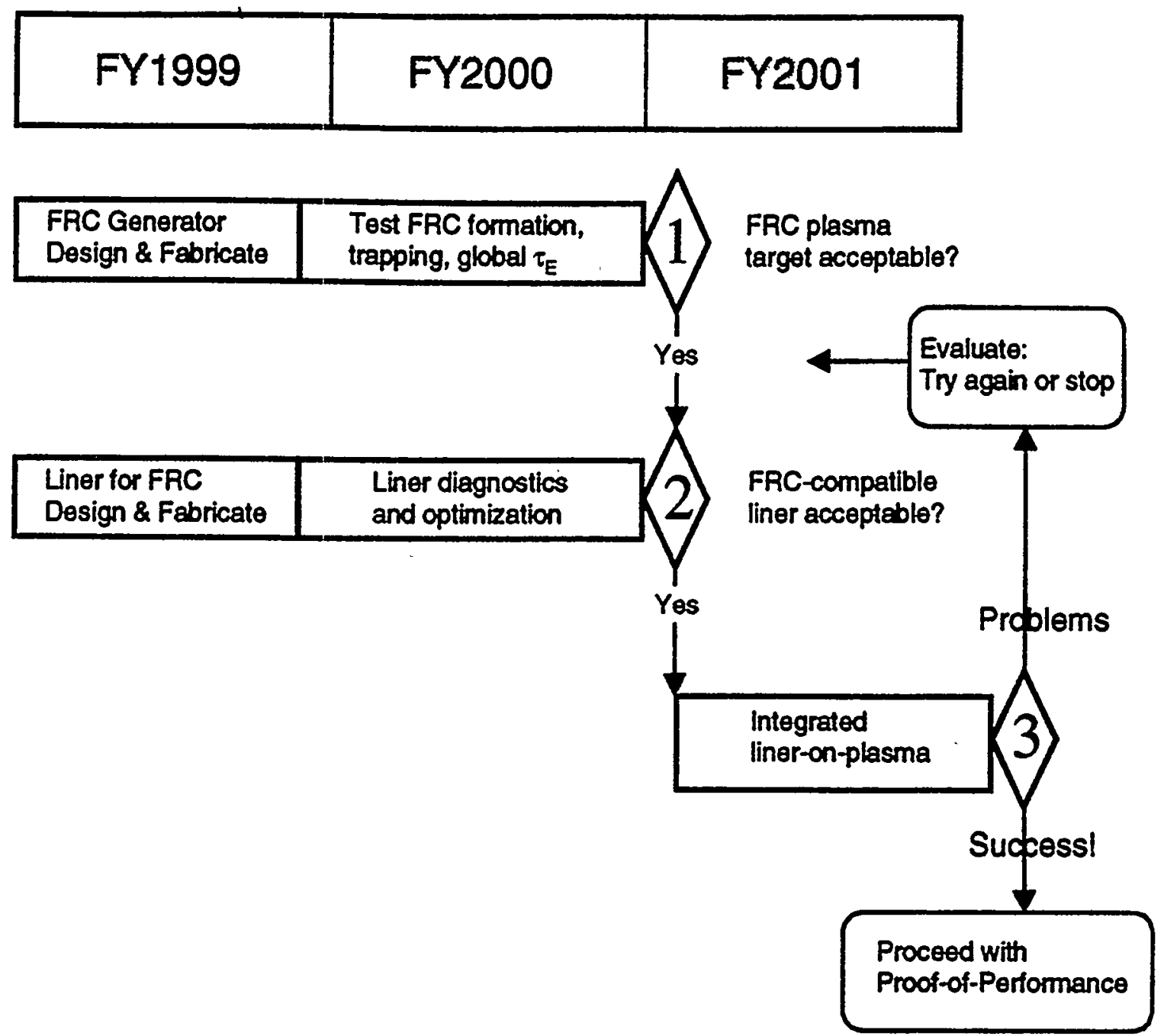

Fig. 7. MTF Schedule and logic diagram for tasks and key decisions 1,2, and 3.

The three key decisions are critical points in time when information is available to decide on how to proceed. See Table 4 for a detailed discussion of criteria for success. For decisions 1 and 2 at the end of the second year, it is understood that problems may require a reevaluation and further iterations before proceeding to integrated liner-on-plasma experiments. If 1 and 2 are favorable, then a 1-year campaign of integrated liner-on-plasma experiments will be carried out. At the end of that time a careful evaluation is called for to evaluate MTF and to decide whether or not to proceed with Proof-of-Performance. In the same time frame the ATLAS pulsed-power facility will become operational at Los Alamos, which would make a Proof-of-Performance program cost effective as well. 
Table 4. Major tasks, milestones, and key decision points.

\begin{tabular}{|c|c|c|}
\hline Start Date & Task, milestone, or decision & Description \\
\hline $\begin{array}{l}\text { FY99Q1 } \\
\text { (yr and } \\
\text { quarter) }\end{array}$ & $\begin{array}{l}\text { Task: Design and Fabricate FRC } \\
\text { Target Plasma Hardware }\end{array}$ & $\begin{array}{l}\text { Reconfigure LANL Colt bank and diagnostics } \\
\text { for FRC experiments. Design and order vacuum } \\
\text { components, quartz tubes, conical theta pinch } \\
\text { coil, and dummy liner. }\end{array}$ \\
\hline FY99Q1 & $\begin{array}{l}\text { Task: Design and Fabricate Liner for } \\
\text { FRC targets }\end{array}$ & $\begin{array}{l}\text { Use 2D numerical simulation to obtain detailed } \\
\text { design of shaped liner. Prepare diagnostic } \\
\text { system for liner implosion tests on Shiva Star. }\end{array}$ \\
\hline FY99Q4 & $\begin{array}{l}\text { Milestone: Form first FRC plasma in } \\
\text { liner-compatible geometry. }\end{array}$ & $\begin{array}{l}\text { Minimum initial diagnostics are diamagnetic } \\
\text { loop, axial probe array, and one chord of side- } \\
\text { on interferometry. }\end{array}$ \\
\hline FY99Q4 & $\begin{array}{l}\text { Milestone: Implode first FRC. } \\
\text { compatible liner }\end{array}$ & $\begin{array}{l}\text { Minimum initial diagnostics in addition to } \\
\text { current waveform are magnetic probes, } 3 \text {-axis } \\
\text { radiography, and axial framing photography. }\end{array}$ \\
\hline FYOOQ1 & $\begin{array}{l}\text { Task: Test FRC formation, translation, } \\
\text { trapping, and global energy } \\
\text { confinement. }\end{array}$ & $\begin{array}{l}\text { Additional diagnostics include axial } \\
\text { interferometry, single-point Thomson scattering, } \\
\text { bolometery, impurity spectroscopy, and neutron } \\
\text { diagnostics. }\end{array}$ \\
\hline FY00Q1 & $\begin{array}{l}\text { Task: Liner diagnostics and } \\
\text { optimization }\end{array}$ & $\begin{array}{l}\text { Additional diagnostics include pin arrays, } \\
\text { pressure transcucers and spectroscopy to detect } \\
\text { metal vapor near electrodes, and injected } \\
\text { magnetized plasma for development of xray } \\
\text { impurity diagnostics. }\end{array}$ \\
\hline FYOOQ4 & $\begin{array}{l}\text { Key Decision: Is the FRC plasma } \\
\text { target acceptable for integrated liner- } \\
\text { on-plasma experiments? }\end{array}$ & $\begin{array}{l}\text { Criteria: No signs of gross instability; After } \\
\text { FRC is centered in liner: Density } \sim 10^{17} \mathrm{~cm}^{-3}, \mathrm{~T} \\
\sim 300 \mathrm{eV}, \tau_{\mathrm{E}} \sim 10 \mu \mathrm{s} \text {. }\end{array}$ \\
\hline FYOO & $\begin{array}{l}\text { Key Decision: Does FRC-compatible } \\
\text { liner implosion appear acceptable? }\end{array}$ & $\begin{array}{l}\text { Criteria: Reasonably symmetric cylindrical } \\
\text { convergence of } 10: 1, \mathrm{~B}_{\max } \sim 5 \mathrm{MG} \text {, central } \\
\text { volume (away from electrodes) free of metal } \\
\text { vapor. }\end{array}$ \\
\hline FY01Q1 & $\begin{array}{l}\text { Task: Integrated Liner-on-Plasma } \\
\text { Experiments }\end{array}$ & $\begin{array}{l}\text { Install FRC system and diagnostics on Shiva } \\
\text { Star at AFRL. Conduct campaign of } \\
\text { approximately } 20 \text { high-energy liner-on-plasma } \\
\text { shots. }\end{array}$ \\
\hline FY01Q4 & $\begin{array}{l}\text { Key Decision: Is the MTF Proof-of- } \\
\text { Principle adequately demonstrated? }\end{array}$ & $\begin{array}{l}\text { Criteria: Computer simulations in agreement } \\
\text { with plasma parameters as given in Table } 1 \text {, } \\
\text { thus providing a basis for proceeding to Proof- } \\
\text { of-Performance }\end{array}$ \\
\hline
\end{tabular}

The budgets for this work will be detailed in normal Field Work Proposals and similar documentation provided by the various team institutions. Table 5 summarizes the main components of the budget with a breakdown for the two largest budget categories of experimental activities at AFRL and LANL. The reason the funding level can be so low despite the ambitious objectives just described is the considerable 
leveraging of existing competencies and facilities that have been created by Defense Programs (DOE) and the Air Force (DOD). Another important aspect that keeps the cost low, but is hard to quantify, is that the proposed support is in many cases for part-time involvement of the participants. In effect, this project will only be paying for what is needed to do the MTF work, and the balance of people's time will be supported by other projects, some related, and some not. We propose to begin this initial Proof-ofPrinciple examination of MTF without creating a "stand-alone" organization with the implied commitment for ongoing funds.

Table 5. Major budget components (\$K)

\begin{tabular}{|c|c|c|c|c|}
\hline Institution & Item & FY99 & FYOO & FY01 \\
\hline \multirow{8}{*}{ AFRL } & Experiments & & & \\
\hline & Salaries and Fringe & 300 & 300 & 300 \\
\hline & Hardware and consumables & 400 & 375 & 375 \\
\hline & Diagnostics & 25 & 50 & 50 \\
\hline & Travel, publications, etc. & 25 & 25 & 25 \\
\hline & Total Direct & 750 & 750 & 750 \\
\hline & Indirect & 250 & 250 & 250 \\
\hline & AFRL Subtotal & 1000 & 1000 & 1000 \\
\hline \multirow[t]{9}{*}{ LANL } & Salaries and Fringe & 1000 & 1000 & 1000 \\
\hline & Hardware and consumables & 400 & 200 & 200 \\
\hline & Diagnostics & 100 & 100 & 100 \\
\hline & Travel, publications, etc. & 100 & 100 & 100 \\
\hline & Total Direct & 1600 & 1600 & 1600 \\
\hline & Indirect & 1400 & 1400 & 1400 \\
\hline & LANL Subtotal & 3000 & 3000 & 3000 \\
\hline & Experiments Total & 4000 & 4000 & 4000 \\
\hline & Theory \& Computing & & & \\
\hline GA & Wall-plasma interactions & 100 & 100 & 100 \\
\hline LANL & Plasma and liner modeling & 1000 & 1000 & 1000 \\
\hline LLNL & Plasma theory and modeling & 300 & 300 & 300 \\
\hline \multirow[t]{2}{*}{ U. Washington } & FRC Formation \& Translation & 100 & 100 & 100 \\
\hline & $\begin{array}{l}\text { Theory \& Computing Total } \\
\text { Energy Systems Scoping Studies }\end{array}$ & 1500 & 1500 & 1500 \\
\hline LANL & Pulsed-system perspective & 100 & 100 & 100 \\
\hline LLNL & ICF and liquid-wall perspective & 100 & 100 & 100 \\
\hline Westinghouse & Team leader of activity & 300 & 300 & 300 \\
\hline \multirow{3}{*}{ Undetermined } & Energy Scoping Studies Total & 500 & 500 & 500 \\
\hline & Supporting Exploratory Research & 600 & 600 & 600 \\
\hline & Grand Total & 6600 & 6600 & 6600 \\
\hline
\end{tabular}

An unusual aspect of budgeting is the cost for equipment destroyed in high-energy liner implosions. Fortunately, the apparatus needed for FRC formation, while relatively complicated, is fairly small in dimension (see Fig. 8) and cost. The hardware cost per assembly unit is estimated at $\$ 25 \mathrm{~K}$ based on experience with systems of similar-size. 


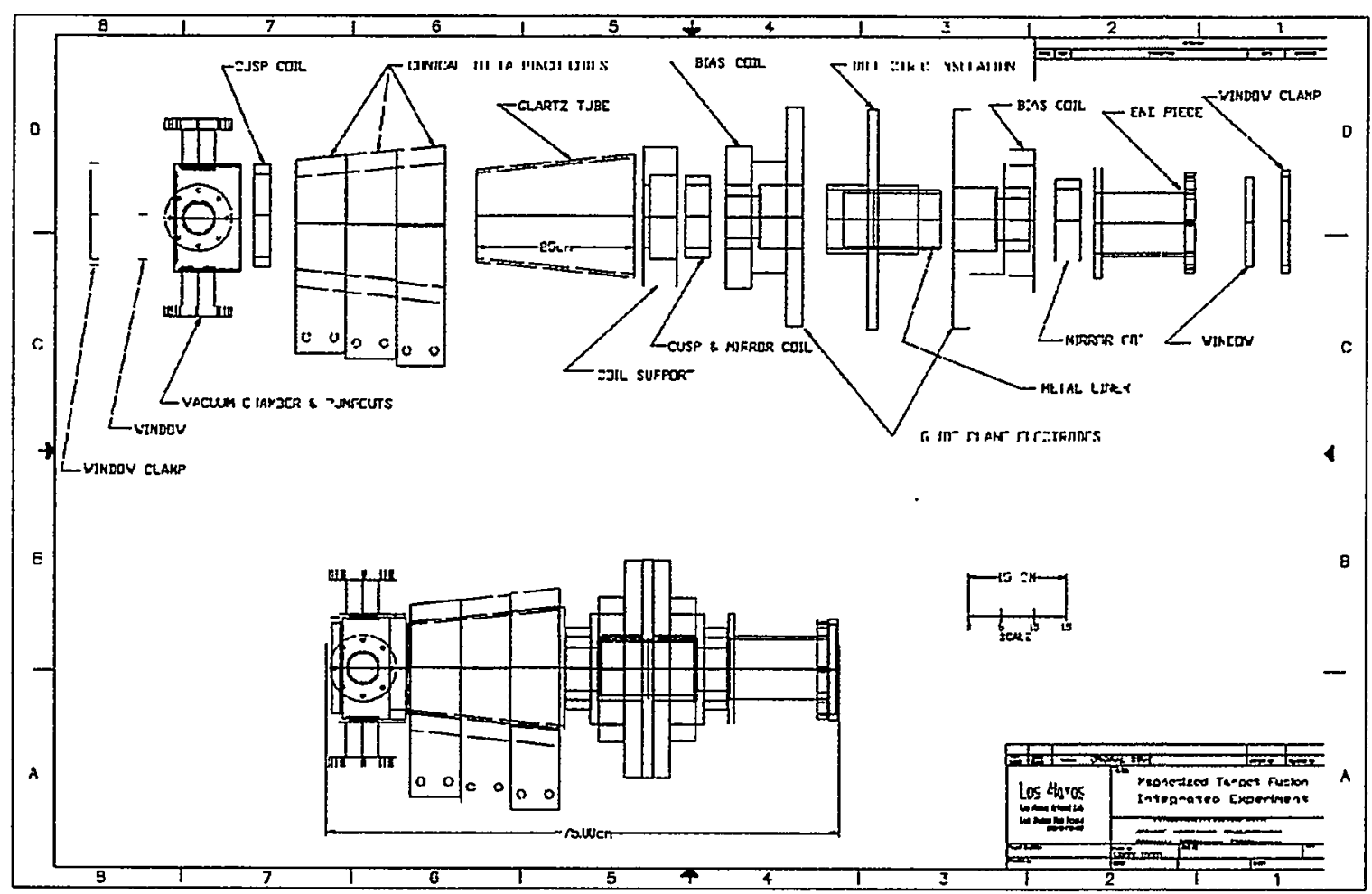

Fig. 8. Conceptual layout of an FRC plasma source mated to a liner assembly. Both the line drawing (above) and 3D view (below) show an expanded view and an assembled view.
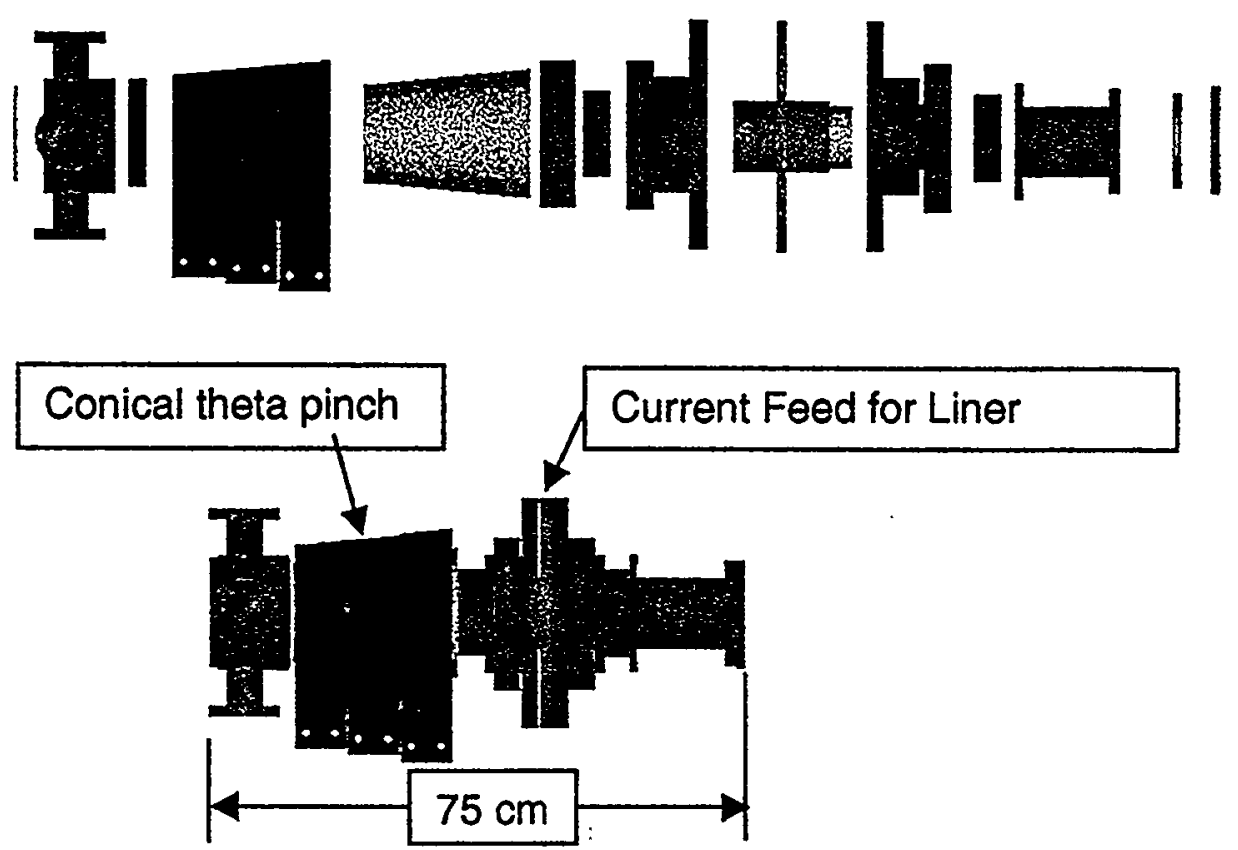


\section{PART D: POTENTIAL FOR PRACTICAL FUSION POWER}

While reactor considerations are not central to this proposal, it is important that some effort be devoted to this task. Conceptual-level reactor studies, conducted in parallel with basic scientific programs, have two primary objectives. The first is to identify key technological issues requiring further research and development. The second is to provide ongoing assurance that the end product being worked toward (i.e. an electricity producing power plant) is both economically and environmentally attractive. Both of these require close communication and iteration between researchers and designers as concepts are refined and improved, issues are resolved and new ones arise. The end-product concept is also needed to define a meaningful development path. In this process, the experience of industry in taking a new energy technology from conceptual design to commercial application provides an important perspective. For Westinghouse, this has most recently included fission reactors (water and liquid metal), advanced gas turbines, solid oxide fuel cells, thermoelectrics, and photovoltaics

An early MTF reactor study [Moses79] identified two key technology feasibility issues: (1) material selection and mechanical design of the leads/liner structure, including recovery and recycling of the materials; and (2) pulse power requirements, which were beyond the state-of-the-art at the time of the study. While these were based upon a specific point design, they are generic issues that must be addressed by any MTF reactor concept.

Plasma conditions for MTF are generally described as midway between those of magnetic and inertial fusion systems (in terms of plasma density and time scales), but are much closer to Inertial Fusion Energy (IFE) as a reactor embodiment. Both are inherently pulsed systerns, and offer the same potential for liquid wall protection schemes with regard to radiation damage and high heat flux removal. IFE issues of chamber beam transport and targeting are traded against MTF issues of power-feed and pulse containment. Both have key feasibility issues requiring very different solutions. The IFE driver (laser or particle beam) analogue is the pulsed power system required to drive the liner implosion. In this case, the much lower power requirements of MTF offer the possibility of more "conventional" low-cost power sources.

While similar in some respects, there are important differences between IFE and MTF for reactor design. These include pulse rep rate and energy output/pulse (Fig. 9). Typical values for an IFE system are 5-10 $\mathrm{Hz}$ and 300-500 MJ/pulse for a $1000 \mathrm{MWe}$ reference plant, with 5-10 MJ on target. In contrast, MTF will might work with outputs in the $10 \mathrm{GJ}$ range with rep rates less that $1 \mathrm{~Hz}$. Energy input to the liner depends upon the extent of the burn, and hence the energy multiplication. However, 100's of MJ may be required, making the pulse power system a challenge.

These similarities and differences in requirements between IFE and MTF present new opportunities, as well as new challenges, in power plant design. The similarities permit us to make use of the extensive design studies that have been performed over the past 20 years as starting points for an MTF design, particularly liquid-wall protection schemes. Differences can be exploited. For example, one of the limitations in the design of the IFE chamber was related to clearing debris and re-establishing stable liquid jet flow between shots. With a simple gravity flow arrangement, the rep rate was limited to be at least $1 \mathrm{~Hz}$ [Monsler78]. In order to overcome this limitation, a complex oscillating jet flow system was adopted [Moir94]. With the MTF rep rate larger than $1 \mathrm{~Hz}$, we can revert to the simpler concept. 


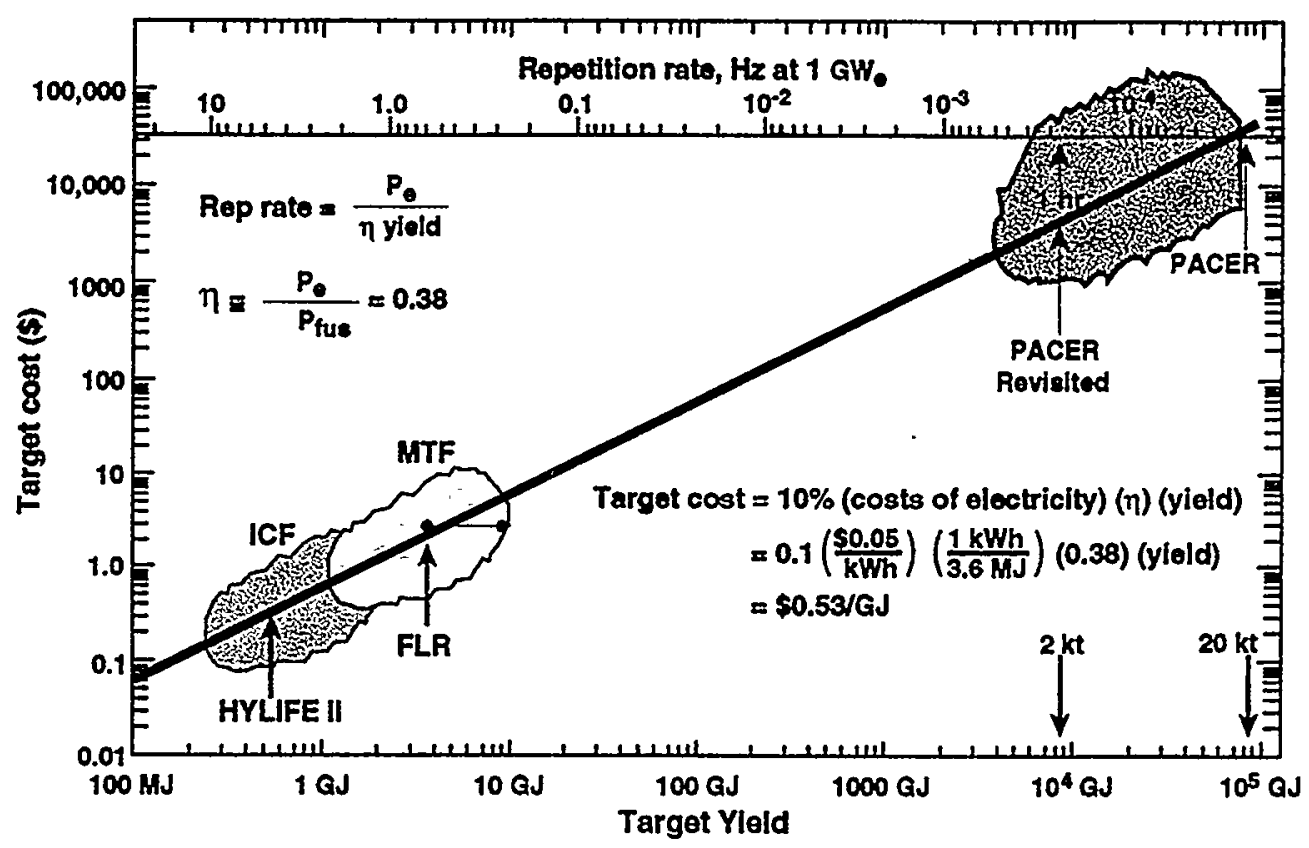

Fig. 9. Allowable expense of consumables per pulse vs. target yield in Joules.

For these large-yield, low-cycle shots we can also make use of the work done in examining nuclear explosives as an energy source [Hubbard74]. The most recent embodiment of this concept, Pacer Revisited [Cal190] utilizes an underground steel-lined cavity with a simple vertical array of thin (2-mm diameter) molten salt (flibe) jets for wall protection and heat removal. In our case, we would substitute plasma/liner/electrodes for bomb insertion. For a 2 Kton device ( $8 \mathrm{TJ}$ ), a chamber volume of 250,000 cubic meters was required. Scaling by yield, a $10 \mathrm{GJ}$ chamber might have a volume of about 300 cubic meters (a 4-meter radius sphere). By way of comparison, the ITER vacuum vessel has a volume of about 1500 cubic meters.

Large pulses of energy (5-80 GJ), are also ideally suited to another power conversion concept, that of MHD power generation as proposed originally by Velikov and studied recently by Logan [Logan93]. In this approach, the large fusion pulse is used to flash vaporize a solid, nearly spherical blanket surrounding the leads/liner structure. The blanket then becomes the working fluid, at about $1 \mathrm{ev}(12,000 \mathrm{~K})$ temperature and 100 bar pressure. A quasi-steady-state source of plasma for MHD energy conversion is maintained by a several-shot plasma inventory in the reactor cavity. While a high MHD energy conversion is obtained, the overall conversion efficiency is low, partially because of high heat rejection of the working fluid. This could be improved with the addition of a steam bottoming cycle. Compared to the "conventional" liquid wall/steam cycle energy conversion systems above, this concept also raises many new issues with regard to the MHD channel, as well as the high chamber wall operating temperature $(2500 \mathrm{~K})$. However, this concept has the extremely interesting appeal of doing power conversion without a conventional steam cycle and the associated balance of plant costs. Clearly it deserves attention as a design option for an advanced system. 
Inherent to any pulserisystem is the need for some form of thermal or electrical energy storage so that a nearly constant power output can be supplied to the grid. The problem becomes more challenging as the pulse power goes up and the rep rate goes down. In most cases it can be handled in a cost-effective manner by having the coolant inventory in the primary system high enough to maintain (nearly) constant temperatures across the primary heat exchanger. At some point, the inventory of coolant may become sufficiently high to impact cost and safety and more detailed designs are needed to resolve the issue. The MHD system, as described above, deals with this problem by maintaining an inventory of plasma in the reaction chamber sufficient to maintain a constant pressure and temperature input to the MHD channel.

Another key element, if the MTF concept is to become a viable energy source, is the development of a suitable pulse power system, including pulse proctuction, storage, conditioning, and switching. As discussed above, earlier studies indicated the requirements were beyond the state-of-the-art at the time. These studies focused on the homopolar generator/inductive storage and purely capacitive energy storage options. Developments since that time in pulsed AC machines, Superconducting Magnetic Energy Storage Systems (SMES), and switching warrants investigation of these technologies. Advances have also been made in the earlier systems considered. A study is needed to reexamine this issue, focusing particularly on the technical feasibility, risk, and cost of rotating machine, capacitor bank, and SMESbased pulse power systems. Solid state switching options should also be included. Designs should be compared in a trade-off analysis and the best options selected. Even by present standards, the very high voltage (up to $200 \mathrm{kV}$ ) and extremely high current (up to $250 \mathrm{MA}$ ) required for a reactor represent a major design challenge. 


\section{PART E: COMMUNITY R AND D PLAN}

In the past few years, a growing number of researchers have been attracted to high-density pulsed magneto-inertial fusion (MIF) approaches. The term MIF is used to represent the all-inclusive set of pulsed high-pressure (inertially confined) approaches to fusion that involve magnetic field in an essential way. MTF is the subset that involves an imploding liner for pdV heating and magnetic field for suppression of thermal conduction.

By means of numerous professional meetings and personal interactions, a general consensus has emerged that a PoP experiment using liners to compress a $C T$ is the desirable first step. In response to an OFES request, a community-based R\&D Roadmap (URL http./fusionenergy.lanl.gov/R\&DRoadmap.pdf) was prepared and presented at the April 1998 Innovative Confinement Concepts meeting at Princeton.

Collaborations important for success of this MTF PoP proposal include a broad-based scientific exchange going well beyond the specific collaborations and deliverables of this PoP program. The growing MIF community includes scientists in various universities such as UC Irvine, U. Nevada, Reno, UC Berkeley, and researchers abroad including Russia, France, and New Zealand. A large closely linked group is the ongoing compact toroid research at the Univ. of Washington, LLNL, PPPL, Univ. of Texas, UC Berkeley, Osaka University, Univ. of Tokyo, and elsewhere. These scientists provide an important source of intellectual vitality and peer review. In addition, there is the pulsed-power community with its various specialties such as high-pressure hydro studies, pulsed high-magnetic fields, and fast $\mathrm{Z}$ pinches for procucing $x$-ray radiation (most notably at SNL).

A national Council for MIF, patterned after the organization of NSTX, is proposed to assist OFES in guidance of this program. The Council, appointed by OFES, would request and review proposals for the "undetermined" Exploratory Concept funding of Table 5, and this advice would be forwarded to OFES. There is a strong feeling in the MIF community that continued exploratory work is required if MTF or MIF are to succeed. For MTF the physics of wall-plasma interactions, the variety of plasma targets, options for composite liner materials, and so forth, all suggest that a growing program will be needed for a Proof-of-Performance step, If this proposed Proof-of-Principle program is successful, such an effort would be readily justified. 


\section{PART F: LITERATURE CITED}

[Alikhanov83] S.G. Alikhanov, V.P. Bakhtin, A.G. Es'kov et al., "Three-dimensional plasma compression in a Z-pinch liner system-Transport and compression of a compact torus by a quasi-spherical liner," in Plasma Phys. and Controlled Nuclear Fusion Research 1982, Vol.3, IAEA, Vienna (1983), 319.

[Armstrong87] W.T. Armstrong and J.A. Morgan, "Liner compression of a magnetically confined FRC plasma," Proceedings of the 4th International Conference on Megagauss Magnetic Field Generation and related Topics (Santa Fe, 1986), Plenum Press, NY (1987) p. 683.

[Barns74] J. F. Barns, P. J. Blewett, R. G. McQueen, K. A. Meyer, and D. Venable, "Taylor Instability in Solids," J. of Appl. Phys. 45, 2 (1974).

[Barnes79] D. C. Barnes, C. E. Seyler, D. V. Anderson, in Compact Toruses and Energetic Particle Injection (Proc. US-Japan Joint Symp., Princeton, NJ, 1979), Princeton Plasma Physics Laboratory, Princeton University (1979), 110.

[Barnes86] D. C. Barnes, J. L. Schwarzmeier, H. R. Lewis, and C. E. Seyler, "Kinetic tilting stability of field-reversed configurations," Phys. Fluids 29 (8), 2616-2629 (1986).

[Barnes97] D. C. Barnes, "Scaling Relations for High-Gain, Magnetized Target Fusion Systems," Comments on Plasma Phys. and Controlled Fusion 18(3) (1997).

[Cal190] C. J. Call and R. W. Moir, “A Novel Fusion Power Concept Based on Molten-Salt Technology: PACER Revisited," Nucl. Science and Engineering 104, 364-373 (1990).

[Carlsón87] A. W. Carlson, "Search for lower-hybrid-drift fluctuations in a field-reversed configuration using $\mathrm{CO}_{2}$ heterodyne scattering," Phys. Fluids 30 (5), 1497-1509 (1987).

[Chandrasekhar61] S. Chandrasekhar, "Hydrodynamic and Hydromagnetic Stability," Oxford University Press, London, 248 (1961).

[Drake96] R. P. Drake, J. H. Hammer, C. W. Hartman, L. J. Perkins, and D. D. Ryutov, "Submegejoule Liner Implosion of a Closed Field Line Configuration," Fusion Tech. 30, 310-325 (1996).

[Drucker80] D. C. Drucker, "Taylor Instability of the Surface of an Elastic-Plastic Plate," Mechanics Today 5, Paragamon Press, $27-47$ (1980).

[Es'kov81] A.G. Es'kov, M.I. Kitaev, R.Kh. Kurtmullaev et al., "Experiments in the Tor-Liner device", in Controlled Fusion and Plasma Physics (Proc. 10th European Conference, Moscow, 1981) paper L-5.

[Es'kov83] A.G. Es'kov, N.P. Kozlov, R.Kh. Kurtmullaev et al., "Energy balance in a system with quasispherical liner compression", Sov. Tech. Phys. Lett. 9, 16 (1983).

[Faeh197] R. J. Faehl, P. T. Sheehey, R. E. Reinovsky, I. R. Lindemuth, A. M. Buyko, V. K. Chernyshev, S. F. Garanin, V. N. Mokhov, V. B. Yakubov, "Modeling and Analysis of the High Energy Liner Experiment, HEL," in Digest of Technical Papers: 11th IEEE International Pulsed Power Conference, edited by G. Cooperstein and I. Vitkovitsky (IEEE, Piscataway NJ, 1997), p. 1375.

[Feinberg76] B. Feinberg, "Experimental study of hot plasma in contact with a cold wall," Plasma Physics 18 (4) 265-275 (1976). 
[Gerwin79] R. A. Gerwin and R. C. Malone, "Adiabatic Plasma Heating and Fusion-Energy Production by a Compressible Fast Liner," Nucl. Fusion 19(2), 155-177 (1979).

[Gross75] R. A. Gross, "Physics of a wall-confined fusion system," Nuc. Fus. 15, 729 (1975).

[Hagenson81] R. L. Hagenson and R. A. Krakowski, "A Compact Toroid Fusion Reactor Based on the Reversed Field $\theta$-Pinch, Los Alamos Scientific Laboratory report LA-8758-MS (1981).

[Hamasaki79] S. Hamasaki and N.A. Krall, "Anomalous diffusion calculations in field-reversed configurations," IEEE Conf. record, Montreal 1979, p.143.

[Hoffman86] A. L. Hoffman, R. D. Milroy, J. T. Slough, and L. C. Steinhauer, Formation of fieldreversed configurations using scalable, low-voltage technology," Fusion Technology 9 (1) 48 (1986).

[Hoffman93a] A. L. Hoffman et.al., "The Large-s FRC Experiment (LSX)," Fusion Technology 23 (2), 185-207 (1993).

[Hoffman93b] A.L. Hoffman and J.T. Slough, "Field-reversed configuration lifetime scaling based on measurements from the Large s Experiment," Nuclear Fusion 33,(1), 27-38 (1993).

[Horton94] W. Horton, "Chaos and structures in the magnetosphere," Physics Reports 283, 265 (1997).

[Hubbard74] H. W. Hubbard, R. P. Hammond, "PACER: A practical fusion power concept," Eleventh intersociety energy conversion engineering conference (A.I.C.E., New York, 1976).

[Ishida92] A. Ishida, L. C. Steinhauer, and R. Kanno, "Tilt stability of a gyroviscous field-reversed configuration with realistic equilibria," Phys. Fluids B 4 (5), 1280-1286 (1992).

[Kirkpatrick91] R. C. Kirkpatrick and I. R. Lindemuth, "Ignition and burn in inertially confined magnetized fuel," Fus. Technology 20, 834 (1991).

[Lebedev93] A. I. Lebedev, P. N. Nisovtsev, and V. A. Rayevsky, "Rayleigh-Taylor Instability in Solids," Proc. $4^{\text {th }}$ Intl. Workshop on the Physics of Compressible Turbulent Mixing, Cambridge, England (March 1993).

[Lindemuth78] I. R. Lindemuth, J. S. Pettibone, J. C. Stevens, R. C. Harding, D. M. Kraybill, and L. J. Suter, "Unstable Behavior of Hot, Magnetized Plasma in Contact With a Cold Wall," Phys. Fluids 21(4), April 1981.

[Lindemuth81] I. R. Lindemuth and M. M. Widner, "Magnetohydrodynamic behavior of thermonuclear fuel in a preconditioned electron beam imploded target," Phys. Fluids 24, 746 (1981).

[Lindemuth91] I. R. Lindemuth and R. C. Kirkpatrick, "The Promise of Magnetized Fuel: High Gain in Inertial Confinement Fusion," Fusion Technology 20 (December 1991).

[Lindemuth95] I. R. Lindemuth, et al., "Target Plasma Formation for Magnetic Compression/Magnetized Target Fusion," Phys. Rev. Lett. 75, 1953 (1995).

[Logan93] B. Grant Logan, "Inetial fusion reactors using Compact Fusion Advanced Rankine (CFARII) MHD Conversion," Fusion Engineering and Design 22, 151 (1993).

[Miles66] J. W. Miles, "Taylor Instability of a Flat Plate," General Dynamics report GAMD-7335 (1966).

[Milroy82] R.D. Milroy and J.U. Brackbill, "Numerical studies of a field reversed theta pinch plasma", Phys. Fluids 25, 775 (1982). 
[Moir94] R. W. Moir, "Improvements to the HYLIFE-II inertial fusion power plant design, "Fusion Technology 26, 1169(1994).

[Moir95] R. W. Moir, "PACER Revisited: History and Future Challenges," Lawrence Livermore National Laboratory unpublished presentation, Fusion Skunk Works, January 18, 1995.

[Momota92] H.Momota, A. Ishida, Y. Kohzaki et al., "Conceptual design of the D-3He reactor ARTEMIS," Fusion Technology 21, 2307 (1992).

[Monsler92] M. J. Monslex, W. R. Meier, R. L. Bieri, et al., "Results of inertial fusion reactor design studies: Osiris and Sombrero," 14 $4^{\text {th }}$ Intnl. Conf. On Plasma Physics and Cont. Nuc. Fusion (IAEA, 1992).

[Moses79] R. W. Moses, R. A. Krakowski, and R. L. Millex, "A Conceptual Design of the Fast-Liner Reactor (FLR) for Fusion Power," Los Alamos Scientific Laboratory informal report LA-7686-MS (1979).

[Parker93] J. Parker, "A Primer on Liner Implosions with Particular Application to the Pegasus II Capacitor Bank," Los Alamos National Laboratory ATHENA Technical Report No. 1 (November 1993).

[Reinovsky96] R. E. Reinovsky and C. A. Ekdahl, "Development of Imploding Liners With Kinetic Energies of $100 \mathrm{MJ}$ and Their Applications," Proc. VII Intl. Conf. on Megagauss Magnetic Fields and Their Applications, Sarov, Russia, to be published by VNIIEF (August 1996).

[Rej84] D.J. Rej and M. Tuszewski, "A zero-dimensional transport model for field-reversed configurations", Phys. Fluids 27, 1514 (1984).

[Rej86] D. J. Rej, R. D. Milroy, M. Tuszewskj, R. E. Siemon, E. G. Sherwood, K. F. McKenna, R. K. Linford, P. L. Klingner, R. E. Chrien, and W. T. Armstrong, R. E.Chrien, "Experimental studies of fieldreversed configuration translation," Phys. Fluids 29 (3), 852-862 (1986).

[Rej92] D. J. Rej, B. L. Wright, W. J. Waganaar, M. Tuszewski, R. J. Gribble, R. E. Chrien, M. H. Baron, and D. P. Taggart, "High-power magnetic-compression heating of field-reversed configurations," Phys. Fluids B 4 (7), 1909-1919 (1992).

[Robinson89] A. C. Robinson and J. W. Swegle, "Acceleration Instability in Elastic-Plastic Solids (Parts 1 and 2)," J. Appl. Phys. 66 (7), 2838 (October 1989).

[Sheehey96] P. Sheehey, J. Guzik, R. Kirkpatrick. I. Lindemuth, D. Scudder, J. Shlachter, and F. Wysocki, Fusion Technology 30, 1355 (1996).

[Sheehey97] P. Sheehey, J. Hammel, I. Lindemuth, R. Lovberg, R. Riley Jr., D. Scudder, and J. Shlachter, Phys. Fluids B 4, 3698 (1992); P. Sheehey Ph.D. thesis, Los Alamos National Laboratory Report LA12724-T, 1994; P .Sheehey and I. Lindemuth, Phys. Plasmas 4, 146 (1997).

[Siemon80] R. E. Siemon and R. R. Bartsch, "Scaling Laws for FRC Formation and Prediction of FRX-C Parameters," Proc. 3rd Symp. Physics and Technology of Compact Toroids, Los Alamos, New Mexico, December 2-4, 1980, LA-8700-C, p. 172, Los Alamos National Laboratory (1980).

[Siemon86] R. E. Siemon, et. al., "Review of the Los Alamos FRX-C Experiment," Fusion Technology Y(1), 13-37 (1986). 
[Siemon97] R. E. Siemon, "Magnetized Target Fusion - A High-Density Pulsed-Power Approach to Fusion," Los Alamos National Laboratory talk LA-UR-97-764, presented at the Innovative Confinement Concepts Workshop, Marina del Rey, California, March 3-6, 1997.

[Slough92] J. T. Slough, A. Shiokawa, Y. Ito, G. A. Wurden, R. Maqueda, M. Cecik, E. A. Crawford, R. D. Milroy, and A. L. Hoffman, "Confinement and stability of plasmas in a field-reversed configuration," Phys. Rev. Lett. 69 (15), 2212-2215 (1992).

[Slough93] J. T. Slough and A. L. Hoffman, "Stability of field-reversed configurations in the large $s$ experiment (LSX)," Phys. Fluids B 5 (12), 4366-4377 (1993).

[Slough95] J. T. Slough, L. C. Steinhauer, R. Maqueda, R. D. Milroy, and A. L. Hoffman, "Transport, energy balance, and stability of a large field-reversed configuration, Phys. Plasmas 2 (6) 2286-2291 (1995).

[Spencer83] R. L. Spencer, R. K. Linford, and M. Tuszewski, "Adiabatic compression of elongated fieldreversed configurations," Phys. Fluids 26 (6), 1564-1568 (1983).

[Steinhauer83] L. C. Steinhauer, "Plasma heating in field-reversed theta pinches," Phys. Fluids 26 (1), 254-263 (1983).

[Steinhauer85] L.C. Steinhauer, "Magnetic flux trapping during field reversal in the formation of a fieldreversed configuration", Phys. Fluids 28, 3333 (1985).

[Steinhauer92] L. C. Steinhauer, "Electron thermal confinement in the edge plasma of a field-reversed configuration," Phys. Fluids B 4 (12), $4012-4018$ (1992).

[Steinhauer97] L. C. Steinhauer and A. Ishida, "Relaxation of a two-species magnetofluid," Phys. Rev. Lett. 79 (18), 3423-3426 (1997).

[Sweeney81] M. A. Sweeney, A. V. Farnsworth Jr., " High-Gain, Low-Intensity ICF targets for a charged-particle beam fusion driver," Nuc. Fusion 21, 41 (1981).

[Tuszewski88] M. Tuszewski, "Field Reversed Configurations," Nuclear Fusion 28(11), 2033-2092 (1988).

[Tuszewski88b] M. Tuszewsk, G. A. Barnes, R. E. Chrien, et al., "A semiempirical formation model for field-reversed configurations," Phys. Fluids 31 (12), 3754-3759 (1988).

[Tuszewski91a] M. Tuszewski, D. P. Taggart, R. E. Chrien, D. J. Rej, R. E. Siemon, and B. L. Wright, "I. Axial Dynamics in Field-Reversed Theta Pinches. II: Stability," Phys. Fluids B 3(10), 2856-2870 (October 1991).

[Tuszewski91] M.Tuszewski, D.C. Barnes, R.E. Chrien et al., "Observation of tilt instabilities in fieldreversed configurations," Phys. Rev. Lett. 66, 711 (1991).

[Vekshtein90]G. E. Vekshtein, Rev. Plasma Physics 15, Consultants Bureau, NY (1990).

[Yamada98] M. Yamada, H. Ji, S. Jardin al., "Proposed program SPIRIT," Proc. ICC Workshop, Princeton, 1998. Proc. ICC Workshop, Princeton, 1998. 


\title{
APPENDIX A
}

\section{Why Magnetized Target Fusion Offers A Low-Cost Development Path for Fusion Energy}

Richard E. Siemon

Irvin R. Lindemuth

Kurt F. Schoenberg

\author{
Los Alamos National Laboratory \\ Los Alamos, New Mexico
}

Accepted by Comments on Plasma Physics and Controlled Fusion, December, 1997 


\section{Introduction}

Reasonably priced energy supplies have become an expectation of the developed world and a necessary ingredient for development of Third World countries. The problem of providing large supplies of low-cost energy is a long-term, complex one that requires sustained R\&D efforts, in spite of the shadow cast on long-term R\&D by the federal deficit problem. The role of fusion energy as a power source was thoroughly reviewed and strongly endorsed in 1995 by the President's Committee of Advisors on Science and Technology Fusion Review Panel chaired by John Holdren. He argued [Holdren 95]:

The options available for meeting the world's demand for energy in 2050 and beyond are those already in use -fossil fuels, biomass energy, nuclear fission, hydropower, geothermal energy, wind energy, and solar energy - plus, potentially, nuclear fusion.

In these circumstances, it should be obvious that there is great merit in the pursuit of diversity in energy options for the next century. There are not so many possibilities altogether. The greater the number of these that can be brought to the point of commercialization, the greater will be the chance that overall energy needs can be met without encountering excessive costs from or unmanageable burdens upon any one source.

In the past decade the critical issue for fusion has shifted from one of scientific feasibility to one of commercial viability. The specific problem is that all fusion technologies currently being pursued involve extremely costly facilities for the required steps of further development. In the present international fiscal environment, it is imperative to find a more cost effective development path for fusion energy.

The conventional regime of Magnetic Fusion Energy (MFE), with plasma density $\mathrm{n} \sim 10^{14}$ $\mathrm{cm}^{-3}$ and magnetic field provided by superconducting magnets, has been relatively well explored [Sheffield 96]. Tokamaks are the major devices studied in MFE, and tokamak research has tremendously advanced our understanding of plasma physics. The International Tokamak Experimental Reactor (ITER) design illustrates the technology and cost for an ignited plasma demonstration in the MFE regime. The estimated \$10-billion price for ITER calls into question whether fusion can ever be developed based on tokamaklike technology. Factors of a few, or maybe ten at most, in any parameter such as size, neutron wall loading, and so forth are about all that one can credibly seek in optimizing a tokamak system. Certainly research seeking to reduce the ITER-like system size by factors of a few is extremely important and needs to be pursued. But we strongly suspect that the necessary breakthrough, which would allow fusion to be developed in a more timely and affordable manner, will involve a qualitatively different and significant departure from the MFE tokamak regime and technology.

Another approach to fusion, Inertial Confinement Fusion (ICF), represents a good alternative to MFE in that the regime of density and pressure is completely different, the physics issues are quite distinct, and the technology required has fairly little in common with a tokamak-like system [Lind1 95]. Thus, the issues that are likely to emerge as 
limitations for one approach are unlikely to apply to the other. Unfortunately, the cost of developing ICF is also high. The price of the National Ignition Facility (NIF), which will demonstrate ICF ignition, is over $\$ 1$ billion. The anticipated cost of developing efficient inertial fusion drivers such as heavy ion beams is also high [Bangerter 97]. For the development of fusion energy, something less expensive would obviously be desirable.

\section{A Lower Cost Alternative-Magnetized Target Fusion}

To find a lower cost approach, we start by noting that the cost of development is directly linked to the system size, which in the case of MFE is mostly dictated by the maximum magnetic field strength obtainable with superconducting magnets. The critical constraint with ICF is the costly high-power drivers needed to achieve the extreme conditions of density and pressure.

We also note that countless examples can be found in the magnetic fusion literature showing that fusion reactions can be created in smaller-sized systems if one admits larger magnetic field, higher plasma density, and pulsed operation as with imploding liners [Sherwood 81, Lindemuth 83, Robson 76, Vekshtein 90, Ryutov 96, Gross 76]. In this paper we will review the basic reason for that tendency, and examine some of the consequences. We will conclude that the most interesting regime of density is $\mathrm{n} \sim 10^{20} \mathrm{~cm}^{-3}$, which is high compared with MFE, but low compared with ICF. This density regime at 10 $\mathrm{keV}$ temperature corresponds to megabars of pressure (millions of atmospheres), which is intrinsically pulsed in nature.

We define the intermediate density regime to be Magnetized Target Fusion (MTF). The name is chosen based on two general characteristics that we assume for MTF: 1) as with ICF, PdV work heats the fuel by compressing it inside an imploding wall, or "pusher" in the parlance of ICF, and 2) magnetic field is embedded in the fuel to insulate it from the pusher.

Although numerous variations in approach can be envisioned, we have in mind the magnetically-driven imploding liner method for MTF. In the liner approach:

- fuel with an embedded magnetic field would be preheated and positioned inside a volume of centimeter dimensions, which is surrounded by a thin metal shell (or liner) that will act as the pusher,

- a current introduced on the outer surface of the liner would cause it to implode by selfpinching magnetic forces at a velocity of approximately $10^{6} \mathrm{~cm} / \mathrm{sec}$,

- the liner would be made thick enough that the pinching current does not vaporize it, and therefore the liner would be a flux-conserving metal shell during the implosion,

- at peak compression a significant fraction of the liner kinetic energy would be converted to thermal energy of the fuel, and

- the dwell time of the liner at peak compression and the final fuel density and temperature would be designed to give significant fusion energy generation.

The liner velocity required is termed hypervelocity because the kinetic energy density exceeds the heat of vaporization for liner materials. The technology for precision 
implosions creating millions of atmospheres of pressure is a challenge in its own right. In the 1970s when a number of MTF-related efforts were underway, most of the effort was directed towards developing this demanding technology, and very few integrated tests with a preheated plasma were ever done. In what must be viewed as a serendipitous coincidence, the Department of Energy's Office of Defense Programs (DP) in the last decade has significantly advanced the technology of imploding liners with the same parameters of implosion velocity and kinetic energy as those needed for investigating fusion reactions in the MTF regime. The purpose of the Defense Program work is to study and understand hydrodynamics in the megabar pressure regime and has no connection with nuclear fusion. However, the existence of DP expertise and facilities offers an important near term advantage for resuming MTF research.

The magnetic field to insulate fuel from its surroundings is the essential ingredient of MTF. In fact, the benefit of a magnetic field in a fusion target was recognized in the 40's by Fermi at Los Alamos and at approximately the same time by Sakharov in the former Soviet Union. We will derive below the advantages in terms of reduced energy and power that must be delivered to the fusion fuel. The advantages of MTF can also be expressed in terms of requirements on driver technology. By preheating MTF fuel to between 100 and $500 \mathrm{eV}$, the volume compression needed to reach $10 \mathrm{keV}$ temperature is $100-1000$. The volume compression ratios for ICF are typically 30,000 to 60,000 , which requires a much more precise implosion system. The characteristic implosion velocity for MTF is 0.3-3.0 $\mathrm{cm}$ per microsecond, which is 10 to 100 times smaller than for ICF. The peak pressure for MTF is 1-10 megabars, and for ICF, 100s of gigabars. These impressive differences justify careful examination of ways to introduce a magnetic field.

\section{The Technical Case for Magnetized Target Fusion}

\section{A. Lawson Condition for Pulse Duration and Energy Confinement Time}

In a pulsed system, as opposed to steady-state, the pulse duration, $\tau_{\text {burn, }}$ is an important new variable. The pulse duration determines the amount of fuel that reacts or "burns," given the reaction cross section, leading to an $n \tau_{\text {burn }}$ requirement in a similar way that $n \tau_{\mathrm{E}}$ is determined from power balance in a steady-state system. For deuterium (DT) fuel the thermonuclear reaction rate per unit volume is

$$
\mathrm{R}=\mathrm{n}_{\mathrm{D}} \mathrm{n}_{\mathrm{T}}\left\langle\sigma_{\mathrm{DT}} \mathrm{v}\right\rangle=1 / 4 \mathrm{n}^{2}\left\langle\sigma_{\mathrm{DT}} \mathrm{v}\right\rangle
$$

where $\mathrm{n}_{\mathrm{D}}=\mathrm{n}_{\mathrm{T}}$ is the deuterium and tritium density, $\mathrm{n}=\mathrm{n}_{\mathrm{D}}+\mathrm{n}_{\mathrm{T}}$ is the total ion or electron density, and $\left\langle\sigma_{\mathrm{DT}} v\right\rangle$ is the averaged product of cross section and relative velocity for a Maxwellian velocity distribution. At $10 \mathrm{keV},\left\langle\sigma_{\mathrm{DT}} \mathrm{v}\right\rangle \cong 10^{-16} \mathrm{~cm}^{3} / \mathrm{sec}$. The total density decreases at the rate $2 \mathrm{R}$ as the fuel is consumed, and the frequency of fusion reactions per ion for either deuterium or tritium ions is given by $2 R / n$ :

$$
(\mathrm{dn} D / d t) / n_{D}=(d n / d t) / n=1 / 2 n\left\langle\sigma_{D T} v\right.
$$


Assuming for simplicity that DT fuel is held at constant temperature so that $\left\langle\sigma_{\mathrm{DT}} v\right\rangle$ is constant in time while it burns, Eqn. 2 can be integrated to give

$$
\mathrm{n} / \mathrm{n}_{0}=1 /\left(1+\mathrm{n}_{0} \tau_{\text {burn }}<\sigma_{\mathrm{DT}} \mathrm{v}>/ 2\right)
$$

where $\tau_{\text {burn }}$ is the burn time. Equation 3 can be recast in terms of $f$, the fractional burnup of fuel, as:

$$
\mathrm{f} /(1-\mathrm{f})=\mathrm{n}_{0} \tau_{\text {burn }}<\sigma_{\mathrm{DT}} \mathrm{v}>/ 2
$$

where $f \equiv 1-n / n_{0}$. For complete burnup, the gain would be $G_{\max }=300$ at $10 \mathrm{keV}$. This is simply the ratio of energy for a $14.1 \mathrm{MeV}$ neutron and $3.5 \mathrm{MeV}$ alpha divided by the $60 \mathrm{keV}$ of thermal energy for a DT ion pair with electrons.

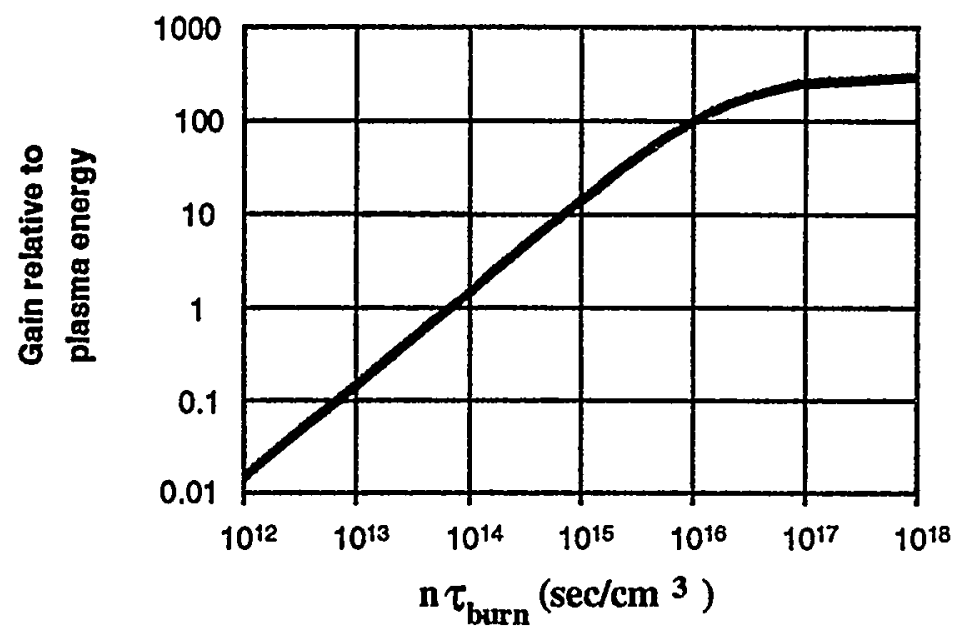

Figure 1. Fusion energy output relative to plasma energy vs. the product of density and burn time.

As a function of burn time, the gain plotted in Fig. 1 is $\mathrm{G}_{\max }$ times the fractional burnup. We can define a Lawson condition using Fig. 1 . With $\mathrm{n} \tau_{\text {burn }} \sim 3 \times 10^{14} \mathrm{~cm}^{-3} \mathrm{sec}$ the gain relative to thermal energy is around five, enough to allow for net gain with realistic efficiencies. The net gain relative to initially stored electrical energy is the gain of Fig. 1 times the efficiency of heating fuel to $10 \mathrm{keV}$ temperature. For example, if $50 \%$ of the stored electrical energy is converted to liner kinetic energy [Gerwin 78], and 50\% of the liner kinetic energy is converted to thermal plasma energy at peak compression, then the net gain would be $1 / 4$ of the gain plotted in Fig. 1 .

A plasma heated to $10 \mathrm{keV}$ will cool by numerous mechanisms. The total power losses per unit volume are conventionally written as $3 \mathrm{nT} / \tau_{\mathrm{E}}$, where $\tau_{\mathrm{E}}$ is the global energy confinement time. In deriving Fig. 1 we ignored losses, which is equivalent to assuming $\tau_{\mathrm{B}} \gg \tau_{\text {burn }}$. To obtain the minimum possible system size for the purpose of low-cost development, we would require $\tau_{\mathrm{E}} \sim \tau_{\text {burn }}$. That is, if $\tau_{\mathrm{E}}$ were much less than $\tau_{\mathrm{burn}}$ the fuel would cool before it burned. On the other hand if $\tau_{E}$ were much larger than $\tau_{\text {burn }}$, the 
plasma should be made smaller to equalize the two, which requires less energy, assuming the energy confinement time increases with system size. For approximate estimates, the relevant energy confinement time and the burn time should both satisfy a Lawson-like $n \tau$, which we will take for the purposes of demonstrating feasibility to be the same as ITER, and approximately an energy breakeven condition according to Figure 1:

Lawson requirement: $\mathrm{n} \tau \sim \mathrm{n} \tau_{\mathrm{E}} \sim \mathrm{n} \tau_{\text {burn }} \sim 3 \times 10^{14} \mathrm{~cm}^{-3} \mathrm{sec}$

This $\mathrm{n} \tau_{\mathrm{E}}$ corresponds to $1.5 \%$ burnup fraction in a pulsed system.

\section{B. Pressure of High-Density Fuel Dictates Pulsed Technology}

The first requirement for containing fuel is equilibrium or pressure balance to prevent the fuel from expanding during the required burn time. There are a continuum of possibilities ranging from ICF with zero magnetic field where pressure is supported by the inertia of surrounding low-temperature fuel, to full magnetic confinement where plasma pressure is less than or equal to the confining magnetic pressure. In the MTF regime we consider the possibility where plasma pressure is larger than or equal to the magnetic field pressure, because the main role of magnetic field is insulation and not confinement.

Broadly speaking, the relevant technology changes as the density increases. We assume $\mathrm{Ti}$ $\sim \mathrm{Te} \sim 10 \mathrm{keV}$. At densities from $10^{14} \mathrm{~cm}^{-3}$ up to about $10^{16} \mathrm{~cm}^{-3}$ plasma pressure can be contained by superconducting magnets, where the higher density corresponds to magnetic confinement with $\beta=1$. Plasma $\beta \equiv 2 \mathrm{nkT} /\left(\mathrm{B}^{2} / 8 \pi\right)$, where $B$ is the magnetic field. At pressure or density too high for superconductors, pulsed magnets can be used up to pressures that fracture known materials. Strength limitations set an upper limit on the density at about $10^{18} \mathrm{~cm}^{-3}$. This density corresponds to magnetic field of about $1 \mathrm{MG}$ if magnetic pressure confines the plasma. To date, the largest magnetic fields reported are pulsed fields of about $20 \mathrm{MG}$, which can be obtained by imploding liners [Pavlovskii 96]. If $20 \mathrm{MG}$ were used for plasma confinement, the corresponding maximum density is around $10^{21} \mathrm{~cm}^{-3}$. Above that density, plasma pressure must be held by the inertia of material walls, although magnetic field can be utilized for its insulating properties. For ICF the density of the ignited hot spot is expected to be about $10^{25} \mathrm{~cm}^{-3}$, which corresponds to a pressure of $200 \mathrm{Gbar}$. We see that the technology for fusion changes radically as one moves from MFE density to ICF density.

\section{Fusion Fuel Diffuses Before Burning}

Another basic point useful to recall for the following discussion is that $\sigma_{\mathrm{DT}}$, the cross section for fusion, is much smaller than $\sigma_{\mathrm{C}}$, the cross section for Coulomb scattering, almost independent of density. By definition the frequency of collisions is given by the product of cross section and flux. The rate of fusion reactions is given by the right-hand side of Eqn. 3:

$$
\text { Frequency of fusion reactions }=1 / 2 n<\sigma_{D T} v>
$$


The effective fusion cross section can be taken as $\left\langle\sigma_{D T} v>v_{i}, \sim 1\right.$ barn $\left(10^{-24} \mathrm{~cm}^{2}\right)$ at $10 \mathrm{keV}$ where $v_{i}$ is the ion thermal speed. Similarly the Coulomb collision frequency can be written as a product of the Coulomb cross section and particle flux, $\mathrm{n}$ multiplied by $v_{\mathrm{i}}$ :

$$
\text { Ion-ion Coulomb collision frequency }=v_{\mathrm{ii}}=1 / \tau_{\mathrm{ii}}=\mathrm{n} v_{\mathrm{i}} \sigma_{\mathrm{C}}
$$

Thus at $10 \mathrm{keV}$ and $10^{14} \mathrm{~cm}^{-3} \sigma_{\mathrm{C}} \sim 7000$ barns. This Coulomb collision frequency, or reciprocal of the ion-ion collision time, is extensively discussed in the standard textbooks. Because of the accumulated effects of small-angle scattering, the frequency of Coulomb collisions is proportional to $\ln \Lambda$, a factor that depends weakly upon temperature and density. The Coulomb logarithm is often taken as a constant about equal to 20 , but even for rough estimates we will calculate $\ln \Lambda$ when it arises, because the range of density we will consider $\left(10^{14}-10^{26} \mathrm{~cm}^{-3}\right)$ corresponds to $\ln \Lambda$ changing by more than a factor of 3 .

At a temperature of $10 \mathrm{keV}$, the cross section or frequency for Coulomb scattering is larger than the cross section or frequency of fusion reactions by a factor of 2000-6000 for density between $10^{26} \mathrm{~cm}^{-3}$ and $10^{14} \mathrm{~cm}^{-3}$ respectively. Therefore, the number of collisions $(\mathrm{N})$ that occur during a burn time is calculated to be:

$$
\mathrm{N}=\tau_{\text {burs }} / \tau_{\mathrm{ii}}=2 \mathrm{f} \mathrm{v}_{\mathrm{i}} \sigma_{\mathrm{C}} /\left\langle\sigma_{\mathrm{DT}} \mathrm{v}\right\rangle
$$

For $\mathrm{n} \tau_{\mathrm{E}}=3 \times 10^{14} \mathrm{~cm}^{-3} \mathrm{sec}$, the burn time is between 60 and 180 ion-ion collision times as density varies from $10^{26} \mathrm{~cm}^{-3}$ to $10^{14} \mathrm{~cm}^{-3}$.

In summary, we conclude that, independent of the fuel density over a wide range of density, collisional diffusive processes are unavoidable when fusion fuel is assembled for a time long enough to produce energy gain.

\section{The Nature of Energy Diffusion}

Even if fuel is held in pressure balance for the necessary burn time, it has been historically difficult to achieve the required global energy confinement time. Much of MFE fusion research has been devoted to understanding the many modes of plasma motion that transport energy in addition to classical collisional processes. With ICF, there is less uncertainty about loss processes, because the absence of a magnetic field simplifies the transport physics. In that case electron thermal conduction is the dominant loss process. In the ICF approach parameters are chosen so that even electron thermal conduction is consistent with the Lawson condition. One could say that ICF is the "worst case" for thermal losses when compared with any type of magnetic configuration.

Classical diffusion. We review now the lower bound on energy confinement represented by classical diffusion. In MFE fusion literature, the global energy confinement time is usually expressed in terms of thermal diffusivity:

$$
\tau_{E} \sim a^{2} / x
$$


where $a$ is the characteristic dimension across which heat diffuses and $\chi$ is the thermal diffusivity. The value of $\chi$ (same as thermal conductivity divided by density) is derived by calculating the energy flux in the presence of a temperature gradient.

Thermal diffusion can also be viewed as a random walk of particles. After each collision, a particle moves one step at random either up or down the temperature gradient. Heat conduction is the diffusion of cold particles up the gradient and hot particles down the gradient with no net flux of particles. The essential feature of the random walk is that after $\mathrm{N}$ collisions, there is a binomial distribution for particle location, and it has a width proportional to $\mathrm{N}^{1 / 2}$. If the step size is $\lambda$, then the standard deviation of the distribution of particle locations after $\mathbf{N}$ collisions is $a$ given by

$$
a=\mathrm{N}^{1 / 2} \lambda \text {. }
$$

If the collision time is $\tau$, the number of collisions is $N=t / \tau$, so we can also write Eqn. 9 as

$$
t=(a \lambda)^{2} \tau
$$

Eqn. 9 indicates that if $\mathrm{N}$ collisions are needed before heat dissipates, then the fuel must have a characteristic size greater than $a$. Equivalently, Eqn. 10 gives the time to dissipate heat (energy confinement time) in terms of the number of steps across the characteristic size, $(a / \lambda)$, and the time per step or collision time.

Classical diffusion without a magnetic field. To apply the random-walk argument to electron thermal conduction, we equate the step size to a plasma mean free path $\lambda$. Electrons have a larger thermal speed and a shorter collision time, such that the mean free path $\lambda$ is the same for either ions or electrons:

$$
\lambda=1 / n \sigma_{\mathrm{C}}=v_{\mathrm{i}} \tau_{\mathrm{ii}}=\mathrm{v}_{\mathrm{e}} \tau_{\mathrm{ee}}
$$

where $v_{i, e}$ is the ion, or electron, thermal speed. Electrons collide more frequently by a factor of $\left(\mathrm{m}_{\mathrm{i}} / \mathrm{m}_{\mathrm{e}}\right)^{12}$, or about 60 for a DT mixture. Therefore, if we consider high density where ions make about 60 collisions, then electrons make about 3600 collisions during the fusion burn time. The size of a plasma with burn time long enough to allow 3600 electronelectron collisions is

$$
a=(3600)^{1 / 2} \lambda .
$$

For ICF, where the ignition hot spot density is about $10^{25} \mathrm{~cm}^{-3}$, the mean free path is 0.7 microns; this simple estimate of Eqn. 12 for hot spot radius is 42 microns. More detailed calculations [Lindl 95] give about the same value.

Classical diffusion with a magnetic field. To apply the random-walk argument to magnetized plasma is more difficult, because the step size depends upon complicated particle orbits in the magnetic field. However, for poloidal-field dominated configurations 
like the Reversed Field Pinch, the spheromak, and the Field-Reversed Configuration (FRC), and for tokamaks, detailed studies give the simple prescription that the step size can be taken as the ion gyro radius calculated in the poloidal magnetic field [Boozer 83]. (In a torus the toroidal direction is the long way around the torus, and the poloidal direction is the short way around.) In the direction perpendicular to a magnetic field, the classical ion heat conduction dominates because the ions have a larger gyro radius. Therefore, we can estimate that the minimum required size of a fusion system to diffuse heat slowly enough to meet Lawson, say 180 ion-ion collision times, is

$$
a=(180)^{1 / 2} \mathrm{r}_{\mathrm{i}},
$$

where $r_{i}$ is the ion gyro radius in the poloidal magnetic field. The tokamak banana-regime formulas for neoclassical transport theory give about $20 r_{i}$ instead of the approximate estimate of $13 r_{i}$ given by Eqn. 13. Because of anomalous transport, the design radius of ITER is about 5 times larger than the neoclassical limit (i.e. $a_{i t a r} \cong 100 r_{i}$ ).

\section{E. Characteristic Step Sizes Decrease as Density Increases}

Comparing Eqns. 8 and 10, we see that $\chi$ has the form of a step size squared times a collision frequency. For classical transport,

$$
\begin{aligned}
& \text { Electron thermal conduction: } x_{0} \sim \lambda^{2} v_{e e} \\
& \text { Ion cross field transport: } x_{i} \sim r_{i}^{2} v_{i i} .
\end{aligned}
$$

The mean free path $(\lambda)$, which depends on temperature and density, is plotted in Fig. 2 for $10 \mathrm{keV}$ temperature. The gyro radius $\left(r_{i}\right)$, which depends mainly on density, is also plotted in Fig. 2 , assuming constant poloidal beta $\left(\beta_{i}\right)$, where $\beta_{i}$ is the ratio of ion pressure to poloidal field pressure $\left(\beta_{\mathrm{i}}=8 \pi \mathrm{nk} \mathrm{T}_{\mathrm{j}} / \mathrm{B}_{\mathrm{p}}{ }^{2}\right)$. The density dependence can be seen by writing the gyro radius as:

$$
r_{i}=v_{i} / \omega_{c i}=\left(c / \omega_{p i}\right) \beta_{i}{ }^{1 / 2}
$$

where $\omega_{c i}$ is the ion cyclotron frequency in the poloidal magnetic field, $c$ is the speed of light, and $\omega_{\mathrm{pi}}$ is the ion plasma frequency,

$$
\omega_{p i}=\left(4 \pi t n e^{2} / m_{i}\right)^{1 / 2} \text { (cgs units). }
$$

Poloidal beta in tokamaks and the above mentioned configurations is observed not to differ much from unity.

In the spirit of a survey of minimum system size for fusion, Fig. 2 gives useful guidance. The dimensions of a system without magnetic insulation become unacceptably large at low density. The classical limit for the size of a magnetized plasma is seen to be quite small as density increases. If the anomaly factor assumed in the ITER design, and observed with tokamaks having density in the vicinity of $10^{14} \mathrm{~cm}^{-3}$, were to apply at higher density, then 
Lawson should be possible at $10^{20} \mathrm{~cm}^{-3}$ in a tokamak with a minor radius of $2.8 \mathrm{~mm}$ ! This dramatic reduction in size at higher density provides much of the motivation for MTF.

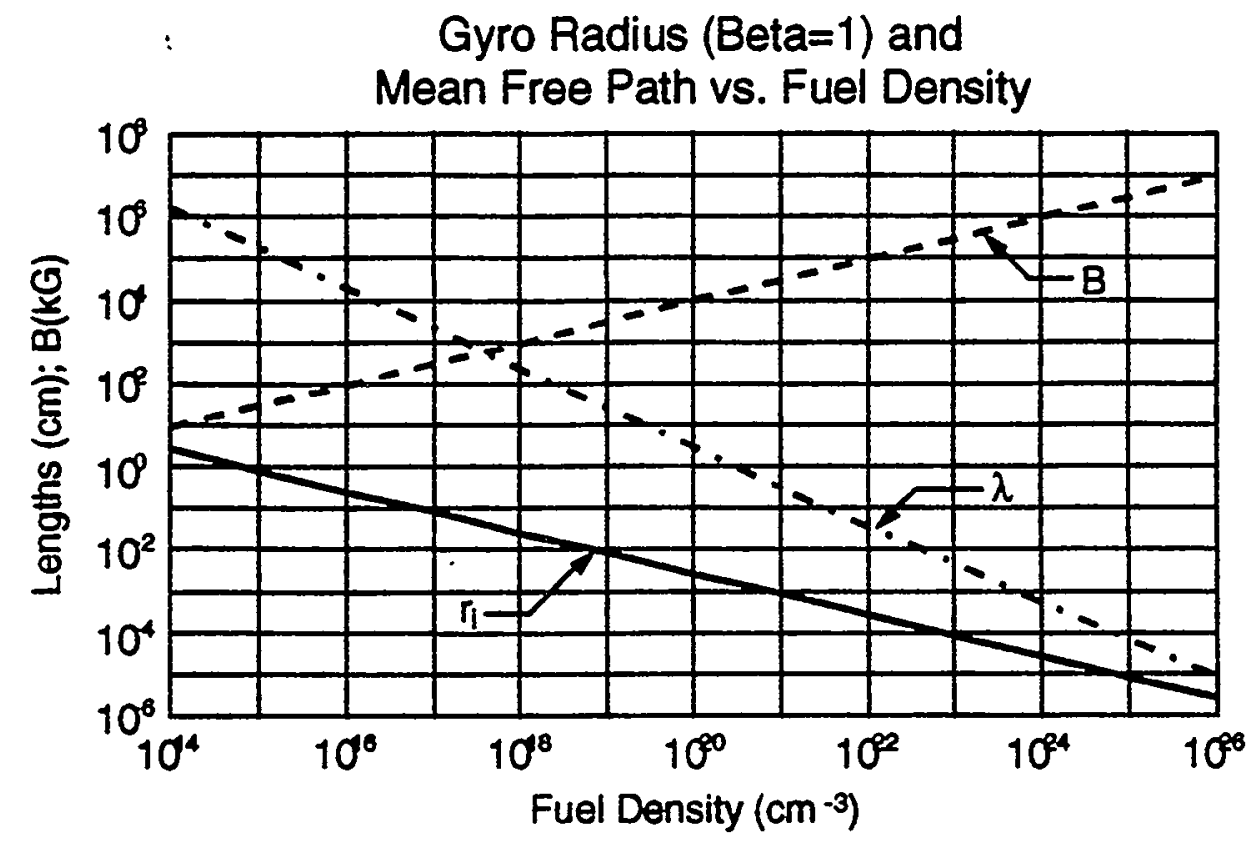

Figure 2. Plots of characteristic step sizes and poloidal magnetic field strength assuming poloidal beta $=1 \mathrm{vs}$. fuel density for a plasma with $10 \mathrm{keV}$ temperature.

Speculation on anomalous transport. Anomalous transport mechanisms are still a subject of unfinished research. Clearly, all possibilities cannot be anticipated, but the following can be noted. Generally the form of $\chi$ is a product of characteristic lengths times a frequency. The characteristic lengths in a plasma normally identified are $\lambda, \lambda_{D}$, $c / \omega_{\mathrm{p}}, c / \omega_{\mathrm{pe}}, \mathrm{r}_{\mathrm{i}}$, and $\mathrm{r}_{\mathrm{e}}$. As already noted, $c / \omega_{\mathrm{pi}}$ and $r_{\mathrm{i}}$ are only different by a factor of order unity, and therefore the gyro radius in Fig. 2 is also approximately the same as $c / \omega_{\mathrm{pi}}$. The gyro radius $r_{e}$ (and thus c/ $/ \omega_{p e}$ ) is smaller than the gyro radius $r_{i}$ by a factor of $\left(m_{i} / m_{e}\right)^{1 / 2}$. The Debeye length $\lambda_{D}$ has the same density dependence as the electron gyro radius. Therefore the variation of all the usual characteristic lengths with density is correctly inferred from Fig. 2, and a reasonable conjecture is that the tendency towards smaller size at higher density is true for anomalous transport as well as for classical transport.

\section{Plasma Energy Reduced at High Density}

To quantify the variation of diffusion step sizes with density in terms that come closer to economic value, we show in Fig. 3 the thermal energy contained by a plasma with characteristic dimension of $a$. Three different configurations are included in Fig. 3: ICFrelevant unmagnetized fuel, tokamaks, and a generic MTF plasma taken to be a compact torus (CT). We assume that when density is varied for a given configuration, size is adjusted to be the minimum necessary to provide $n \tau_{E}=3 \times 10^{14} \mathrm{~cm}^{-3} \mathrm{sec}$ at $10 \mathrm{keV}$ temperature. Specific assumptions for each configuration are summarized in the table following Fig. 3. 


\section{A. ICF Energy Requirements}

For ICF we see a very strong dependence of energy upon density, and thus the importance of compressing to high density. By compressing to density of approximately $10^{25} \mathrm{~cm}^{-3}$, the energy in the hot spot according to Fig. 3 is approximately $30 \mathrm{~kJ}$, which is similar to the value anticipated in the design of NIF [Lind1 95]. Achieving such a high density requires an implosion velocity of about $30-40 \mathrm{~cm}$ per microsecond and a radial convergence of between 30 and 40. The NIF laser design, with $1.8 \mathrm{MJ}$ and $500 \mathrm{TW}$, has enough energy and power to produce these conditions even with the inefficiency of indirect drive. However, if the hot-spot density were to be reduced, the energy requirements would be considerably increased as shown in Fig. 3, and the power requirements would also be increased to achieve the same $n \tau_{\mathrm{E}}$. Thus, the ICF approach utilizes very high density to achieve fusion with minimum energy, but the driver requirements are extremely demanding and expensive.

\section{B. Tokamak Energy Requirements}

Tokamaks are included in Fig. 3 for academic interest, even though high-density operation of a tokamak-like configuration is not being considered. The poloidal magnetic field required at any given density is plotted in Fig. 2 . For the assumed value of safety factor $(q)$ and aspect ratio, the toroidal field required would be approximately a factor of ten higher than the poloidal field. Thus, the magnetic energy would be 100 times as large as the plasma thermal energy plotted in Fig. 2. The cost of a tokamak is well known to be strongly tied to the cost of the magnets.

The important aspect of the tokamak is that much more is known about transport than for any other configuration. A useful summary of tokamak transport formulas can be found in the textbook by Kadomtsev [Kadomtsev 92]. We plot both the classical limit for confinement (neoclassical in the banana, transition, and Pfirsch-Schluter regimes as density increases) and some empirically based models for anomalous transport. The anomalous transport curves show the anticipated tendency that system size becomes small at increasing density. One concludes from these plots that if the technology were available to operate tokamaks at higher density, the size and cost could be reduced.

\section{MTF Energy Requirements.}

For MTF compression by a liner, there are many possible magnetic configurations. To make estimates for Fig. 3, we have chosen a compact toroid (CT) plasma as generic for any magnetic configuration. Specifically the CT curves in Fig. 3 are calculated assuming the plasma is an FRC, which has ideally only poloidal magnetic field [Tuszewski 88]. Similar values apply to a spheromak. In that case a toroidal field comparable in magnitude to the poloidal field of the FRC would be required [Jarboe 94]. CTs require more energy than a tokamak at a given density because CTs need more volume to achieve the same effective radius or insulating distance. A prolate FRC, as is commonly studied in experiments, has an effective radius equal to the distance from the field null to the outer edge, which is 
approximately 0.3 of the small radius of the prolate spheroid. Thus the FRC estimate for energy may be conservatively high in Fig. 3, although modeling of wall-plasma interactions tend to show spatial profiles that resemble an FRC-like profile (Siemon 97).

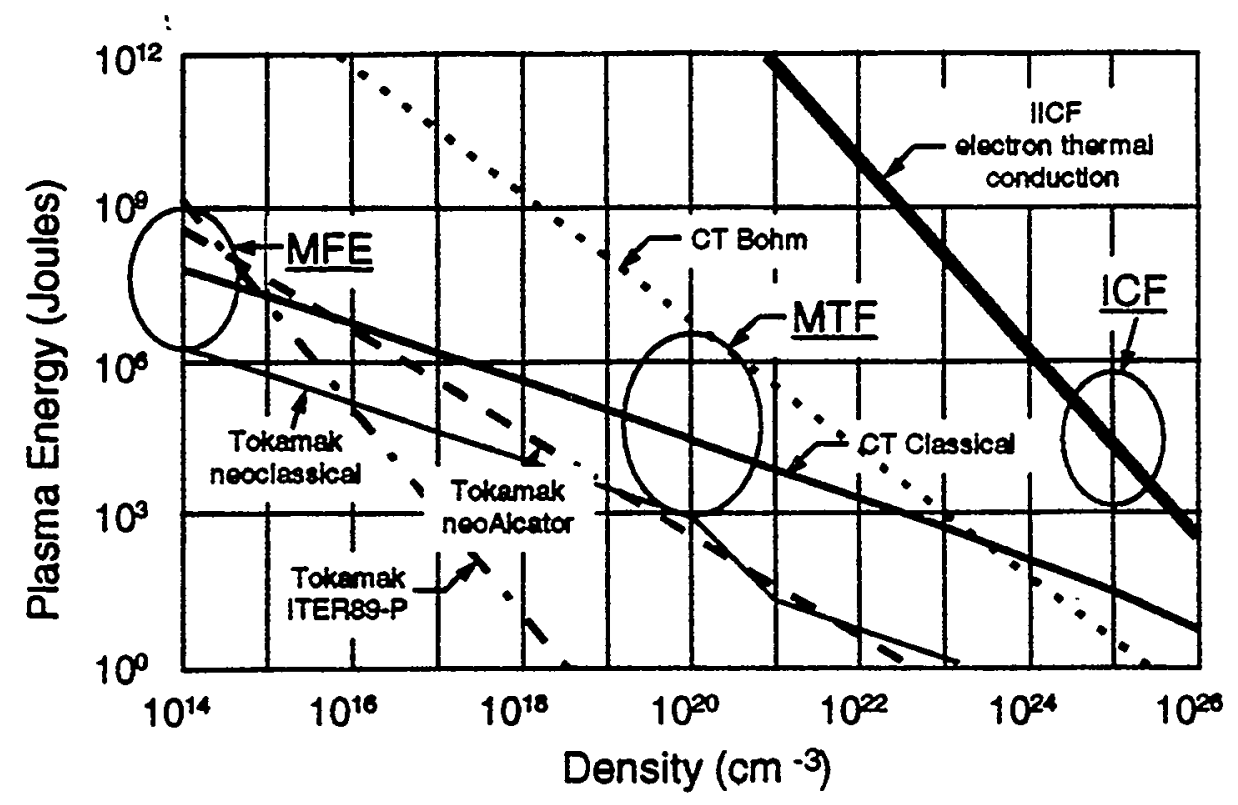

Figure 3. Energy requirements vs. fuel density for various configurations and transport assumptions assuming $n \tau_{E}=3 \times 10^{14} \mathrm{~cm}^{-3} \mathrm{sec}, T=10 \mathrm{keV}$, and poloidal $\beta=1$.

\begin{tabular}{|l|l|l|}
\hline Configuration & Transport & Comments \\
\hline ICF & Electron thermal conduction & $\begin{array}{l}\text { Spherical plasma with size } \\
\text { given by Eqn. 12. Density } \\
\text { of } \sim 10^{25} \mathrm{~cm}^{-3} \text { corresponds } \\
\text { to NIF. }\end{array}$ \\
\hline Tokamak & $\begin{array}{l}\text { Neoclassical, anomalous } \\
\text { neo-Alcator, and anomalous } \\
\text { ITER-89P }\end{array}$ & $\begin{array}{l}\text { Aspect ratio (2.9), poloidal } \\
\text { beta (1.0), and safety factor } \\
\text { q (3.0) are held constant at } \\
\text { ITER-like values. }\end{array}$ \\
\hline Compact Torus (CT) & Classical or Bohm & $\begin{array}{l}\text { Geometry of a prolate FRC } \\
\text { assumed for illustration } \\
\text { with length to diameter ratio } \\
\text { of 3. }\end{array}$ \\
\hline
\end{tabular}

The amount of energy required for fusion conditions depends upon the global energy confinement time. Fig. 3 indicates that compressed plasma energy between about $30 \mathrm{~kJ}$ and $10 \mathrm{MJ}$ is required in the MTF regime (density of $10^{20} \mathrm{~cm}^{-3}$ ), if plasma transport is between classical and Bohm. For the larger Bohm requirement of $10 \mathrm{MJ}$, the required liner kinetic energy would be tens of MJs, a few times the final plasma energy. One striking difference between the MFE and MTF regimes of density is that Bohm is an acceptable possibility at MTF density, while as shown in Fig. 3, Bohm is totally unacceptable at $10^{14} \mathrm{~cm}^{-3}$. 


\section{Comments on Bohm Diffusion}

The curve labeled Bohm deserves additional comment. In the early days of fusion research Bohm was introduced as an'empirical diffusivity [Spitzer 62] equivalent to the following:

$$
\chi_{\text {вонм }}=\chi_{\mathrm{i}}\left(\omega_{\mathrm{ci}} \tau_{\mathrm{ij}}\right) / 16 \text {, }
$$

where $\omega_{\mathrm{ci}} \tau_{\mathrm{ii}}=\lambda \mathrm{r}_{\mathrm{i}}$ is the magnetization parameter. The factor of 16 has no theoretical basis. It is interesting to note that apart from the factor of $16, \chi_{\text {вонм }}$ is the geometric mean or logarithmic average of $\chi_{i}$ and $\chi_{e}$ given in Eqns. 14 and 15. Thus Bohm can be thought of as intermediate between classical magnetized and unmagnetized confinement. Kadomtsev describes how there are situations where macroscopic convection can lead to energy transport with a global Bohm confinement time [Kadomtsev 92]. Studies of wall-confined MTF-type plasma by Vekshtein show how classical confinement can lead to a Bohm-like scaling [Vekshtein 90]. Even more interesting is that experimental data from a number of carefully studied magnetic configurations, including Reversed Field Pinches, spheromaks, and FRCs, is generally as good as Bohm or better.

Global energy confinement time can be worse than Bohm when other non-diffusive processes dominate. Examples are radiation because of impurities, or plasma flow out of the system at a speed comparable to the thermal speed. Radiation by impurities is always a concern and places an upper limit on the allowed impurity concentration. Plasma flow cannot be ruled out in general, but the conjecture here is that target plasma configurations can be found for which a pressure equilibrium exists between the metal liner boundary and the fuel, and thus flow is reduced to nothing worse than convective motions. Close proximity of a conducting boundary should provide a stabilizing influence on magnetohydrodynamic modes, especially since magnetic fields do not penetrate a conducting boundary on the short time scale of interest for MTF. Spheromaks and FRCs are two examples of CTs for which there are data to support this conjecture. We conclude Bohm represents a reasonable, even conservative, expectation for achievable global energy confinement based on previous experimental results, assuming impurities can be avoided by careful experimental technique.

\section{The Size and Cost of Ignition Facilities}

Only a rough connection can be made between cost and plasma energy plotted in Fig. 3 . For each of the configurations, however, one would expect that the indicated reduction of energy as density increases would result in a reduction. of costs for the required facility to create the ignition-grade plasma. Even an approximate connection is adequate for present purposes, given the many decades of system size plotted in Fig. 3. Note that the left-hand scale varies by 12 orders of magnitude. We list in Table 1 costs for recently designed ignition-class facilities in each of the regimes of MFE, ICF, and MTF.

In the case of MTF we base the cost for an ignition facility upon the ATLAS pulsed-power facility, recently designed and under construction by Defense Programs at Los Alamos [Trainor 97]. ATLAS should be able to deliver 5-10 MJ to an imploding liner, which makes it suitable for a considerable range of possible MTF experiments. Although the 
primary mission of ATLAS is not MTF, a reasonable number of additional experiments to test MTF are consistent with current plans for the facility. For the purpose of estimating MTF ignition-grade facility costs, we assume that 1) the 35-MJ of stored energy in ATLAS is enough to implode a liner-plasma configuration to ignition (see Fig. 3), and 2) the additional cost for the plasma target preparation is small compared with the \$50-million cost of the ATLAS facility. The purpose of Table I is to compare facility costs needed for a fusion energy development program. The fact that ATLAS is being built for other reasons is simply a fortunate circumstance. The research effort expended to date on MTF has been minuscule compared with the other two approaches to fusion, and so the cost of achieving ignition conditions is obviously much less certain. However, the advantage appears so large that the accuracy of the estimate is not very important.

Table 1. Approximate Cost of Ignition Facilities

\begin{tabular}{|lll|}
\hline Concept & $\begin{array}{l}\text { Plasma Thermal } \\
\text { Energy }\end{array}$ & Facility Cost \\
\hline MFE/TTER & $1 \mathrm{GJ}$ & $\$ 10$ billion \\
\hline ICF/NIF & $30 \mathrm{~kJ}$ & $\$ 1$ billion \\
\hline MTF/ATLAS & $\sim 10 \mathrm{MJ}$ & $\sim \$ 50$ million \\
\hline
\end{tabular}

\section{Near Term Prospects for MTF Research}

\section{A. Typical MTF Parameters}

The main points of this paper, which are contained in Fig. 3 and Table 1, argue for starting a new thrust in fusion energy research. In this section we discuss some aspects of how to begin that effort. Our concept for a liner-driven plasma implosion suggests approximate values for initial and final plasma parameters as given in Table 2.

Table 2. Representative Conditions for an Adiabatic Implosion

$\begin{array}{llll}\text { Parameter } & \begin{array}{l}\text { Desired Final } \\ \text { Conditions }\end{array} & \begin{array}{l}\text { Required Initial Plasma } \\ \text { if } \mathrm{K}_{\mathrm{v}}=100\end{array} & \begin{array}{l}\text { Required Initial Plasma } \\ \text { if } \mathrm{K}_{\mathrm{v}}=1000\end{array} \\ \begin{array}{l}\text { Temperature } \\ \text { Density }\end{array} & \begin{array}{l}10 \mathrm{keV} \\ 10^{20} \mathrm{~cm}^{-3}\end{array} & \begin{array}{l}460 \mathrm{eV} \\ 10^{18} \mathrm{~cm}^{-3}\end{array} & 100 \mathrm{eV} \\ \text { B Field } & 10 \mathrm{MG} & 100 \mathrm{kG} & 10^{17} \mathrm{~cm}^{-3} \\ \text { Liner inner radius } & 5 \mathrm{~mm} & 5 \mathrm{~cm} & 100 \mathrm{kG} \\ \end{array}$

To illustrate the required initial target-plasma conditions, we assume adiabatic compression ( $\mathrm{pV} \gamma=$ const) with a volumetric compression $\mathrm{K}_{\mathrm{v}}=100$ or 1000 , corresponding to cylindrical, or spherical, radial compression of 10 respectively. The adiabatic approximation is justified according to time-dependent calculations taking thermal and radiation losses into account [Lindemuth 83], and the parameter space for MTF is found to be quite large, assuming an implosion velocity on the order of $10^{6} \mathrm{~cm} / \mathrm{sec}$. 


\section{B. Target Plasma Possibilities.}

Among the many possible magnetic configurations that would be possible for the target plasma, the ones currently receiving attention in our awareness are: 1) the MAGO-type of accelerated diffuse-z-pinch plasma [Lindemuth, 96], 2) an expanded high-density-fiber z pinch inside a conducting boundary [Wysocki 97], and 3) compact toroids [Ryutov 96]. An approach that uses energy from a high-power e-beam driver to form a magnetized plasma has also been reported [Chang 78].

Extensive research on compact toroids, the spheromak and Field-Reversed Configuration, began in about 1980. The review articles by Tuszewski and Jarboe have hundreds of references [Tuszewski 88, and Jarboe 94]. By definition, a CT is a self-contained magnetized plasma that can be moved from one spatial location to another. Thus, CTs are an obvious candidate for inserting a plasma target into an imploding metal liner.

Unfortunately, most fusion-related liner research ended about the same time that CT work began, so most of the information gained from CT research was not available to the early liner researchers. A few experiments studying the implosion of an FRC-type of CT were done in Russia [Kurtmullaev 82]. Most CT research was done at much lower density than is needed for MTF. The RACE experiments at LLNL are a notable exception [Hammer 91]. There is no obvious problem in forming CTs at higher density, and experiments to move in that direction would be desirable.

The MAGO and expanded fiber $\mathrm{z}$ pinch are diffuse z-pinch magnetic configurations. The outstanding attraction of these approaches is that the technology for plasma formation is reasonably compatible with liner implosion technology, and is less complicated than for CTs. For MAGO at least, plasma density and temperature appear suitable for proceeding with MTF implosion experiments [Lindemuth 95]. More refined measurements are still needed to characterize global energy confinement in both the MAGO and expanded fiber plasmas. The diffuse $\mathrm{z}$ pinch has well known limitations with regard to stability, and containment of energetic particle orbits. However, simulations show [Sheehey 89] that an unstable plasma inside a conducting boundary can evolve to a stable state (known as a Kadomtsev-stable profile). In such a state, the energy confinement may be adequate on the time scale of an MTF implosion. The fact that most alpha particles generated near peak compression would be lost is not a major consideration for the batch-burn approach we have assumed for MTF.

\section{Liner Technology and Facilities are Available.}

The advances in liner technology of the past few years are impressive [Chernyshev 97]. More than enough liner velocity and implosion symmetry has been demonstrated compared with the detailed requirements for an MTF liner system discussed elsewhere [Lindemuth 96, Siemon 97, Ryutov 96, and Schoenberg 98]. A quasi-spherical implosion of unmagnetized plasma has also been reported [Degnan 96].

A number of existing facilities supported by DOE's Defense Programs and DOD would be suitable for a variety of MTF experiments. These include the $Z$ capacitor bank at Sandia National Laboratory, the Shiva Star capacitor bank at Phillips Air Force Laboratory, the 
Pegasus capacitor bank at Los Alamos National Laboratory, the Ranchero explosivelydriven electrical generators at Los Alamos, and the ATLAS capacitor bank under construction at Los Alamos.

These facilities and expertise allow significant leveraging of research dollars, which gives additional incentive for MTF research.

\section{Major Technical Issues.}

MTF can be conceptually separated into three inter-related aspects: target plasma formation and confinement properties, liner-driver implosion, and target-plasma compression. The major technical issues are:

Issues of Target Plasma Formation and Confinement Properties

- plasma parameters on the proper adiabat for heating to ignition

- suitable magnetic topology for magnetohydrodynamic stability and adequate thermal insulation

- plasma-wall interactions leading to high- $Z$ impurities and concomitant plasma radiation losses

Liner-Driver Implosion Issues

- symmetric implosions of a liner at approximately $10^{6} \mathrm{~cm} / \mathrm{s}$ (a velocity well within the range of what has been demonstrated in Defense Program experiments).

- development of liner implosion configurations that match target-plasma requirements for a conducting boundary throughout the implosion

- convergence ratios of roughly 10 in a stable quasi-spherical geometry

Target Plasma Compression Issues

- technical compatibility between plasma formation and liner-implosion technologies

- accelerated mixing of wall and plasma material during the implosion, resulting for example from Rayleigh-Taylor instabilities in the liner

- plasma thermal transport during the implosion

- diagnostic methods under conditions of energetic implosions

We recommend a multi-institutional MTF research program to address these important experimental and theoretical questions. In addition, studies are needed on how MTF would best be utilized for electricity generation or other applications. Qualitatively the intrinsically pulsed nature of MTF makes it similar to ICF in its potential application. Early studies of an electrical power plant based on liner technology [Krakowski 78] indicated the basic feasibility of a pulsed liner-driven system, and identified numerous technology issues that must be solved.

An intriguing more recent study of power generation using MHD conversion of fusion energy [Logan 93] indicates that MTF is well matched to the requirements of an MHD conversion system. The energy from 14-MeV neutrons would be used to vaporize and heat a lithium-containing blanket to 1 or $2 \mathrm{eV}$. Then MHD conversion gives higher efficiency 
and a greatly reduced balance of plant cost leading to considerably less expensive electricity compared with conventional MFE reactor concepts.

\section{Conclusiọns}

We briefly reviewed some very elementary features of all the standard fusion approaches. The main assumptions were that the fusion fuel is deuterium and tritium with a $10 \mathrm{keV}$ Maxwellian velocity distribution. We emphasized the variation of quantities with fuel density and observed that the system size becomes small, and energy requirements are much reduced, when fuel density is made considerably larger than in conventional MFE systems. This general conclusion, which has been noted by many researchers in the past, warrants renewed attention today as the fusion program restructures itself within today's budget limitations.

The reasons for embarking on an MTF research effort at the present time are several:

- The cost of development for fusion has become a major consideration in recent years, and MTF appears to offer advantages compared with MFE and ICF.

- The pulsed power facilities of Defense Programs, both DOE and DOD, are remarkably well matched to what is needed to investigate MTF.

- In the twenty years since MTF-like concepts were last seriously pursued in the United States, the theoretical understanding and experimental methods of plasma science as well as the technology of high-energy liner implosions have advanced significantly.

The interesting regime ve call Magnetized Target Fusion occurs at fuel density of about $10^{20} \mathrm{~cm}^{-3}$. The MTF regime may be an optimum in the sense of using the maximum possible magnetic field for insulation of the fuel, and thus the smallest possible system size without going to the extreme density of ICF. This new thrust in fusion research has the potential to achieve the lowest possible development cost.

We believe that the arguments presented here are robust in nature and give a valid basis for recommending a new research thrust in magnetic fusion energy. Given the global importance of long-term energy R\&D, adding MTF as a new complementary element to MFE and ICF in the portfolio of fusion approaches seems well justified.

\section{Acknowledgements}

We appreciate receiving encouragement to examine MTF from John Browne, Steve Younger, and Al Sattelberger. We also thank our colleagues Carl Ekdahl, Bob Reinovsky, and others in the pulsed power community at Los Alamos for providing expert advice on liner technology.

\section{References}

Bangerter 97. R. Bangerter, "The U. S. Heavy Ion Fusion Program," $12^{\text {th }}$ International Symposium on Heavy Ion Inertial Fusion and Workshop on Atomic Physics, Heidelberg, Germany,September 22-27, 1997.

Boozer 83. A. H. Boozer, Phys. Fluids 26, 496 (1983). 
Chang 78. J. Chang, M. M. Widner, A. V. Farnsworth, R. J. Leeper, T. S. Prevender, L. Baker, J. N. Olsen, in High Power Electron and Ion Beam Research and Technology (Proc. $2^{\text {nd }}$ Int. Top. Conf. Ithaca, NY, 1977) Vol.1, Cornell University (1978) 195.

Chernyshev 97. :V. K. Chernyshev, "Study of Condensed High Energy Liner Compression," $11^{\text {th }}$ International Pulsed Power Conference, Baltimore, Maryland, June 1997, and other papers of this conference.

Degnan 96. J. H. Degnan, S. K. Coffey, D. G. Gale, J. D. Graham, t. al., "Solid spherical and cylindrical shell z-pinches used to compress hot hydrogen working fluid," 1996 IEEE international conference on plasma science, June 3-5, Boston (1996), 43. Also, J. H. Degnan, F. M. Lehr, J. D. Beason, G. P. Baca, D. E. Bell, A. L. Chesley, S. K Coffey, D. Dietz, D. B. Dunlap, S. E. Englert, T. J. Englert, D. G. Gale, J. D. Graham, J. J. Havranek, C. D. Holmberg, T. W. Hussey, R. A. Lewis, C. A. Outten, R. E. Peterkin, D. W. Price, N. F. Roderick, E. L. Ruden, U. Shumlak, G. A. Smith, P. J. Turchi, Phys. Rev. Ltrs. 74, 98 (1995).

Gerwin 79. R. A. Gerwin, R. C. Malone, "Adiabatic Plasma Heating and Fusion-Energy Production by a Compressible Fast Liner," Nucl. Fusion 19(2), 155 (1979).

Gross 76. B. Feinberg, "An experimental study of hot plasma in contact with a cold wall," Plasma Physics 18, 265 (1976), =

Hammer 91. J. H. Hammer, J. L. Eddleman, C. W. Hartman, H. S. McLean, A. W. Molvig, "Experimental demonstration of compact torus compression and acceleration," Phys Fluids B 3, 2236 (1991).

Holdren 95. The U.S. Program of Fusion Energy Research and Development, The President's Committee of Advisors on Science and Technology (PCAST), "Report of the Fusion Review Panel," J. Holdren, Chairman, (July 1995).

Jarboe 94. T.R. Jarboe, "Review of spheromak research," Plasma Phys. Control. Fusion 36, 945 (1994).

Kadomtsev 92. B. B. Kadomtsev, Tokamak Plasma: A Complex Physical System, (Institute of Physics Publishing, Bristol and Philadelphia, 1992).

Krakowskd 78. R. W. Moses, R. A. Krakowski, R. L. Mille, "A conceptual design of the fastliner reactor (FLR) for fusion power," Los Alamos Scientific Laboratory informal report, LA7686-MS (1979) [available on LANL library web site].

Kurtmullaev 1980. S. G. Alikhanov, V. P. Bakhtin, A. G. Es'kov, R. Kh. Kurtmullaev, V. N. Semenov, E. F. Strizhov, N. P. Kozlov, V. I. Khvesyuk, A. V. Yaminskij, "Three-dimensional Plasma Compression in a Z-Pinch Liner System - Transport and Compression of a Compact Torus by a Quasi-Spherical Liner, $8^{\text {th }}$ LAEA Fusion Energy Conference III, 319 (1982).

Lindemuth 83. I. R. Lindemuth, R. C. Kirkpatrick, "Parameter space for magnetized fuel targets in inertial confinement fusion," Nuclear Fusion 23(3), (1983).

Lindemuth 95. I. R. Lindemuth, et.al., "Target Plasma Formation for Magnetic Compression/Magnetized Target Fusion," Phys. Rev. Ltrs. 75(10), 1953-1956 (September 1995).

Lindemuth 96. I. Lindemuth, C. Ekdahl, R. Kirkpatrick, R. Reinovsky, R. Siemon, P. Sheehey, F. Wysocki, V. Chernyshev, V. Mokhov, A. Demin, S. Garanin, V. Korchagin, I. Morozov, V. Yakubov, J. Eddleman, J. Hammer, D. Ryutov, A. Toor, D. McDaniel, J. Degnan, G. Kiuttu, R. Peterkin, Jr., "Magnetic Compression/ Magnetized Target Fusion (MAGO/MTF): A Marriage of 
Inertial and Magnetic Confinement," $16^{\text {th }}$ IAEA Fusion Energy Conference, Montreal, Canada, October 7-11, 1996.

Lindl 95. J. Lindl, "Development of the Indirect-Drive Approach to Inertial Confinement Fusion and the Target Physics Basis for Ignition and Gain," Phys. of Plasmas 2(11), 3933-4024

(November 1995).

Logan 93. B. G. Logan, "Inertial Fusion Reactors using Compact Fusion Advanced Rankine (CFARII) MHD Conversion," Fusion Engineering and Design 22, 151-192 (1993).

Pavlovskil 96. Paper at Megagauss V (1996)

Robson 76. A. E. Robson, P. J. Turchi, "NRL Linus Program," Pulsed high beta plasmas ( $3^{\text {rd }}$ topical conference on pulsed high beta plasmas, Pergamon Press, Oxford, 1976), 477. Also: P. J. Turchi, Review of the NRL Liner Implosion Programme, Megagauss Physics and Technology (Plenum Press, New York, 1980), 375

Ryutov 96. R. P. Drake, J. H. Hammer, C. W. Hartman, L. J. Perkins, D. D. Ryutov, "Submegajoule Liner Implosion of a Closed Field Line Configuration," Fusion Tech. 30, 310-325 (1996).

Schoenberg 98. K. Schoenberg et. al., "Application of the ATLAS facility to MTF Experiments," to be published.

Sheehey 89. P. Sheehey, "Computational Modeling of Wall-supported Dense Z-Pinches," Proc. of Second International Conference on Dense Z-Pinches, Laguna Beach, California, April 26-28, 1989.

Sheffield 94. J. Sheffield, The physics of magnetic fusion reactors, Reviews of Modern Physics 66(3), 1015 (1994).

Sherwood 81. A. R. Sherwood and F. L. Ribe, "Fast-Liner-Compression Fusion Systems," Fusion 1, Part B, $59-78$ (1981).

Siemon 97. R. E. Siemon, "Magnetized Target Fusion - A High-Density Pulsed-Power Approach to Fusion," Los Alamos National Laboratory talk LA-UR-97-764, presented at the Innovative Confinement Concepts Workshop, Marina del Rey, California, March 3-6, 1997.

Spitzer 62. L. Spitzer, Physics of Fully Ionized Gases (Interscience Publishers, New York \& London, 1962), p. 47.

Trainor 97. J. Trainor, et. al., "Overview of the Atlas Project," Proc. of 11th IEEE International Pulsed Power Conference (Baltimore, MD, June 29- July 2, 1997, to be published).

Tuszewski 88. M. Tuszewski, "Field Reversed Configuration," Nuclear Fusion 28(11), 2033-2092 (1988).

Vekshteln 90. G. E. Vekshtein, "Magnetothermal Processes in Dense Plasma," Rev. Plas. Physics 15, 1 (1990).

Wysocki 97. F. J. Wysocki, R. E. Chrien, G. Idzorek, H. Oona, D. O. Whiteson, R. C. Kirkpatrick, I. R. Lindemuth, and P. T. Sheehey, "Progress With Developing A Target For Magnetized Target Fusion", Proc. of 11 th IEEE International Pulsed Power Conference (Baltimore, MD, June 29- July 2, 1997, to be published). 


\section{APPENDIX B. Other MTF Target Plasmas}

In this appendix we briefly review several previous or ongoing MTF target formation efforts:

- Sandia phi-target [Lindemuth81],

- Russian (VNIIEF) MAGO system [Lindemuth95],

- z-pinches utilizing either static gas-fill or exploding fibers [Wysocki97]

- the spheromak type of CT.

\section{Phi-Target}

The phi-target was investigated briefly at Sandia National Laboratory around 1977, during which approximately 39 experiments (discharges) were conducted (Fig. B1). These experiments were unique in that both target-plasma generation and target implosion were performed in the same experiment utilizing a small relativistic electron-beam machine (single beam from the REHYD device). The target package consisted of a 3-mm-diameter spherical shell with a $0.1-\mathrm{mm}$-thick glass wall or a $0.3-\mathrm{mm}$-thick polystyrene wall. The capsule had small metal end-caps at both poles. One end-cap was connected to the anode of the electron-beam machine. The other end-cap was connected to a collector-plate outside the capsule, which intercepted the low intensity electron pre-pulse. In some experiments, the interior of the capsule was filled with approximately 100 tort of DT gas, while in other experiments, the interior contained a deuterated polyethylene (CD2) wire 25 to $50 \mathrm{~mm}$ in diameter along the symmetry axis and connecting the two metal end-caps.

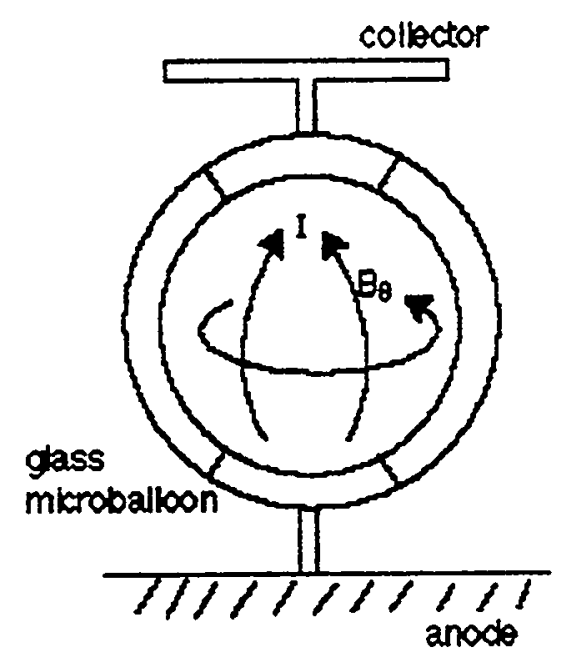

Fig B1. Phi-Target geometry.

The pre-pulse current of electrons rose to between 5 and $15 \mathrm{kA}$ in a ramp over a period of $1 \mu \mathrm{s}$. This current was collected by the collector-plate and gave rise to a discharge inside the capsule, which thus preheated and magnetized the target plasma. Two-dimensional MHD modeling indicates that the peak temperature in this target plasma was $21 \mathrm{eV}$, while the average ion density was $6 \times 10^{18} \mathrm{~cm}^{-3}$ [Lindemuth81].

The main pulse of $1 \mathrm{MeV}$ electrons had a current of $250 \mathrm{kA}$ and a FWHM of 100 ns. These electrons passed through the collector plate and deposited roughly $4 \mathrm{~kJ}$ directly into the wall of the capsule, creating an exploding-pusher implosion of the target plasma. The pusher velocity reached $4 \mathrm{~cm} / \mu \mathrm{s}$ giving an 
implosion time of roughly 40 ns. Diagnostics included Ag activation, neutron time-of-flight, and optical streak data for pusher motion.

Two-dimensional MHD modeling indicates a peak temperature of $362 \mathrm{eV}$ at a radial compression ratio of 15 , an average density of $1.7 \times 10^{22} \mathrm{~cm}^{-3}$, and an expected neutron yield of $10^{6}$. Neutron yields in the range of $5 \times 10^{6}$ to $3 \times 10^{7}$ were observed in 7 of the 15 experiments where the target package was "complete." For the remaining roughly 24 experiments, some aspect of the target package was purposefully "damaged," i.e., half the spherical shell was missing, or there was no gas or polyethylene wire present to form the target plasma, or the collector-plate was missing, or the electron pre-pulse was effectively eliminated. In every one of these "damaged" experiments, the neutron yield was less than the detection threshold of $1 \times 10^{6}$. Since only "complete" target packages produced a neutron yield, there is reasonable evidence that the neutron production is truly thermonuclear and the system is behaving reasonably like the 2-D MHD calculations.

An interesting aspect of these phi-target experiments is the very small energy content in the target system, due to the small size of the target and relatively low intensity of the electron-beam driver. After the preheat phase, the target-plasma thermal energy content is less than $0.4 \mathrm{~J}$. The calculated work done by the pusher at a radial compression of 15 is only $19.5 \mathrm{~J}$, of which $7.7 \mathrm{~J}$ remained as an increase in plasma thermal energy, giving a total of $8.1 \mathrm{~J}$. The other $11.8 \mathrm{~J}$ is lost to radiation and thermal conduction. For comparison, a typical Nova ICF capsule at peak compression has roughly $600 \mathrm{~J}$ of plasma thermal energy, 75 times as much as the phi-target experiments.

\section{MAGO}

While the phi-target experiments represent a low-end in energy content, the Russian MAGO experiments [Lindemuth95] represents the present high-end of energy content for MTF target plasmas. The MAGO device, (Fig. B2) is typically powered by a high-explosive driven electrical generator (EMG) that produces a slow rising current to roughly 2.7 MA, followed by a fast rising current to approximately 7-8 MA in 2-3 $\mu$ s.

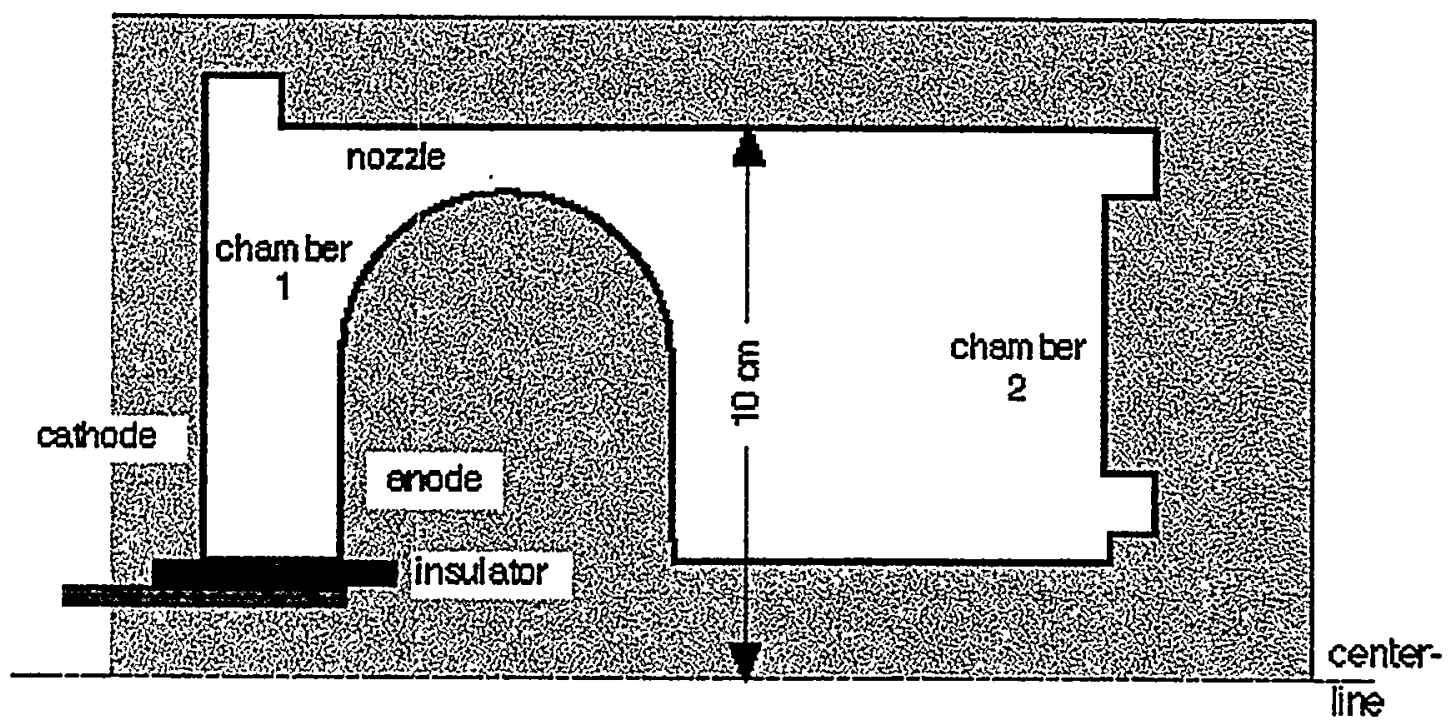

Fig. B2. MAGO chamber. 
The typical plasma chamber is cylindrical with a $10 \mathrm{~cm}$ radius, roughly $8 \mathrm{~cm}$ in length, and has a conducting $1 \mathrm{~cm}$ radius center-rod that carries the slow rising $2.7 \mathrm{MA}$ of current. The fast rising current drives a complicated dynamic plasma motion in which an inverse-pinch plasma starting in a neighboring chamber is driven through a nozzle region connecting the two chambers at the outside diameter.

Personnel from LANL have collaborated with VNIIEF personnel in diagnosing the resulting target plasma from four experiments, two performed at VNIIEF and two performed at LANL during the years 1994 to 1995 . In summary, over $10^{13} \mathrm{D}$-T neutrons are produced as a result of forming the target plasma, plasma density is approximately $6 \times 10^{17} \mathrm{~cm}^{-3}$, and the plasma temperature reaches over $200 \mathrm{eV}$ for a period of 2-3 $\mu \mathrm{s}$. Unfortunately, the harsh environment for the diagnostics has so far prevented a measurement of the plasma cooling time, and the plasma temperature after the initial hot period is experimentally unknown at this time. The peak plasma thermal energy content is estimated to be at least $50 \mathrm{~kJ}$. More recently, VNIIEF has begun experiments with smaller chambers and smaller EMG drivers that are cheaper and allow more frequent experiments. Also VNIIEF now has a capacitor bank system operational that can deliver roughly $3 \mathrm{MA}$ of current to a MAGO chamber, allowing even more frequent experiments. LANL personnel are expected to make measurements on these systems in the summer of 1998. At this time, the MAGO target plasma has not been imploded.

\section{Z-Pinch Target}

Target plasma experiments have been performed on the Colt facility at LANL since 1996 using a zdirected current through plasma contained in a conducting chamber. Colt can produce a drive current of 2 MA rising in $2.5 \mu \mathrm{s}$. Experiments have been performed where the plasma chamber and connecting power flow region are statically filled with deuterium gas prior to the discharge. In addition, an exploding fiber approach is being studied, where the discharge current is driven through a cryogenically frozen $200 \mu \mathrm{m}$ diameter deuterium fiber. The initial small diameter plasma will go unstable and "explode" and heat from instability heating. In less than $1 \mu \mathrm{s}$, the plasma expands to the conducting wall and becomes wall stabilized. By the time of peak drive current, 2-D MHD calculations indicate a peak plasma temperature of up to $350 \mathrm{eV}$ is possible, at an average density of roughly $1 \times 10^{18} \mathrm{~cm}^{-3}$. Data from static gas-fill discharges show that the plasma density in the plasma chamber rises to over $1 \times 10^{18} \mathrm{~cm}^{-3}$. Magnetic pickup probes at the wall and fast framing camera pictures of the plasma indicate that the plasma is quiescent after an initial roughly $0.4 \mu \mathrm{s}$ unstable period. An array of filtered soft $\mathrm{x}$-ray diodes indicates that the hot plasma lasts roughly $6 \mu \mathrm{s}$. While not conclusive, the $\mathrm{x}$-ray diode data indicates a peak temperature of roughly $70 \mathrm{eV}$. The performance of these static gas-fill discharges is limited by the fraction of the drive current actually delivered to the plasma chamber region. Because the power-feed region is also gas filled, $60-80 \%$ of the drive current remains in this region. It is hoped that this problem will be solved with the cryogenic fiber approach.

\section{Spheromak Target}

The spheromak is another form of compact toroid that could be used as an MTF target plasma. In the context of "traditional" MFE, the spheromak has been studied beginning around 1979, and is reasonably well understood [Jarboe94]. We have considered the possibility of extending the operating parameters to achieve conditions relevant to MTF. The spheromak target plasma would be contained in a cylindrical conducting metal containment region with a $3-\mathrm{cm}$ radius and a $3-\mathrm{cm}$ height. The expected plasma parameters are based on scaling results from the LANL CTX spheromaks in both $61 \mathrm{~cm}$ radius $(62 \mathrm{~cm}$ height) and $28 \mathrm{~cm}$ radius ( $28 \mathrm{~cm}$ height) containment regions. Plasma temperature of $130 \mathrm{eV}$ and plasma 
density of $4 \times 10^{13} \mathrm{~cm}^{-3}$ were obtained from the $61 \mathrm{~cm}$ CTX spheromak [Wysocki90], and temperature and density of $350 \mathrm{eV}$ and $4 \times 10^{14} \mathrm{~cm}^{-3}$ from the $28 \mathrm{~cm}$ CTX spheromak [Jarboe90]. Based on these data, we estimate that a spheromak with an initial toroidal plasma current of $2.8 \mathrm{MA}$ could ohmically heat a plasma with a density of $3 \times 10^{17} \mathrm{~cm}^{-3}$ to roughly $400 \mathrm{eV}$ in 1-3 $\mu$. The e-folding decay time of the resulting hot uncompressed plasma is estimated at 5-16 $\mu \mathrm{s}$. A spheromak MTF target like that described here is compatible with a quasi-spherical liner implosion like those performed at the Shiva-Star facility at the AFRL [Degnan95].

A spheromak of this size with a toroidal plasma current of 2.8 MA has an initial magnetic energy content of $26 \mathrm{~kJ}$. Previous CTX spheromaks had this level of magnetic energy content, but were much larger in size. We have considered the required spheromak injector parameters needed to generate an MTF relevant spheromak. Based on previous data [Barnes86, Barnes90], a magnetized plasma gun with an inner electrode radius of $1.34 \mathrm{~cm}$ and an outer electrode radius of $2.14 \mathrm{~cm}$, coupled with a gun bias flux of 4.9 $\mathrm{mWb}$, a gun current of $1.3 \mathrm{MA}$, and a gun voltage of $21 \mathrm{kV}$ is expected to generate the spheromak parameters desired.

For further information on the possibilities and parameter space of a spheromak MTF target, see the following URL: http://fusionenergy.lanl.gov/

Spheromak References

[Jarboe94] T. R. Jarboe, Plasma Phys. Control. Fusion 36, 945 (1994), and references within.

[Wysocki90] F. J. Wysocki, J. C. Fernández, I. Henins, T. R. Jarbo, and G. J. Marklin, Phys. Rev. Lett. 65 (1), 40 (1990).

[Jarboe90] T. R. Jarboe, F. J. Wysocki, J. C. Fernández, I. Henins, and G. J. Marklin, Phys. Fluids B 2 (6), 1342 (1990).

[Degnan95] J. H. Degnan, F. M. Lehr, J. D. Beason, G. P. Baca, D. E. Bell, A. L. Chesley, S. K. Coffey, D. Dietz, D. B. Dunlap, S. E. Englert, T. J. Englert, D. G. Gale, J. D. Graham, J. J. Havranek, C. D. Holmberg, T. W. Hussey, R. A. Lewis, C. A. Outten, R. E. Peterkin, Jr., D. W. Price, N. F. Roderick, E. L. Ruden, U. Shumlak, G. A. Smith, and P. J. Turchi, Phys. Rev. Lett. 74 (1), 98 (1995).

[Barnes86] C. W. Barnes, J. C. Fernández, I. Henins, H. W. Hoida, T. R. Jarboe, S. O. Knox, G. J. Marklin, and K. F. McKenna, Phys. Fluids 29(10), 3415 (1986).

[Barnes90] C. W. Barnes, T. R. Jarboe, G. J. Marklin, S. O. Knox, and I. Henins, Phys. Fluids B2(8), 1871 (1990). 


\section{APPENDIX C: Theory of Wall-Plasma Interactions}

This appendix discusses physical considerations important to confining a plasma directly by the liner wall. The first section (by D. Ryutov) gives an overview of the problem, and the second section (by P. Parks) offers another perspective on the issues that are likely to arise during MTF implosions.

\section{The physics of a wall confinement of a plasma with $\beta \gg 1$.}

In this section, we present a brief summary of the theory of the wall confinement. A complete description of the problem would have required not a few pages but a few hundreds of pages - a task that goes far beyond the scope of the present document. Here we are simply going to identify some key phenomena governing the wall confinement.

Let a hot dense plasma with initially uniform magnetic field embedded into it get in contact with a material wall parallel to the magnetic field. Let the wall be perfectly conducting (we will discuss variations caused by the finite wall resistivity later). Assume first that the initial plasma beta is not very high, $\beta<10-20$. When the cooling of the plasma adjacent to the walls begins (because of the heat losses to the wall), compression of this layers occurs and plasma starts to flow towards the wall, advecting the magnetic field. The magnetic pressure near the wall grows and reaches the value approximately equal to the plasma pressure in the center. The compression ratio of the magnetic field and the plasma is

$\sim \sqrt{\beta}$. On can mention parenthetically that the cold dense plasma filling this zone (and having the pressure much less than magnetic pressure) may have a favorable effect on the stability of the system.

The confinement in this mode remains similar to a traditional magnetic confinement, just the strong magnetic field near the walls is created by the plasma itself. Let's denote by $\Delta$ the thickness of the layer with a high magnetic field. According to our previous discussion, $\Delta \sim R / \sqrt{\beta}$, where $R$ is a plasma radius. In the simplest model where the thermal conductivity $k$ is a constant, the heat flux through the layer is $\sim 2 \pi R K T / \Delta$, whereas the heat content per a unit length of a plasma column is $\pi R^{2} n T$. This yields the following estimate for the confinement time:

$$
\tau \sim \frac{R^{2}}{\chi \sqrt{\beta}}
$$

where $\chi \sim K / n$ is the thermal diffusivity. In a general case, the dependence of the confinement time on $\beta$ is determined by the dependence of the thermal conductivity on the plasma parameters.

With $\chi$ increasing, new elements in the picture emerge. First, the thickness of a transition layer may become comparable with the skin-depth $\delta$. This effect becomes important at

$$
\beta>(R / \delta)^{2},
$$

with the skin depth evaluated for the confinement time (1). When the inequality (2) is satisfied, the faster advection of the plasma with the embedded magnetic field will occur, and the confinement time will decrease compared to the estimate (2).

Second, at higher betas, the plasma radiation from the dense layer may become important (because the radiation losses per unit plasma volume scale as $n^{2}$ ). The natural boundary for this effect to become 
important can be evaluated from the following considerations. The characteristic density and the temperature in a dense layer scale as $\sqrt{\beta}$ and $1 / \sqrt{\beta}$ with respect to their values in the plasma core. Assuming that radiation losses scale as an inverse square root of the temperature, one finds that the radiation power per unit volume of the dense layer is

$$
q_{\text {cole }} \beta^{5 / 4} q \text {, }
$$

where $q$ is the same quantity for the plasma core. Radiation heat losses from the dense layer, per unit length of the plasma column, are: $2 \pi R \Delta q_{\text {cols }}$. Imposing the constraint that the radiative losses are smaller than conductive losses, $\pi R^{2} n T / \tau$, with $\tau$ as in estimate (1), one finds that radiative losses are insignificant if

$$
\tau_{\text {rad }}>\tau \beta^{1 / 4}
$$

where $\tau_{\text {rad }}$ is the radiative cooling time for the parameters of a plasma core (approximately 30 times longer than the Lawson time for $Q-1)$. At higher betas, the confinement time will be smaller than according to estimate (1).

At very high betas and a poorly conducting wall, a regime where the diffusive scaling is replaced by the advective scaling sets in: the radiative cooling, in combination with the leak of the magnetic field into the wall, causes continuous plasma flow toward the wall, where the DT plasma cooled to temperatures $\sim 0.5$ electronvolt becomes, in a sense, a part of the wall. In this regime, the confinement time depends on $R$ not quadratically but only linearly.

An interesting feature of the wall confinement of a high-beta plasma is that the transitional layer is virtually impermeable to the impurities: first, the thermal force in a high beta plasma is directed in such a way as to repel impurities from the hotter plasma; second, there is a plasma flow towards the walls. It goes without saying that, at the plasma parameters of interest for MTF, the direct penetration of neutral atoms from the wall is limited to distances of a few micron.

The actual value of the transport coefficients in the magnetized region, where both $v / \omega_{C_{i}}$ and $v / \omega_{C_{i}}$ are much less than 1 , is almost certainly determined by drift-type microinstabilities. They can hardly result in the transport coefficients exceeding the Bohm diffusion coefficient. In the case of a high enough density, Bohm losses are compatible with the required confinement time, and the need in the studies of the microturbulence is minimal. In the case of lower densities, one would have to assess the issues of drift instabilities in a $\beta>1$ plasma - an issue that had not yet been studied at any depth.

So far, we have been discussing a situation where the field lines are straight. This is a good approximation in a narrow near-wall region. However, in the bulk plasma one will have to consider the presence of curved magnetic field lines and of (possible) MHD instabilities associated with the curvature of the magnetic field. A concern with regard to these instabilities is that they may set in large-scale convective motions that would cause heat losses at a time scale that is shorter than even the Bohm time scale.

Somewhat paradoxically, the high-beta plasma, whose pressure is almost uniform across the flux surfaces (at least in the hot region where the local beta is very large) is more stable with respect to the curvaturedriven modes than its low-beta counterpart. The reason for this is that the magnetic field has too small energy to be able to cause compression or rarefaction of the plasma with $p=$ const $>>B^{2} / 2 \mu$ (the perturbation of the plasma thermal energy would become prohibitively high). Therefore, only the 
perturbations with $\nabla \cdot \xi=0$ (with $\xi$ being a standard MHD displacement vector) remain admissible. This imposes an additional constraint on the perturbations allowed to compete in the minimization of the potential energy and thereby improves MHD stability. Possible residual MHD instability is additionally suppressed by a strong longitudinal ion viscosity, which is not suppressed by the plasma magnetization. Still, so far there is no proof that there exists closed-field-line configurations stable with respect to all classes of the MHD perturbations. Therefore, considerable efforts in theory and experiment are needed to clarify the situation.

An additional element that may affect the mix of the liner and the hot fusion plasma, is the RayleighTaylor instability of the inner surface of the liner during the deceleration phase near the point of the maximum compression.

Let us first discuss the situation of moderate central betas; in such situations, a magnetic cushion with beta less than one is formed near the walls. For the perturbations with the length-scale smaller than the thickness $\Delta$ of this cushion, the situation will not differ from a so called magnetic Rayleigh-Taylor instability studied in great detail in conjunction with implosions of fast liners. A huge body of theoretical and experimental information is presently available; based on this knowledge, we will certainly be able to evaluate in what parameter domain this instability is not too harmful. Perturbations with the scale-length longer than $\Delta$ are considerably slower and are of less concern.

Consider now the situation where beta is much greater than one until the liner surface (this, as has been pointed out, corresponds to very high central betas). In this case, one has a situation of a heavy liner decelerated by a medium of a very low mass density and essentially isorropic pressure (anisotropy of the magnetic stress tensor is small). This is also a system studied in great detail. Using the available information, we will be able relatively quickly identify an acceptable parameter domain (if existent) for implosions of very high beta loads.

\section{Can Magneto-Inertial Fusion Plasmas Overcome Wall Effects?}

One of the foremost scientific problems in Magneto-Inertial or Magnetic Target Fusion (MTF) is the role of plasma-wall effects on the thermal losses from the plasma core. A general semi-quantitative view of plasma-wall effects and outstanding problems is offered here. There are two fundamental heat loss mechanisms, and they are tightly coupled in the case of highdensity MTF compressions. First, there is a steepening of the edge temperature profile by mere contact with the imploding liner, and second there is impurity production caused by bremsstrahlung heating of the liner material and subsequent mixing of vaporized liner material by Rayleigh-Taylor (RT) interchange motions. Beneficial exemplifications of interchange mixing are noted, and may be employed usefully in connection with a scenario for enhancing target gain by refueling the burning MTF plasma [Barnes, 1997].

\section{(1) Effect of Temperature Gradients near Wall Confined Plasmas}

Insight on the effect of temperature profile steepening in the proximity of a wall can be gained from the point of view of a prototypical model of a seminfinite, uniform plasma which is brought into sudden contact with a solid wall [Vekshtein, 1990]. The plasma is hot, high-beta $\beta=\beta_{0} \gg 1$, and highly magnetized $\omega_{\mathrm{ci}} \tau_{\mathrm{i}} \gg 1$, and it is assumed to be supported by the inertia of the wall, so initially $\nabla p=0$. During readjustment of the plasma profiles on the slow diffusive 
time scale, quasi-equilibrium $\nabla\left(p+B^{2} / 2\right)=0$ is consistently maintained across the plasma. Thus, as a cool plasma boundary layer advances into the hot interior, it will naturally be accompanied by a density increase and an increased particle accumulation in the layer. Even before the cool layer has reached the interior region, the interior region knows about the cool layer by magnetosonic wave propagation, and thus a slow outward flow of plasma to the wall develops in order to satisfy particle continuity. The hot region effectively loses heat by adiabatic expansion: particles and magnetic flux being carried to the wall at the common $E \times B$ flow speed.

In MTF applications, the wall may act more like an insulator; as, for instance, in the case of a metallic liner undergoing heating to supercritical temperatures without significant ionization, or in the case where the inner surface of the metal liner is coated with a DT fuel layer. In this case the the B-field at the wall remaines constant in time at its initial value, and the outward flow speed scales as $v_{\text {outflow }}=0.3\left(\omega_{\mathrm{ci}} \tau_{\mathrm{i}}\right)^{1 / 2} \chi_{\mathrm{B}}^{1 / 2} t^{-1 / 2}$, where $\chi_{\mathrm{B}}=T / e B$ is the Bohm diffusion coefficient. Using some reference parameters for the MTF plasma at the time of peak compression $(t \sim 10 \mu \mathrm{s})$, the outflow velocity can exceed $\sim 1 \mathrm{~cm} / \mu \mathrm{s}$. This velocity is comparable to currently envisioned liner velocities. This model may suggest that the driver technology may have to be pushed furthur to achieve even higher liner velocities, for otherwise adiabatic heating during liner implosion may be counteracted by wall-induced expansion cooling. Note that this outflow velocity is equivalent to an effective thermal diffusivity in the hot plasma which turns out to be higher than the good classical thermal diffusivity by a large factor, $0.3 \omega_{\mathrm{ci}} \tau_{\mathrm{i}}$, i.e. for a high- $\beta$ plasma, the effective thermal conductivity becomes Bohm scaling: $\chi_{\text {eff }}=0.3 \chi_{\mathrm{B}}=0.3 T / e B$.

-- Caution: Drake et al, may have incorretly used a Bohm coefficient 48 times smaller than the above 0.3 value in their Fusion Technology 1996 paper, Eq (31), I do not know what coefficient Lindemuth uses in his 1-D simulations. ]

The energy confinement improves to some extent if the wall acts like a good conductor. Essentially, quasi-equilibrium could then be satisfied at lower flow rates by piling up magnetic field, rather than particles, in the boundary layer. For typical MTF compressed plasmas, the Vekshtein model suggests a well magnetized $\beta \sim 1$ boundary layer will be formed. In this case only a modest increase in the effective thermal diffusivity from the good classical value results, $\chi_{\text {eff }}=\beta_{0}^{1 / 4} \chi_{\mathrm{cl}}$. Note that in MTF plasmas the central beta values are typically $\beta_{0} \sim 10$.at peak compression.

Although the ideal model has some deficiencies, it reveals how profoundly different the effect of a wall has on heat transport in a high-pressure $\beta \gg>1$ wall-supported plasma as compared with its effect on heat transport in the conventional $\beta<1$ magnetically confined plasma. We will need to generalize this model by including finite plasma volumes with different geometries ( cylindrical, spherical, elliptical, time-dependent compression, and initial low-beta edge regions which exist in many preformed magnetic configurations considered for MTF. We will also need to include wall generated impurity effects in the models, and finally effects due to the specific magnetic topology. For example, in some MTF candidates with topological open field lines on the outer surface, such as the FRC and the spheromak, wall heating by cross-field 
heat conduction can be limited by rapid parallel heat transport. In particular, most plasma objects formed just before compression are not initially held together by wall inertia, as assumed in the Vekshtein model. Confinement is initially provided by a surrounding magnetic field with equilibruim dictated by $\nabla_{\perp}\left(p+B^{2} / 2\right)-B^{2} \bar{k}=0$ where $\bar{\kappa}$ is the field line curvature.

Some insight can be gained into how the compression of real objects changes their profiles and gradients and how this relates to the heat transport near the liner. Consider a two-region initial configuration consisting of a medium-pressure core region with modest beta, $\beta=\beta_{0}$, surrounded by a low-pressure mantle with very low beta $\beta=\beta_{1}$, as in the case of an FRC configuration. A self-similar 2D shape-preserving compression is assumed in this exercise. The initial radius of the entire configuration, the initial liner radius, is $r=R_{l}$, and the initial coremantle radius is $r=R_{b}$. At peak compression, the final liner radius is $r=R_{Z^{*}}$, and the final coremantle radius is $r=R_{b^{*}}$. We now define a liner compression ratio compression ratio,

$$
A=\frac{R_{l}}{R_{l}}
$$

and we ask how this is related to the compression ratio of the core region

$$
A_{\mathrm{b}}=\frac{R_{\mathrm{b}}}{R_{\mathrm{b}^{*}}}
$$

Intuitively we anticipate that the core will be compressed to a lesser extent, $A_{\mathrm{b}}<A$. Since the core pressure rises faster than the mantle magnetic pressure a "ballooning" of the core-mantle boundary during the compression is anticipated somewhat analogous to a Vekshtein like flow. To determine the location of the core-mantle radius we assume total pressure balance across the entire configuration (neglecting field line curvature), magnetic flux conservation in each region, and the adiabatic law $p-$ volume ${ }^{-\gamma}$ for each region. The degradation of core compression may be characterized in terms of the parameter $\eta=A_{b} / A$, which is given implicitely by

$$
A=\left\{\frac{\eta^{(4-3 \gamma)}\left(x / \beta_{0}-\left(1-f_{0}^{2}\right)^{2}\left(\eta^{2}-f_{0}^{2}\right)^{-2} / \beta_{1}\right)}{\left(\frac{1-f_{0}^{3}}{\eta^{3}-f_{0}^{3}}\right)^{\gamma}-x} \cdot \mid\right]^{\frac{1}{3 \gamma-4}}
$$

in which $x=p_{0} / p_{1}=\left(1+\beta_{1}^{-1}\right) /\left(1+\beta_{0}^{-1}\right)$.is the initial pressure ratio, and $f_{0} \equiv R_{\mathrm{b}} / R_{l}$. Note that as $A$ approaches $\infty, \eta$ asymptotes to a minimum

$$
\eta_{\min }=\left(x^{-1 / \gamma}\left(1-f_{0}^{3}\right)+f_{0}^{3}\right)^{1 / 3}
$$


Take for example the numbers: $\beta_{0}=0.75, \beta_{1}=0.01$, and $x=43.29$. Choosing $t_{0}=$ separatrix radius/wall radius $=0.4$, we first obtain the lower limit $\eta_{\min }=0.5447$. To reach core fusion temperatures $\sim 10 \mathrm{keV}$, the typical core compression ratio needs to be $A_{\mathrm{b}}=10$. From Eq. (2), the liner compression ratio turns out to be $A=13.44$, and the parameter $\eta=0.748$. The final core beta is increased by a factor of ten $\beta_{0^{*}}=7.5$, and the beta in the mantle is increased to 0.115. Note that the fractional width of the mantle region has shrunken from an initial value of $1-f_{0}=0.6$ down to a final value of $1-f_{0} / \eta=0.465$.

The ballooning of the core plasma and the enhanced steepening of the pressure and temperature profiles caused by compression may excite resistivity-gradient driven turbulence, and enhance heat conduction losses ( see part 3). Because of the high-beta property of compressed MTF objects, it is hoped that ideal ballooning modes may be in the "second stable" regime due to the magnetic well effect. However, at high beta another issue may be with the kinetic ballooning modes, which have a resonance at a frequency near the velocity dependent ion magnetic drift frequency. Because the resonance effect is enhanced by the finite iontemperature-gradient $\eta_{\mathbf{i}}$ parameter [Hirose, 1996], MTF plasma profiles with steep temperature and density gradients of opposite sign may be particularly susceptible to these kinetic ITG modes. Typical growths rate times are $\sim 0.1$ Alfven times, and are thus comparable to the microsecond dwell times in MTF.

\section{(2) Impurities}

The ingestion of impuries from the wall into the plasma is profoundly altered in high pressure, $\beta \gg 1$ MTF plasmas. In low-beta tenuous plasma, the impurities emitted from the wall can freely cross the plasma boundary and bury a distance into the plasma determined only by their mean free path against ionization by the plasma electrons. In the case of MTF plasmas, the wall is intensely heated, but it may not freely vaporize and release impurities into the plasma, if the plasma pressure over the wall exceeds the vapor pressure at each moment during the compression. Of course, if the wall temperature reaches the critical temperature the wall material can penetrate the plasma by a mixing process initiated by the RT instability, which develops near the approach to final compression and the ensuing dwell period. On the other hand the RT mixing process will be influence by the outward flow of magnetic flux as we discussed, and this may limit the inward migration of the impurities. The nonlinear development of the RT mixing process with self consistent radiation cooling dynamics will be investigated with comprehensive analytical/numerical tools. Benchmarking of our models with experimental tests of interior cooling rates as inferred by liner compression speeds and spectroscopic measurements will be necessary to sort out the dominant physicall processes.

On the reverse side, the RT impurity mixing process may be exploited to our advantage to "refuel" the MTF plasma during the burn phase. If the interior liner surface were coated with a cryogenic DT layer, automatic mixing of cold fuel with the buring DT core during the RT unstable dwell period may prolong the duration of the burn and increase the target gain. This concept will be considered as part of our study on wall-plasma interactions.

Let us now consider liner heating in the case where the open-field-line sheath provides good thermal insulation. Neglecting ohmic heating, the dominant liner heating mechanism then 
becomes bremmstrahlung radiation. Even with some cross-field heat conduction to the surface the penetration of heat into the interior is much less efficient than radiation since the thermal diffusion distance into the liner $\sqrt{x^{t}}$ is usually quite small compared with the mean photon penetration depth from bremsstrahlung. Furthermore, any ablation layer formed by surface conduction heating will not expand away, as we will show. Liner heating during implosion is best described by combining an entropy equation of the form

$$
\frac{D s}{D t}=\frac{-\nabla \cdot \bar{q}}{\rho T}
$$

with the equation-of-state linking the temperature, $T$, and the density $\rho$ with the pressure $p$ and specific entropy $s$

$$
T=T(p, s), \rho=\rho(p, s,)
$$

To simplify the problem, we will assume that the liner stays thin compared to the radius of its inner surface, $R_{l}(t)$, so that the attenuation of bremsstrahlung heat flux with distance $x=r-R_{l}(t)$ into the liner material will be $q \approx q_{0} \exp (-x / \lambda)$, where the photon absorption mean free path is $\lambda=\lambda_{\infty} \rho_{c} / \rho$, and the "c" subscript refers to the properties of the condensed phase at $t=0$. By taking into account cylindrical convergence, the rate of entropy change for a Lagrangian fluid element $d x_{0}=\rho R_{l}(t) d x / \rho_{\mathrm{c}} R_{l}(0)$ which is initially at depth $x_{0}$ is given by

$$
\frac{\partial s\left(x_{0}, t\right)}{\partial t}=\frac{q_{0}}{T\left(x_{0}, t\right) p_{\mathrm{c}} \lambda_{\mathrm{c}}} \exp \left[-\frac{x_{0}}{\lambda_{\mathrm{c}}} \frac{R_{l}(0)}{R_{l}(t)}\right]
$$

Note that thickening of the liner by cylindrical convergence increases the photon attenuation, as manifest by the instantaneous compression ratio $R_{l}(0) / R_{l}(t)$ appearing in Eq. (6). The pressure distribution within the liner material is given by

$$
p_{l}\left(x_{0}, t\right)=p_{\mathrm{sur}}+\rho_{\mathrm{c} g} x_{0} R_{l}(0) / R_{l}(t)
$$

where the total plasma pressure, kinetic plus magnetic, exerted on the inner surface is $p_{\text {sur }}=p\left(1+\beta^{-1}\right) \approx p$, and $g$ is the instantaneous liner acceleration $(g>0$ for inward liner acceleration during the run-in phase, and $g<0$ for inward liner deceleration during the dwell period).

For simplicity let us neglect the change in the liner density during implosion. This may not be a bad assumption even at high temperatures because the liner is also subjected to high pressures. Then $T d s=C_{\mathrm{v}} d T$, and $\mathrm{Eq}$ (6) describes the temperature evolution for each Lagrangian fluid element, 


$$
\rho_{\mathrm{c}} C_{\mathrm{v}} \frac{\partial T\left(x_{0}, t\right)}{\partial t}=\frac{q_{0}}{\lambda_{\mathrm{c}}} \exp \left[-\frac{x_{0}}{\lambda_{\mathrm{c}}} \frac{R_{l}(0)}{R_{l}(t)}\right]
$$

If we next assume uniform plasma profiles, then the bremmstrahlung radiation power emitted per unit volume of a hydrogenic plasma is given by [Glasstone and Lovberg, 1960]

$$
P_{\text {brem }}=1.69 \times 10^{-26} n^{2} \theta^{1 / 2} \quad \text { Watts } / \mathrm{m}^{3}
$$

where $n\left(\mathrm{~cm}^{-3}\right), \theta(\mathrm{eV})$, are the plasma electron density and temperature, respectively. In a selfsimilar compression, the axial compression factor and the radial compression factor, $A(t)=R(0) / R(t)$, are the same, and for an adiabatic ideal gas $j=5 / 3$, so that $n=n(0) A^{3}$, and $\theta=\theta(0) A^{2}$. Utilizing these relations, Eq.(9) becomes

$$
P_{\mathrm{brem}}=1.69 \times 10^{-26} n(0)^{2} \theta(0)^{1 / 2} A(t)^{7} \text { Watts } / \mathrm{m}^{3}
$$

Since the plasma is optically thin to its own bremsstrahlung radiation, the heat flux falling on the inner liner surface is simply

$$
q_{0}=\alpha R_{l}(t) P_{\mathrm{Brem}} \quad \text { Watts } / \mathrm{m}^{2}
$$

where the shape coefficient $\alpha=$ (volume of the plasma) $/\left(R_{l}(t) \times\right.$ liner surface area) remains constant for a self-similar compression. Utilizing Kramers' photoionization formula, [Zel'dovich and Raizer, 1966], the photon mean free path in the liner material scales with the cube of the photon energy. Thus we can write for almost all candidate liner materials

$$
\lambda_{\mathrm{c}}=\lambda_{\mathrm{c} 10}\left(\frac{\theta(\mathrm{keV})}{10}\right)^{3}=\lambda_{\mathrm{c} 10}\left(\frac{\theta(0)(\mathrm{eV})}{10^{4}}\right)^{3} A(t)^{6}
$$

where $\lambda_{\mathrm{cl} 0}(\mathrm{~m})$ is the average mean free path of photons coming from a $10 \mathrm{keV}$ plasma. By combining Eqs.(8-11), we obtain finally

$$
\frac{\partial T\left(x_{0}, t\right)}{\partial t}=1.69 \times 10^{-14}\left(\frac{\alpha}{\rho_{c} C_{v} \lambda_{c 10}}\right) R(0) n(0)^{2} \theta(0)^{-5 / 2} \exp \left[-\frac{x_{0}}{\lambda_{c}} A(t)\right]
$$

Apart from the liner thickening effect, the compression factor drops out.

To minimize liner heating, an important consideration in the choice of liner materials is the $\sim Z^{3}$ dependence in the photon absorption crossection $\mu=1 / \rho_{c} \lambda_{c}$, for $Z<30$ [LLNL, 1969] 
which favors low- $Z$ materials. Let us then take, for example, a lithium liner with material properties $\rho_{\mathrm{c}} C_{\mathrm{v}}=2.1 \times 10^{\circ} \mathrm{J} / \mathrm{m}^{3} / \mathrm{K}$, and $\lambda_{\mathrm{c} 10}=0.08 \mathrm{~m}$. For nominal initial plasma conditions:

$$
R(0)=0.1 \mathrm{~m}, \theta(0)=100 \mathrm{eV}, p(0)=2 n(0) \theta(0)=6.4 \mathrm{MP}, \alpha=0.4 \text {, }
$$

the rate of temperature change at the inner liner surface $\left(x_{0}=0\right)$ is $1.78 \times 10^{9} \mathrm{~K} / \mathrm{s}$. If the compression time is $\sim 5 \mu \mathrm{s}$, the final liner temperature will be $8000 \mathrm{~K}$ which is well above critical temperature for lithium, $T_{\text {crit }}=3223 \mathrm{~K}$. Nevertheless, the liner pressure at the surface $p_{l}(0, t)=p_{\text {sur }} \approx p \approx p(0) A(t)^{5}$, appears to remain well above the vapor pressure of the lithium $p_{\text {vap }}=8.5 \times 10^{4} \exp \left(-2.17 \times 10^{4} / T\right) \mathrm{MPa}$. This means that impurity blowoff from the surface and volume vaporization (growth of vapor bubbles and disruption of the fluid ) can be avoided, and therefore liner impurity atoms cannot be brought into the plasma by liner heating alone.

It thus appears that the liner fluid can remain intact until the onset of the dwell period, $g<0$, when the RT instability and subsequent interchange mixing of the supercritical fluid with the core plasma can begin. The question of what fraction of liner material will undergo mixing depends on many details of the RT instability which need analytical and numerical formulation. Possible mitigating effects, such as line-tying on open field lines [Prater 74], the effect of a nonsteady $g$-force [Hattori 1986] and the fact that the pressure inside the liner falls off with depth, so that the liner density may also do the same, need to be taken into account. However it is clear that the depth of the heated layer as suggested by Eq. (13) will determine the maximim amount of impurity pollution.

Although the temperature of a lithium liner can approach $1 \mathrm{eV}$, it is still virtually unionized because of the very high liner pressures at peak compression, $p_{l} \sim 10^{6} \mathrm{MP}$. Since for all metals the supercritical fluid state is highly resistive, the liner may thus lose its good flux conserving property during the late phase of the compression. The resulting dissipation of magnetic flux near the wall would of course be compensated by an outward flow of flux from the plasma core to the wall. Such a Vekshtein-like flow could potentially degrade energy confinement.

In summary, the changing thermodynamic and fluid dynamic properties of the liner during compression and its significance on thermal losses from the plasma seem to be critical MTF issues. Analytical models will developed and incorporated in the numerical simulations in order to carry out systematic plasma-wall interaction studies.

\section{(3) Thermally Driven Convection Cells}

It is well known in tokamak plasmas that the plasma boundary has a high level of fluctuations. Some of the model candidate for edge turbulence are resistivity gradient driven turbulence and involve centering of the current density fluctuations about the mode rational surface $k \cdot B=0$, while the perturbed motions are growing on only on side of the surface. The essential features of the nonliner turbulent state involve a balance between the resistivity gradient drive and the parallel thermal conduction damping. When impurities are present there is a new drive term, namely the well-known thermal or "condensation" instability which results in the low temperature edge where the impurity line radiation rate increases as the temperature goes down. The thermal drive can couple with the resistivity gradient drive to enhance thermally driven convective cell turbulence. The coupling comes about because the perturbed potential appearing in Ohm's law due to the cross-field resistivity gradient also enters the temperature equation as a convective $\tilde{E} \times B$ perturbed flow. As shown by Thayer et al [Thayer 1987], the linear growth 
rates scales with impurity density, and non-linear diffusion coefficients are proportional to the impurity density squared. Hence, these modes may be particurily worrisome in the very highdensity wall confined MTF plasmas. Because MTF wall-plasma effects occur in an accelerated reference frame, the linear analysis of thermally driven convection cells would need to be modified by including an effective time-varying $g$ drive in the vorticity (momentum) equation. In addition, the previous analysis were based on an electrostatic approximation, with constant pressure $\mathrm{nT}$ assumed. As we have seen, however, even in the peripheral region of MTF plasmas, the plasma beta is not negligible, so in fact the stability analysis would have to be modified to include magnetic perturbations.

(4) References

Barnes 97, - D. C. Barnes, Comments on Plasma Phys. and Controlled Fusion 18, 71 (1997).

Vekshtein 90, - G. E. Vekshtein, Rev. Plasma Physics 15, Consuitants Bureau, NY (1990). Hirose 96, - A. Hirose, M. Elia, Phys. Rev. Lett. 76, 628 (1996).

Glasstone and Lovberg, 1960, - S. Glasstone, R. H. Lovberg, "Controlled Thermonuclear Fusion", D. Van Nostrand Co.,Inc., Princeton, NJ (1960).

Zel'dovich and Raizer, 1966, - Ya. B. Zel'dovich, Yu. P. Raizer, "Physics of Shock Waves and High-Temperature Hydrodynamic Phenomena", Academic Press, New York, NY (1966). LLNL, 1969, "Compilation of X-Ray Cross Sections", UCRL-50174-SEC 2-R1, U.S. Dept. of Commerce, National Technical Information Service NTIS, May 1969.

Prater 74, - R. Prater, Phys. Fluids 17, 193 (1974).

Hattori 1986, - F. Hattori, H. Takabe, K. Mima, Phys. Fluids 29, 1719 (1986).

Thayer 1987, - D. R. Thayer, P. H. Diamond, Phys. Fluids 30, 3724 (1987). 


\title{
APPENDIX D: PROPOSING TEAM BIOGRAPHIES
}

\author{
Roger J. Bartlett \\ Los Alamos National Laboratory
}

Roger Bartlett joined Los Alamos Scientific Laboratory in 1972 and is now a team leader in the Hydrodynamics and X-ray Physics Group, P-22. He received a BS and MS in Electrical Engineering and a Ph.D. in Physics from Iowa State University. A merit scholarship and a NFS fellowship supported his studies. While at Los Alamos he was the technical project leader for LANL's synchrotron radiation project at the National Synchrotron Light Source at Brookhaven National Laboratory and was the PI for five consecutive three year LDRD (Laboratory Directed Research and Development) projects in solid state and atomic physics. A common theme in his research has been the use of synchrotron radiation to probe the atomic and solid state properties of matter. He was one of the early users of synchrotron radiation as a tool for research having performed his first experiments on the Tantalus I storage ring (University of Wisconsin) in 1968. He has authored or co-authored over ninety refereed publications in these fields.

\section{James H. Degnan Air Force Weapons Laboratory}

Dr. James H. Degnan received his $\mathrm{PhD}$ in Physics in 1973 from the Dept of Physics and Astronomy, University of Pittsburgh, where he did his thesis work on experimental muclear reaction studies. This included charged particle, neutron, and gamma spectroscopy of reaction protucts from 12 to $18 \mathrm{MeV}$ light ion projectiles bombarding medium atomic number target nuclei. He worked at the Air Force Weapons Laboratory (AFWL), later known as the Weapons Laboratory (WL), later incorporated into the Phillips Laboratory (PL), and still later incorporated into the Air Force Research Laboratory, since 1973. There he designed, conducted, and directed research on gas puff and foil plasma implosions, puff gas coaxial plasma guns, inductive store - fuse opening switch and plasma flow switch development and application, subkilovolt X-ray diagnostic development (including fast high current bolometers and spectrum deconvolution codes), automated rezoners for Lagrangian MHD codes, diagnostics of neutron and gamma emission from $200 \mathrm{MeV}$ protons incident on thick targets, explosive magnetic flux compression generators, solid liner implosions (including cylindrical, conical, and quasi-spherical) and applications; compact toroid formation, compression, and acceleration; and compact, portable pulsed power for high impedance loads. He is presently a senior physicist (GS-15 = DR-IV) in the High Power Systems Branch of the Directed Energy Directorate of the Air Force Research Laboratory (AFRL/DEHP). Some recent, relevant publications include:

(1) "Compact Toroid Formation Experiments at the Weapons Laboratory", J.H.Degnan al, in ISPP-8 "Piero Caldirola", Physics of Alternative Magnetic Confinement Schemes, eds. S.Ortolani and E.Sindoni, Societa Italiana di Fisica, Bologna, Italy, 1991 (invited paper)

(2) "Compact Toroid formation, compression. and acceleration", J.H.Degnan et al, Phys.Fluids B5, 2938 (1993)

(3) “Electromagnetic implosion of a spherical liner", J.H.Degnan, et al, Phys.Rev.L.74, 98 (1995) 
(4) "Compression of compact toroids in conical-coaxial geometry", J.H.Degnan, et al, Fusion Technology 27, 107 (1995)

(5) "Multimegajoule electromagnetic implosion of shaped solid-density liners", J.H.Degnan, et al, Fusion Technology 27, 115 (1995)

(6) "Solid quasi-spherical shell liner implosions used to compress hot hydrogen working fluid", J.H.Degnan et al, presented at Megagauss-7 Conference, Sarov, Russia, Aug 96 (to be in Megagauss-7 Proceedings)

(7) "Formation, Compression, and Acceleration of Magnetized Plasmas", J.H.Degnan et al, in Current Trends in Fusion Research, Ed. E.Panarella, Plemum Press, p.179-195, 1997

\section{Carl Ekdahl \\ Los Alamos National Laboratory}

Carl Ekdahl earned his Ph. D. in Physics at the University of California, San Diego, in 1971. He spent two years as a Research Physicist at Scripps Institute of Oceanography measuring and interpreting the increase in atmospheric carbon dioxide resulting from mankind's use of fossil fuels. After spending the next two years at the Laboratory of Plasma Physics at Cornell University directing experiments to demonstrate heating of plasmas with intense relativistic electron beams, he joined Los Alamos National Laboratory in 1975 to carry out experiments in controlled thermonuclear fusion. In 1980 he left to join Mission Research Corporation in Albuquerque where he led experiments with atmospheric propagation of intense relativistic electron beams. He returned to Los Alamos in 1982 for a short while to lead further experiments to heat high-density plasma with electron beams, and to launch a high-power microwave source development program. In 1983 he joined the Sandia National Laboratories' to continue with beam propagation experiments and was promoted to Supervisor of the High-Energy Beam Physics Division the next year. He finally returned to Los Alamos in 1986 to design, execute, and analyze experiments using the radiation from underground nuclear weapon tests. As leader of a nuclear test diagnostics group he directed their transition into above ground experimental activities, including the first lab-to-lab experiments with VNIIEF. He is presently the program manager for High Energy Density Physics in Nuclear Weapon Technologies.

\section{John M. Finn \\ Los Alamos National Laboratory}

John Finn obtained his Ph. D. from the University of Maryland in 1974. His dissertation research was in the area of Lie transforms applied to particle motion in the magnetosphere and in mirror machines. This work was the basis for the Lie transform approach to particle dynamics in accelerators, developed into the code MARYLIE by Alex Dragt and co-workers. He did postdoctoral work at the Princeton University Plasma Physics Laboratory from 1974-76, where he worked on resistive instabilities and on destruction of magnetic flux surfaces as a model for tokamak disruptions. With Predhiman Kaw, he discovered and investigated the coalescence instability, which has been found to be a major aspect of nonlinear MHD. He worked during the period 1976-79 at Cornell University on kinetic and MHD instabilities in compact tori with an energetic ion component (ion rings). He and Ravi Sudan wrote a review paper on this subject which was published in Nuclear Fusion in 1982. He worked at the Naval Research Laboratory from 1979-82 in the area of compact tori and RFP's; specifically on ballooning and tilting modes in 
spheromaks and compact tori with an energetic ion component, and on resistive instabilities in RFP's and spheromaks, and their diamagnetic stabilization. He also worked on toroidal equilibria of electron beams in modified betatrons. He worked at the University of Maryland from 1982-93. During this time he worked in spheromak theory, specifically on spheromak formation in the MS device, on magnetic helicity and helicity injection, and on temperature gradient driven semi-collisional tearing modes in spheromaks and RFP's. He worked on convection and flow shear generation at the tokamak edge (H mode studies), discovering the linear instability responsible for generation of shear flow in the presence of convective vortices, and the manner in which this shear flow reduces the turbulence level. At this time he also worked in the magnetohydrodynamics of the solar corona and convection zone, specifically on MHD instabilities in 2D coronal arcades and 3D coronal loops, on the associated magnetic reconnection processes in 3D. He also worked on the fast dynamo problem, in which the flow was taken to have chaotic flow lines. His paper with Ed Ott was the first work to elaborate the relationship between the lagrangian chaotic properties of the flow and the intermittent aspects of the generated magnetic field. He has been at LANL since 1993. At LANL he has worked on linear and nonlinear studies of resistive wall instabilities and locking in tokamak geometry, and the application to tokamak disruptions. He discovered and investigated a new plasma instability driven by shear in the velocity parallel to the magnetic field, and discovered with $L$. Turner a new streaming instability in nonneutral beams with turning points. He has over 85 publications in refereed journals. In 1987 he was elected fellow of the American Physical Society.

\section{RECENT PUBLICATIONS}

1. "Resistive Wall Stabilization of Kink and Tearing Modes,"J. M. Finn, Phys. Plasmas 2, 198 (1995).

2. "Streaming Instabilities of a Nonneutral Plasma with Turning Points," L. Turner and J. M. Finn, Phys. Plasmas 2, 1378 (1995)..

3. "Stabilization of Ideal Plasma Resistive Wall Modes in Cylindrical Geometry: The Effect of Resistive Layers," J. M. Finn, Phys. Plasmas 2, 3782 (1995).

4. "New Parallel Velocity Shear Instability", J. M. Finn, Phys. Plasmas 2, 4400 (1995).

5. "Parallel Transport in Ideal Magnetohydrodynamics and Applications to Resistive Wall Modes", J. M. Finn and R. A. Gerwin, Phys. Plasmas 3, 2469 (1996).

6. "Mode Coupling Effects on Resistive Wall Instabilities", J. M. Finn and R. A. Gerwin, Phys. Plasmas 3, 2344 (1996).

7. "Time-Dependent Perturbation Theory for the Construction of Invariants of Hamiltonian Systems", H. R. Lewis, J. W. Bates, and J. M. Finn, Physics Letters A 215, 160 (1996).

8. "Magnetic Reconnection and the Topology of Interacting Flux Tubes", Y.-T. Lau and J. M. Finn, Phys. Plasmas 3, 3983 (1996).

9. "Orbital Resonances and Chaos in a Combined RF Trap", J. M. Finn, R. Nebel, A. Glasser, and H. R. Lewis, to appear in Phys. Plasmas (1997).

\section{Richard Gerwin \\ Los Alamos National Laboratory}

Dr. Richard Gerwin was awarded the D.Sc. degree with distinction in 1966, at the Technical University at Eindhoven, the Netherlands. His thesis was developed jointly under the auspices of this University and the Dutch government's Instituut voor Plasmafysica. It dealt with inertial effects in the diffusion of a plasma across a magnetic field, including the effects of $\mathrm{rf}$ fields. He worked in plasma physics at the Boeing Scientific Research Laboratories from 1959 to 1971, except for a two-year leave of absence for his thesis research in the Netherlands. He then worked at Los Alamos National Laboratory, beginning in 1971 until his retirement in 1995. Since his retirement, he has consulted at the Laboratory on plasma 
accelerators and on liner compression of fusion plasmas. He lead the Plasma Theory Group in alternate concepts research at Los Alamos, in the Controlled Thermonuclear Research Division (CTR), from 1979 through 1989; and Dr. Gerwin was elected a Fellow of the Amerian Physical Society in 1983. In 1979, he published a paper in Nuclear Fusion, with R. C. Malone, on compression of plasmas by compressible liners. Dr. Gerwin continues to consult at the Laboratory, and is also an adjunct professor of Nuclear Engineering at North Carolina State University, Raleigh, North Carolina.

\section{Lawrence Green \\ Program Manager, Fusion Programs \\ Westinghouse Science and Technology Center}

Dr. Green has has over ten years of fusion technology experience, including 6 years in the study of blanket design and shielding for fusion reactors. He also has over 20 years of experience in the area of fission reactor design, development, and safety. He is currently serving as Fusion Program Manager in the Energy Systems Engineering Department at the Westinghouse Science and Technology Center, where he is responsible for program development and engineering activities for all fusion-related activities. Current programs include the International Fusion Materials Irradiation Facility (IFMIF), ITER First Wall/Blanket/Shield, Plasma Facing Components, ITER Engineering Design Program, and ELISE Heavy Ion Beam Fusion Program.

As a Visiting Scientist at the Swiss Federal Institute of Technology (EPFL), Dr. Green was the Lead Experimental Scientist at the LOTUS facility, Lausanne, Switzerland, in a joint program involving Westinghouse, DOE and EPFL. This facility is dedicated to the experimental and theoretical study of fusion blankets and shielding. Dr. Green performed radiation transport studies and blanket and shield design at the Westinghouse Fusion Power Systems Department for magnetic and inertial confinement fusion systems. He participated in fusion reactor plant systems studies and conducted feasibility and design studies on the use of integral blanket neutronics experiments for data and code verification.

As a Visiting Professor in the Nuclear Engineering Dept., Ben Gurion University, Beer Sheva, Israel, Dr. Green performed studies on tight lattice, high-conversion water reactors and associated fuel cycles.

Dr. Green has authored or co-authored approximately 70 papers in fission and fusion-related technology.

\section{James H. Hammer \\ Lawrence Livermore National Laboratory}

James H. Hammer received his B.S.(Physics) from Arizona State University in1973 and his Ph.D.(Physics) at University of California-Berkeley in 1978. Began employment at Lawrence Livermore National Laboratory, 1979, Magnetic Fusion Energy, Theoretical Computations Group. Work has included examinations of various theoretical issues related to the Beta II compact torus (CT) experiment, a plasma model of the gun helicity injection and many studies (both numerical and analytical) of magnetohydrodynamic equilibrium and stability of several configurations. Served as program leader for compact torus acceleration experiment 1991-1994. Has made significant contributions to the invention and development of the CT accelerator, CT fueling and current dive for tokamaks, the CT pulsed X-ray source and the Fast Ignitor ICF concept. Holds a patent for a method of tapping electrical energy from the solar wind for space power and propulsion. Currently involved in radiation-magnetohydrodynamic modeling of dense, magnetized plasmas such as radiating z-pinches and laser-produced plasmas. 


\section{HONORS:}

Roy Lester Frank Memorial Award, 1978.

\section{Ronald C. Kirkpatrick \\ Los Alamos National Laboratory}

Dr. Kirkpatrick is currently a Non-proliferation and International Security Division staff member. He has degrees in Electrical Engineering (BS, 1959) and Physics (MS, 1963) from Texas A \& M University and in Astronomy (NASA Traineeship, $\mathrm{PhD}, 1969$ ) from the University of Texas. He has worked at Gulf States Utilities (Port Arthur, TX), NASA Ames Research Center (Mountain Veiw, CA), Southwest Research Institute (San Antonio, TX), Applied Research Lab (Austin, TX), and NASA Goddard Space Flight Center (NRC postdoc, Greenbelt, MD). He taught Physics and Astronomy at Texas A \& M University (1971-1972) before coming to Los Alamos in 1973.

At Los Alamos Dr. Kirkpatrick previously worked in the Thermonuclear Applications Group for 15 years and in the old Plasma Theory and Laser Fusion Group for 5 years. His chief expertise is in the areas of computational atomic physics, extreme non-LTE processes in astrophysical plasmas, fusion ignition physics, charged particle transport, and radiation transport. He originated the concept of an ignition critical profile, and long with Irv Lindemuth, he has advocated magnetized target fusion (MTF) for over a decade. From 1994 into 1995 he was the principle investigator for a Laboratory Directed Research and Development project for MTF theory and computation, and he participated in the first collaborative MTF related experiment (MAGO) with the Russian counterpart of Los Alamos. Dr. Kirkpatrick has numerous publications in the areas of atomic physics, astrophysics, and fusion physics, a few of which have numerous citations.

\section{Gerald F. Kiuttu \\ Air Force Research Laboratory}

Dr. Gerald F. Kiuttu received his BSE (Magna Cum Laude) in Engineering Science at Arizona State University in 1975. He received his MS in 1980 and his Ph.D. in 1986 in Nuclear Engineering (Plasma Physics) at the University of New Mexico. He worked at the Air Force Weapons Laboratory from 1975 to 1980 as a military officer, primarily on terawatt range soft X-ray and vacuum ultraviolet diagnostics. He was a research assistant in the Dept of Chemical and Nuclear Engineering at the University of New Mexico in 1980 to 1982, where his work included spatially resolved soft X-ray spectroscopy. As Senior Scientist at Mission Research Corp. in Albuquerque from 1982 to 1991 he worked on a variety of pulsed power innovations, applications and related diagnostics, including charged particle beams, high power microwaves, pulsed transformers, cloth fiber cathodes, hollow Z-pinches, plasma jet diagnostics, and more. Since 1991 he has been at the Air Force Research Laboratory (formerly Phillips Laboratory), where he is Pulsed Power Team Leader for the High Power Systems Branch. Here he has conducted and led research on compact toroids and explosive pulsed power systems. Compact toroid work included their use as fast switches and initiators of plasma focus-like discharges. He has worked on plasma injection, explosive flux compression generator design and modeling, and has fielded pulsed power diagnostics on large Russian explosive generators. He is co-inventor for U.S. Patent No 4918325, "Fast Risetime Pulse Power System," April 17, 1990 (AF Invention No 17.793). He is a member of the American Physical Society, the Institute of Electrical and Electronic Engineers, and the Sigma Xi Scientific Research Society. 


\section{Irvin R. (Irv) Lindemuth \\ Los Alamos National Laboratory}

Dr. Lindemuth is currently Project Leader for International Collaboration in the High Energy Density Physics Program at the Los Alamos National Laboratory, where his primary responsibility is to provide technical leadership for an historic scientific collaboration between Los Alamos and Los Alamos' Russian counterpart, the All-Russian Scientific Research Institute of Experimental Physics (VNIIEF) at Sarov (Arzamas-16). Prior to joining Los Alamos in 1978, he was a technical staff member in A-Division at the Lawrence Livermore National Laboratory where he was involved in fusion research. Dr. Lindemuth received his B.S. degree in Electrical Engineering from Lehigh University in 1965 and his M.S. and Ph.D. degrees in Engineering-Applied Science from the University of California, Davis/Livermore in 1967 and 1971, respectively. His areas of expertise include thermonuclear fusion and advanced numerical methods for the computer simulation of fusion plasmas and related pulsed power technology. He has published numerous papers in refereed journals and proceedings of major international conferences. He has been involved in a wide range of fusion and high energy density physics programs spanning essentially all of the ten orders of magnitude in density and time space from magnetic fusion energy plasmas to inertial confinement fusion plasmas. An internationally recognized pioneer in the application of implicit, nonsplit computational methods to magnetohydrodynamics, he has achieved widespread recognition for his large-scale numerical simulations of a variety of fusion and other high-density plasma systems. In addition to his accomplishments in modeling high temperature plasmas, he has formulated a variety of novel pulsed power computer codes that have led to important advances in laboratory programs. His codes have stimulated the development of several types of fast opening switches, and he has designed opening switch experiments, which set US records for transfer of explosively generated electrical energy. He is a US pioneer on magnetized target fusion (MTF) and performed the first comprehensive survey of the parameter space in which MTF was likely to work. Even before the collapse of the Soviet Union, he recognized that the Soviets had developed advanced technology in the areas of ultrahigh magnetic fields and ultrahigh energy electrical pulse generation which significantly exceeded US capabilities and which were motivated by the Soviet MTF program known as MAGO. Dr. Lindemuth played an essential role in establishing the collaboration with VNIIEF, which provides US access to Russian advances in MTF and pulsed power technology. In 1992, he was the recipient of a Los Alamos Distinguished Performance Award for his work in the formative stages of the LANL/VNIIEF collaboration. His relationship with Russian scientists will ensure that the US takes full advantage of Russian advances relevant to MTF and his computational expertise will ensure that the US MTF program has a strong synergism between experiment, theory, and detailed, multidimensional computational modeling.

\section{Richard D. Milroy \\ Redmond Plasma Physics Laboratory. University of Washington}

Since January of this year, Dr. Milroy is a "Principal Research Scientist" at the University of Washington's Redmond Plasma Physics laboratory. He is primarily responsible for developing and applying numerical models in support of the experimental fusion related plasma physics research program at this laboratory.

From December of 1992 through December of 1997, Dr. Milroy was "Director of Software Development" at MCM Enterprise Ltd., in Bellevue WA. During that time he led a small team to develop 
commercial software to analyze data from hydroelectric generator instrumentation. This work included the recent development of a complete data acquisition and analysis package using an Expert System for data interpretation and analysis. He was also responsible for the development of Allen-Bradley's MessageBuilder software product. This commercial product is used to configure a line of AllenBradley's industrial control display terminals.

Prior to that Dr. Milroy spent 15 years (1978-1992) at STI Optronics in Bellevue WA, where he became a nationally recognized expert in the field of computational plasma physics. He has worked extensively in the areas of Field Reversed Configuration (FRC) formation, stability, and transport. This work has involved the development of and application of several numerical models including dynamic two and three-dimensional MHD computer codes to study the formation and stability of FRCs. In addition, he has developed numerical models in support of other areas of research at STI. These include the energy exchanger FLOW code to evaluate the parametric performance of the STI energy exchanger, a hydrodyanamic model of laser flow loops, a Monte-Carlo simulation of high energy (relativistic) electrons with a gas in an arbitrary electro-magnetic field, and a integrated model including hydrodynamics, discharge physics and gas kinetics for simulating high power transverse flow $\mathrm{CO}_{2}$ lasers.

Dr. Milroy has authored or co-authored over twenty refereed publications.

\section{Raiph W. Moir \\ Lawrence Livermore National Laboratory}

Education

B.S. 1962--Engineering Physics, University of California, Berkeley

Sc.D. 1967--Nuclear Engineering, MTT, Cambridge, Mass.

Professional Associations, Societies and Honors

Registered Professional Nuclear Engineer in the State of California, Registration number NU782.

American Physical Society, Fellow 1981, Plasma Physics Division

American Nuclear Society, Fellow 1989, Fusion Energy Division,

American Association for the Advancement of Science

Publications

1. R. W. Moir and R. F. Post, "Yin-Yang Minimum--B Magnetic Field Coil", Nuclear Fusion, 9, 253 (1969).

2. R. W. Moir and W. L. Barr, "Venetian-Blind Direct Energy Converter for Fusion Reactors", Nuclear Fusion, 13, 35-45 (1973).

3. R. W. Moir, "The Fusion-Fission Fuel Factory, Chapter 15, p. 411-451, in Fusion, Vol. 1 Part B, edited by E. Teller, Academic Press, New York (1981).

4. R. W. Moir, al., "Study of a Magnetic Fusion Production Reactor", A series of eight articles on tritium production. J. Fusion Energy, 5, 255-331 (1986) and 6, 3-88 (1987).

5. R. W. Moir, "Pacer Revisited", Fusion Technology 151114 (1989).

6. B. G. Logan, R. W. Moir, M. Tabak, R. L. Bieri, J. H. Hammer, C. W. Hartman, M. A. Hoffman, R. L. Leber, R. W. Petzold, M. T. Tobin, "Compact Torus Driven Inertial Confinement Fusion Powe Plant HYLIFE-CT," UCRL-ID-106403 (1991). SRD

7. A. Szoke and R. W. Moir, "A Practical Route to Fusion Power," Technology Review, 94 p 20- 27 (July 1991). 
8. R. W. Moir at al., "Inertial Fusion Energy Power Plant Design Using the Compact Torus Accelerator: HYLIFE-CT ," Fusion Technology 211492 (1992).

9. R. W. Moir, R. L. Bieri, X. M. Chen, T. J. Dolan, M. A. Hoffman, P. A. House, R. L. Leber, J. D. Lee, Y. T. Lee, J. C. Liu, G. R. Longhurst, W. R. Meier, P. F. Peterson, R. W. Petzoldt, V. E. Schrock, M. T. Tobin, W. H. Williams, "HYLIFE-II: A Molten Salt Inertial Fusion Energy Power Plant Design-Final Report," Fusion Technology 25 (1994) 5-25.

10. S. Sahin, R. W. Moir, and S. Unalan, "Neutronic Investigation of a Power Plant Using Peaceful Nuclear Explosives,"Fusion Technology 26 (1994) 1311-1325.

11. R. W. Moir, "Liquid First Walls for Magnetic Fusion Energy Configurations," Nuclear Fusion 37 (1997) 557-566.

\section{Ronald W. Moses, Jr. Los Alamos National Laboratory}

Ron Moses is a Technical Staff Member in the Fluid Dynamics Group (T-3) at Los Alamos National Laboratory. Dr. Moses received his Ph.D. in Physics from the University of Wisconsin, Madison in 1968, specializing in the physics of electron imaging. After leaving Wisconsin, he was an NSF Postdoctoral Fellow for one year at the Cavendish Laboratory, Cambridge University, England. Over the next several years he held research and teaching positions in the Institut fur theoretische Physik of the Technische Hochschule Darmstadt, Germany; the University of Chicago; and the University of Wisconsin, Madison. Ron joined Controlled Thermonuclear Research Division.(CTR) at Los Alamos National Laboratory in 1976. He is an author of over 100 scientific papers and reports in the subject areas of plasma physics, particle optics, wave optics, accelerator physics, and superconducting energy storage. While in CTR Division, Ron was the co-originator of the kinetic dynamo theory of Reversed-Field Pinch sustainment. He was also leader of a three person research team in the explanation of a collisionless magnetic reconnection and plasma heating mechanism. From 1990 to the present Ron has worked in the fields of pulsed power, magnetized target fusion (MTF), ground penetrating radar, atmospheric infrasound, and fluid turbulence. Ron has had a long-standing interest in MTF; he led the effort referenced as: $R$. W. Moses, R. A. Krakowski and R. L. Miller, "A Conceptual Design of the Fast-Liner Reactor (FLR) for Fusion Power," (February, 1979) LA-7686-MS. This year-long MTF project addressed issues ranging from basic plasma physics to the engineering of blast containment. Ron has contributed to the plasma physics and systems modeling of LANL's recent efforts in MTF. He brings to this project a broad range of experience in physics and engineering as well as expertise in MTF.

\section{Dr. Paul B. Parks \\ General Atomics}

Dr. Parks is a plasma physicist, with a strong and diversified interdisciplinary background in classical physics and applied science. He has extensive experience in a number of diverse theoretical and applied areas connected with magnetic and inertial fusion research. He has published 57 papers in the refereed Physical Journals and published a book on pellet ablation in plasmas. Highlights of his scientific accomplishments and creativity are given below in chronological order:

Dr. Parks is especially recognized for his pioneering work on pellet/plasma interaction theory, and predictions of his widely accepted pellet ablation model agree well with measured pellet ablation rates in tokamak plasmas. 
He developed a novel plasma impurity control concept using radio frequency waves as a momentum source.

Dr. Parks performed fast wave ICRH antenna coupling calculations and developed the theory of Faraday shields for application to cavity launchers. He holds (with others) a U. S. Patent for a novel ICRH cavity launcher, which was built and installed on the DIII-D tokamak

Dr. Parks made a theory of current drive in tokamak plasmas which involved combining ECRH with an induced local gradient in the toroidal magnetic field.

For five years, Dr. Parks provided theoretical support for the GA/Nagoya mirror fusion program involving the RFC-XX double cusp device, and interacted with LLNL on related mirror problems. His most notable contribution was introducing an innovative method of stabilizing mirror plasmas using radio-frequency pondermotive forces. The method was successfully confirmed by experiments on the Phaectrus device at $U$. of Wisconsin.

He has conducted SDI related research in several applied areas involving high-power microwave generation, MHD and gas-dynamic phenomena in electromagnetic railgun launchers, exploding foil fuses, hypersonic plasma wind tunnels, and other related devices. He developed a theoretical model of a variable cross section axial-flow Z-pinch for hyper velocity projectile acceleration.

With Dr. R. Fisher, he started a DOE-funded program in collaboration with PPPL using high-speed low$Z$ refractory pellets as a plasma diagnostic tool for measuring the distribution function of confined alpha particles During the past seven years he was in charge of the modeling effort for the Pellet Charge Exchange Diagnostic project, which recently led to the first measurements of fusion born fast alpha particles inside the Princeton TFTR tokamak

During the spring of 1991 and summer of 1995 he was a visiting scientist at the Max-Planck Institute for Plasma Physics, Garching, Germany, where he worked on several pellet ablation problems and divertor surface ablation by high-heat fluxes induced during plasma distuptions. One outstanding issue was the source of pellet ablation oscillations. He developed a theory explaining the oscillations which invoked $\mathrm{E} \times \mathrm{B}$ rotation drive as the cause of a magnetized Rayleigh-Taylor instability.

Currently Dr. Parks has received funding from ITER to work on a new concept proposed by $M$. Rosenbluth involving the injection of cryogenic liquid jets in the plasma to mitigate plasma disruptions. Models for penetration, jet breakup time, and stability, are being developed in collaboration with ITER. He is also leading the effort for a proof-of-principle experiment on DII-D.

\section{Robert E. Reinovsky \\ Los Alamos National Laboratory}

Robert E. Reinovsky received his ME degree in 1971 and his $\mathrm{PhD}$ in 1973 from Rensselaet Polytechnic Institute in the Electrophysics Department. From 1974-1986 he worked at the AF Weapons Laboratory (now the AF Phillips Laboratory) in plasma and pulse power physics, where his principle interests were high density plasma implosions, radiation processes, plasma diagnostics and pulse power physics. He was responsible for a developing and building four generations of the world-class SHIVA family of high curremt, low impedence pulse power systems. Techniques in ultra high current, high explosive pulse power that had develped in Los Alamos starting in the 1950's caught his imagination. He joined Los Alamos National Laboratory in 1986 to continue work applying these techniques to ultra-high current 
plasma systems for applications to High Energy Density Physics. He led the explosive pulse group from 1990 to 1993 and then joined the HEDP program as project leader for! the Athena pulse power project and then as Project Leader/Chief Scientist for the HEDP program.

\section{Edward L. Ruden \\ Air Force Research Laboratory}

Dr. Edward L. Ruden, Ph.D. Physics, UC Irvine, 1988. Since Dr. Ruden received his degree, he has performed research in the area of high energy density states of matter for the Air Force Research Laboratory's High Power Systems Branch and its predecessors. He has developed several interferometric systems for the diagnosis of plasmas using coherent radiation sources across a broad spectrum: millimeter wave, far IR, visible, and UV. His latest effort in this area has been to push the spectrum into the soft Xray regime. Specific plasma geometries that Dr. Ruden has studied (interferometrically and by other means) include gas-puff Z-pinches, capillary discharge Z-pinches, longitudinally accelerated compact toroids (spheromaks), and high pressure plasmas compressed by electromagnetically imploded solid liners. In addition, he has significant experience in solid material elastic-plastic flow physics. Experimental accomplishments in this area include the development of fast closing valves and blast shutters using implosions driven electromagnetically ( $\theta$-pinched) or by chemical explosives, and a cryogenic frozen fiber extrusion system. Dr. Ruden has also performed theoretical work in the high strain rate elastic-plastic flow of metals which has lead to improvements in the treatment of shock and plastic work heating, and the plastic and Rayleigh-Taylor instabilities of strongly accelerated solids. Representative authorships and coauthorships include:

- J.H. Degnan, et. al., "Formation, Compression, and Acceleration of Magnetized Plasmas", Current Trends in International Fusion Research, E. Panarella, ed., 179-196, Plenum Press, New York, NY. 1997

- F.J. Wessel, N. Rostoker, H.U. Rahman, P. Ney, E.L. Ruden, "Thermonuclear Fusion in a Staged Pinch", Current Trends in International Fusion Research, E. Panarella, ed., 333-345, Plenum Press, New York, NY, 1997

- E.L. Ruden and D.E. Bell, "Rayleigh-Taylor stability criteria for elastic-plastic solid plates and shells", J.Appl.Phys., July 1, 1997 issue (date tentative).

- H.U. Rahman, E.L. Ruden, K.D. Strohmaier, F.J. Wessel, and G. Yur, “Closed cycle cryogenic fiber extrusion system", Rev. Sci. Instrum. 67, 1996, 3533-3536.

- J.H. Degnan, et.al., "Electromagnetic Implosion of Spherical Linex", Phys. Rev. Lett., 74, 98-101 (1995).

- J.H. Degnan, et.al., "Compression of Compact Toroids in Conical-Coaxial Geometry", Fusion Technology, 27, 107-113 (1995).

- E.L. Ruden, J.H. Degnan, T.W. Hussey, M.C. Scott, J.D. Graham, and S.K. Coffey, "Time resolved mass flow measurements for a fast gas delivery system", Rev. Sci. Instrum. 64, 1993, 1740-1742.

- E.L. Ruden, B.W. Mullins, M.E. Dearborn, and S.K. Coffey, "Interferometry on the compact toroid formation experiments at Phillips Laboratory”, Phys. Fluids B, 4, 1800-1805, 1992.

\section{Dmitri D. Ryutov \\ Lawrence Livermore National Laboratory}

Dmitri Ryutov obtained his Ph.D. in Plasma Theory from the Kurchatov Institute, Moscow, Russia, in 1965. Since July 1994, he has been working as a physicist in the Energy Program at Lawrence Livermore National Laboratory. Previously he was Deputy Director at the Budker Institute of Nuclear Physics, Novosibirsk, Russia, and a junior scientist at the Kurchatov Institute, Moscow, Russia. Dmitri is 
generally interested in plasma physics and its applications, environmental aspects of energy proctuction, advanced dynamics, and space plasmas. He received the I. V. Kurchatov Fellowship in 1960-62, gracuated Summa Cum Laude, Moscow Institute of Physics and Technology in 1962, and is Academicain, Academy of Sciences of Russia. Dmitri also is a member of the American Physical Society, the American Association for the Advancement of Science, and the European Physical Society.

RECENT PUBLICATIONS:

[1] "Environmental Aspects of Fusion Energy", Plasma Physics and Controlled Fusion, v. 34, p.1805 (1992)

[2] "Velocity shear effects in the problem of the electron temperature gradient instability incuced by conducting end walls". Physica Scripta, v.50, p.153 (1994) - in co-authorship with K.Lotov and J.Weiland

[3] "Charge and current neutralization in the formation of ion rings in a background plasma," Physics of Plasmas, v. 1, p.3383 (1994) - in co-authorship with B.Oliver and R.Sudan.

[4] "Mirror fusion research: update and prospects," Comments on Plasma Physics and Controlled Fusion, v 16, p.375 (1995) - in co-authorship with R.F.Post.

[5] "Spinning laser targets," Comments on Plasma Physics and Controlled Fusion, v 17. p.1 (1995) - in co-authorship with D.Baldwin.

[6] "Kinetic theory analysis of sheaths and shocks," Contrib.Plasma Phys., v.36. 207 (1996).

[7] "Rayleigh-Taylor Instability in a Finely Structured Medium," Physics of Plasmas, v.3, 4336 (1996).

[8] "Submegajoule liner implosion of a closed field line configuration," Fusion Technology, v. 30.310 (1996) - in co-authorship with P. Drake, J. Hammer, C. Hartman and J. Perkins.

[9] "Plasma convection induced by toroidal asymmetries of the divertor plates and gas puffing," Nuclear Fusion, Vol. 37, 621 (1997) - in co-authorship with R. Cohen.

[10] "The role of finite photon mass in magnetohydrodynamics of space plasmas," Plasma Physics and Controlled Fusion, v. 39 (1997).

\section{Kurt F. Schoenberg \\ Plasma Physics Group Leader \\ Los Alamos National Laboratory}

Dr. Kurt F. Schoenberg leads the 100-member plasma physics group (P-24) at the Los Alamos National Laboratory. Here, he directs experimental Laboratory research in Inertial Confinement Fusion, Magnetic Fusion, laser-based weapons physics, and plasma processing for industry, defense and the environment. His recent research interests and activities have focused on developing magnetically-nozzled coaxialplasma-accelerators for advanced incustrial manufacturing and advanced space propulsion. This research effort has developed a commercial manufacturing technology for microelectronics with the 3M Company.

From 1979 to 1991, Kurt was principally involved in the experimental and theoretical investigation of magnetically confined plasmas for controlled thermonuclear fusion, where he lead the ZT-40M experiment and research group. Here, his research interests included plasma dynamos, plasma sustainment by magnetic helicity injection (oscillating field current drive), RFP plasma transport by magnetic and electrostatic turbulence, similarities in edge plasma transport between tokamaks, stellarators and RFPs, and turbulence-driven anomalous ion heating. He was also a major contributor to the design and construction of the ZT-40M, ZT-P and ZTH experiments.

Over the past several years, Kurt has welcomed participation in the progressive evolution of the US Fusion program by serving on several key committees, including the New Initiatives Task Force (1992), TPX Physics Advisory Committee (1993 - 1996), TPX National Stering Committee (1995), FEAC SiCom Panel on Alternates (1996), and the FESAC ITER Panel 2 (1997). However, it is his recently 
acquired interest in inertial fusion, coupled with the re-awaking of the US MFE program to the need of better-cheaper-faster approaches to fusion, that motivate his present interest in pursuing MTF as a lowcost development path to fusion energy.

\section{Peter T. Sheehey \\ Los Alamos National Laboratory}

Peter T. Sheehey is a technical staff member in the Nuclear and Hydrodynamics Applications group (X$\mathrm{NH}$ ), and has been at LANL since 1991. As a computational plasma/fluid physicist, he has done multidimensional modeling of MTF target plasmas, such as fiber Z-pinches and MAGO, MTF-suitable highenergy liner systems, and has participated in joint U.S.-Russian MTF experiments.

Jack Shlachter

\section{Los Alamos National Laboratory}

Jack Shlachter (Deputy Group Leader, P-22 Hydrodynamic and X-ray Physics) has been at Los Alamos National Laboratory since 1979. He holds a B.S. with Honors in Physics from CalTech and a Ph.D. in Physics from University of California at San Diego. He has worked extensively in the areas of magnetic confinement fusion and high energy density physics and contributed to the development of the highdensity z-pinch. His experimental responsibilities have included the design, implementation, and analysis of interferometric and $\mathrm{x}$-ray data on magnetized plasmas.

Recently, he served as the project physicist for the Atlas pulsed power facility. He has authored or coauthored over a dozen refereed publications.

\section{Richard E. Siemon \\ Fusion Energy Program Manager \\ Los Alamos National Laboratory}

Currently, Dick Siemon is the Program Manager for LANL's \$3.5-million Office of Fusion Energy research program. Los Alamos research includes ITER-relevant tritium and beryllium technology; experimental collaborations, involving plasma diagnostics on large tokamak facilities at other laboratories; theory, aimed at improved the understanding of tokamak stability limits; and an innovative concept called the Penning Fusion Experiment. Dick serves on ISCUS, the US Steering Committee for ITER. He was appointed by previous Secretaries of Energy to serve on the Magnetic Fusion Advisory Committee (1986-1990) and the Fusion Energy Advisory Committee (1990-1994). From 1978 to 1988 Dick was the Group Leader of a 30-person group that carried out extensive studies of the Field Reversed Configuration. Before that he worked on numerous non-tokamak concepts including high-beta stellarators, linear theta pinches, and multiple magnetic mirrors. A Fellow of the American Physical Society, his experimental research included advances in plasma diagnostics, such as holographic interferometry, and innovations for Thomson scattering. Prior to termination of US alternative concept research in 1990, Dick was in the Los Alamos CTR Division office as Fusion Energy Program Manager, where he helped supervise construction of the \$75-million Compact Physics Research Facility, which was intended to study, as a first step, the ZT-H Reversed Field Pinch. In 1995, Sig Hecker, Laboratory Director, appointed Dick as one of six Los Alamos Industrial Fellows, a pilot program to improve Laboratory outreach to US Industry. Dick spent one year at Dow Chemical headquarters in Midland, Michigan. Following that he has served approximately half time as the laboratory-wide coordinator of the 
ongoing Industrial Fellows Program, and he continues to work with Dow Chemical as a consultant on management issues surrounding the use of external $R \& D$.

\section{Dr. Y. C. Francis Thio \\ Massey University, Auckland, New Zealand}

A versatile experimental, theoretical and computational physicist, Dr. Francis Thio is an international with a track record in a number of high energy density physics areas including fusion energy, pulsed power, electromagnetic launch, impact physics and warheads, as well as areas in geophysics and biophysics. He has authored over 50 technical publications in these areas combined and hold four patents. He was among the first researchers to set the record of launching a gram-size projectile reproducibly in the laboratory to orbital velocity $(8.2 \mathrm{~km} / \mathrm{s})$ using a high density plasma driven by submegagauss magnetic field in 1986.

He has made fundamental contributions to the analytical and numerical solution of equations of mathematical physics in the area of electromagnetic fields, geophysics and plasma physics. He has made computations of the transport properties and the equation of state of dense and strongly coupled plasmas $\left(10^{24}\right.$ to $10^{28} \mathrm{~m}^{-3}$, with non-ideal parameter $\gamma>0.5$ ), and the simulations of plasmas using finitedifference MHD codes, with implicit methods and flux-corrected-transport (FCT) algorithm. In solid mechanics, he has performed critical mechanical design of high power electromagnetic equipment using finite-element stress analysis. In instrumentation, he has designed, developed and/or applied magnetic probes, pulsed current probes, electrical (plasma) probes, voitage probes, $x$-ray probes, and emission spectroscopy, including extensive experience in optoisolation, optoelectronics, digital data acquisition, computer control of experiments, high-speed pulsed electronics, computer data manipulation and analysis.

He has been a Principal Investigator and Program Manager of research programs and grants totaling more than $\$ 40 \mathrm{M}$ over a period of 15 years from 1982 to 1996 in the US prior to an appointment in New Zealand. He has held assignments as Scientific Advisor and/or Consultant to Los Alamos National Laboratory, Strategic Defense Initiative Office (Pentagon), Defense Nuclear Agency, Naval Air Engineering Center (Lakehurst, NJ), David Taylor Research Center(Annapolis, MD), and SPARTA, Inc.

Employment record:

1996-Present: Section Leader, Mathematics, Massey University, Auckland, New Zealand.

1991-1996: Associate Professor of Physics, University of Miami, Coral Gables, FL.

1988-1990: Scientific Associate, Physics Division, Los Alamos National Laboratory.

1986- 1988: Senior Research Physicist, GT-Devices (subsidiary of General Dynamics), VA.

1982 - 1986: Senior Research Scientist, Westinghouse R\&D Center, Pittsburgh, PA.

1978 - 1982: Research Scientist, Aeronautical and Maritime Research Laboratory, a National Laboratory of the Defence Science and Technology Organization, Australia.

1977 - 1978: Postdoctoral officer, Computer Science, Monash University, Melbourne, Australia.

Visiting Appointments:

1997, 1998: Consultant, Los Alamos National Laboratory.

1994-1995: Senior Fellow, Physics Division, Nanyang Technological University, Singapore. 
1994, 1995: Summer Faculty, High Energy Plasmas Division, Phillips Laboratory, Kirtland AFB, NM. 1989-1990: Visiting Associate Professor, College of Engineering and Physics, U. of nlinois at UrbanChampaign, Illinois.

Distinctions/Honours:

1996-Present Associate Editor of Physics Essays.

1994-Present Member of the International Steering Committee, Symposium on Current Trends in International Fusion Research.

1986 Westinghouse Signature Award of Excellence for outstanding contributions in plasma science.

1982 The Australian Defense Science and Technology Organization Achievement Honour, Inaugural Award for outstanding contributions in electric propulsion.

1974-1977 The Australian Commonwealth Postgraduate Research Award.

1969-1973 British Commonwealth Colombo Plan Undergraduate Scholarship.

Peter J. Turchi

Air Force Research Laboratory.

Education: BSE (1967), MA (1969), PhD (1970), Aerospace and Mechanical Sciences, Princeton University, Princeton, NJ.

Career highlights: Plasma Physicist, Air Force Weapons Laboratory (1970-72); Chief, Plasma Technology Branch, Naval Research Laboratory (1977-80); Director, Washington Research Laboratory, R\&D Associates, Inc. (1981-89); Professor, Aerospace Engineering, Applied Mechanics and Aviation Department, The Ohio State University (1989- present); Senior Research Scientist, IPA, Phillips Laboratory (1990-present); Visiting Chief Scientist for Advanced Weapons and Survivability, Phillips Laboratory (1996-1997).

Research area: high energy density plasma and pulsed power systems, including imploding liner systems for controlled fusion at megagauss magnetic field levels.

Related patents (P.J.Turchi):

1. "EM Implosion X-ray Source", with W.L. Baker (1973).

2. "Stabilized Liner Implosion System", with D.J. Jenkins (1979).

Other relevant professional activity (P.J.Turchi):

DoE Panels on Alternative Fusion Concepts (1977 - 1978)

DoE Review Panel on Field Reversed-Mirror Plasma Generation (1978)

Editor, Megagauss Physics and Technology, Plenum Press, NY (1980).

Board of Directors, Megagauss Institute (1979 - present)

- Chairman (1979 - 1991)

International Advisory Committee for Megagauss Conferences (1978 - present) 


\section{Tuszewski \\ Los Alamos National Laboratory}

Michel Tuszewski is a plasma physicist at LANL since 1980. He became an Applied Plasma Team Leader and a Laboratory Fellow in 1997. He received an Engineering and Physics M.S. from the Ecole Centrale de Paris, France, in 1971. He came to UC Berkeley in 1972 with Fullbright and French Government grants. He received MS and PhD in Nuclear Enginnering from UCB in 1973 and 1976, respectively. He was a research associate at Cornell University (1977-1978), a lecturer at UCB (19781979), and a visiting scientist in France (1986) and Japan (1990).

Tuszewski combines experimental and theoretical plasma physics skills. He has studied magnetic fusion since 1973, including Multiple Mirrors, Relativistic Electron and Ion Beams, Compact Torii, and Tokamaks. He is an acknowledged expert in the area of Field Reversed Configurations. Recently, he has developed research interests in inductive discharges for materials processing and in Magnetized Target Fusion.

He has published over 100 refereed journal articles in various fields of plasma physics, including an FRC Review Article in Nuclear Fusion. He has written one book chapter, signed 4 CRADAS, and filed 5 patents in plasma processing. He is a reviewer for many scientific journals and DOE panels. He joined the American Physical Society in 1975, became an APS Fellow in 1989, and an APS life-member in 1990.

\section{Glen A. Wurden \\ Los Alamos National Laboratory}

Glen A. Wurden, presently a staff physicist and Team Leader of the MFE Section in the P-24 Plasma Physics Group at Los Alamos, was born on Sept. 9, 1955 in Anchorage, Alaska. He attended public schools in western Washington, and went to the University of Washington on a National Merit Scholarship. There he earned three simultaneous B. S. degrees, in Physics, Mathematics, and Chemistry, summa cum laude (1977), graduating with the highest class honors as President's Medalist. He was awarded a National Science Foundation Graduate Fellowship, and chose Princeton University's department of Astrophysical Sciences to specialize in Plasma Physics for his M.S. (1979) and Ph.D. (1982) Degrees. He is a member of Phi Beta Kappa, IEEE, and the American Physical Society.

He spent the summer of 1979 as a staff physicist working on x-ray and alpha particle imaging of inertial fusion targets on the Shiva laser at Lawrence Livermore Laboratory in California. Upon finishing his Ph.D. degree (" $\mathrm{CO}_{2}$ Laser Scattering on Radio-Frequency Waves in the Advanced Concepts Torus") at Princeton, he obtained a position at Los Alamos National Laboratory in New Mexico as a J. R. Oppenheimer Postdoctoral Fellow in the CTR-8 plasma diagnostics group, and after two years, a permanent staff position in the CTR-2 reversed field pinch experimental group. In August 1988 he moved to Germany for 16-months as a DOE Exchange scientist, working in the Max Planck Institute for Plasma Physics on the ASDEX tokamak, in Garching near Munich. After his return to Los Alamos at the end of 1989 , he worked on the ZTH construction project (FIR interferometer, soft x-ray arrays, pellet injection) before taking a leave of absence to the $U$ of Washington as an Acting Associate Professor of Nulcear Engineering in August 1990. He returned to the P-1 group (High Energy Density Physics, now P-24 Plasma Physics) at LANL in April 1992, and is presently working on diagnostic collaborations at TFTR (Princeton), JT-60U (Naka, Japan), Alcator C-Mod (MIT), and HBT-EP (Columbia University). He is a 
member of the ATLAS Design Team, principally involved in diagnostic, target chamber, and MTF issues.

His research interests include a wide range of plasma diagnostic techniques, and their application to better understanding complex processes in hot fusion plasmas. He has particular research interests in farinfrared lasers, laser scattering, bolometry, fast pellet injection, fast $\mathrm{x}$-ray and visible light imaging, neutron measurements, and concept improvement in fusion devices.

G. A. Wurden, B. J. Peterson, and Shigeru Sudo, "Design of an imaging bolometer system for the large helical device", Rev. Sci. Instru., 68(1), 766-769 (1997).

G. A. Wurden, R. E. Chrien, C. W. Barnes and W. C. Sailor, "Scintillating-fiber $14 \mathrm{MeV}$ neutron detector on TFTR during DT operation", Rev. Sci. Instrum. 66(1), $901-903$ (1995).

G. A. Wurden, M. Sasao, D. Mansfield, "Alpha particle detection via Helium spectroscopy in Lithium pellet cloud", LA-UR-94-667, Alpha Particle workshop, Princeton, NJ, March 2-4, 1994.

G. A. Wurden, S. Jardin, D. Monticello, H. Neilson, "Disruption control strategies for TPX", LA-UR-932367, US-Japan Workshop on Steady-State Tokamaks, Kyushu, Jun 29-July 2, 1993.

G. A. Wurden, R. J. Maçueda, et al. "Initial Experimental results from the LSX field reversed configuration", 1991 EPS Conference, Berlin, Vol. 15C, part II pg 301-303.

G. A. Wurden, P. G. Weber, R. G. Watt, et al, "Pellet refueling of the ZT-40M reversed field pinch", Nuclear Fusion 27(5), 857-862 (1987).

G. A. Wurden, "Soft x-ray array results on the ZT-40M RFP", Phys. Fluids, 27(3), $551-554$ (1984).

G. A. Wurden, "Ion temperature measurement via laser scattering on ion Bernstein waves", Phys Rev A, 26(4), 2297 (1982).

\section{Frederick J. Wysocki \\ Los Alamos National Laboratory}

Frederick J. Wysocki is an experimental physicist specializing in plasma physics. He has worked on the spheromak magnetized-plasma configuration at both the Princeton Plasma Physics Laboratory (PPPL) and at the Los Alamos National Laboratory (LANL). The work at PPPL was in the context of controlled thermonuclear fusion, while the spheromak work at LANL was in the context of the defense program and a novel approach to achieving hypervelocity. During his time at AT\&T Bell Laboratories Fred built a positron trap for the purposes of producing a single-component positron plasma. Before leaving Bell, trapping of positrons was successfully demonstrated. Within P-Division at LANL, Fred has worked on several high particle and energy density plasma configurations, including the high-density-Z-pinch (HDZP) ZEBRA project, foil-implosion z-pinches and plasma-flow vacuum-opening-switch development in the Trailmaster, Athena, and HEDP programs, and the dense-plasma-focus (DPF) configuration on the Colt facility. Recently, Fred is working on developing a target plasma for magnetized target fusion using the Colt facility and funded by LANL internal LDRD funds. Fred's expertise includes diagnostics (magnetic probes, laser interferometry, Thomson scattering, Lagmuir probes), data analysis and interpretation, plasma modeling including running 2-dimensional magnetohydro-dynamic simulation codes, data acquisition, pulsed-power technology. facility operation, and the design and execution of plasma experiments. 